TRANSACTIONS OF THE

AMERICAN MATHEMATICAL SOCIETY

Volume 365, Number 9, September 2013, Pages 4633-4677

S 0002-9947(2013)05885-0

Article electronically published on April 9, 2013

\title{
WEAK SOLUTIONS TO THE HEAT FLOW FOR SURFACES OF PRESCRIBED MEAN CURVATURE
}

\author{
VERENA BÖGELEIN, FRANK DUZAAR, AND CHRISTOPH SCHEVEN
}

ABSTRACT. In this paper we establish the existence of global weak solutions to the heat flow for surfaces of prescribed mean curvature, i.e. the existence for the Cauchy-Dirichlet problem to parabolic systems of the type

$$
\left\{\begin{array}{c}
\partial_{t} u-\Delta u=-2(H \circ u) D_{1} u \times D_{2} u \quad \text { in } B \times(0, \infty), \\
u=u_{o} \text { on } \partial_{\text {par }}(B \times(0, \infty)),
\end{array}\right.
$$

where $H: \mathbb{R}^{3} \rightarrow R$ is a bounded continuous function satisfying an isoperimetric condition, $B$ is the unit ball in $\mathbb{R}^{2}$ and $u: B \times(0, \infty) \rightarrow \mathbb{R}^{3}$. As one of the possible applications we show that the problem has a solution with values in $B_{R} \subset \mathbb{R}^{3}$, whenever $u_{o}(B) \subseteq B_{R}$ and furthermore there holds

$$
\int_{\left\{\xi \in B_{R}:|H(\xi)| \geq \frac{3}{2 R}\right\}}|H|^{3} d \xi<\frac{9 \pi}{2}, \quad|H(a)| \leq \frac{1}{R} \quad \text { for } a \in \partial B_{R} .
$$

\section{INTRODUCTION}

1.1. The problem and statement of the results. The aim of this paper is to construct solutions to the Cauchy-Dirichlet problem associated to the heat flow for surfaces of prescribed mean curvature on the unit ball $B \subset \mathbb{R}^{2}$ :

$$
\left\{\begin{array}{c}
\partial_{t} u-\Delta u=-2(H \circ u) D_{1} u \times D_{2} u \quad \text { in } B \times(0, \infty), \\
u=u_{o} \quad \text { on } \partial_{\text {par }}(B \times(0, \infty)),
\end{array}\right.
$$

with solutions $u: B \times(0, \infty) \rightarrow \mathbb{R}^{3}$ and given Cauchy-Dirichlet datum $u_{o}$ and a bounded continuous function $H: \mathbb{R}^{3} \rightarrow \mathbb{R}$. As usual, by the parabolic boundary we mean $\partial_{\text {par }}(B \times(0, \infty)):=\partial B \times(0, \infty) \cup \bar{B} \times\{0\}$. The Cauchy-Dirichlet problem (1.1) features some similarities to the heat flow for harmonic maps 32, since the nonlinearity in (1.1) 1 is of critical growth and exhibits the same analytic properties as the non-linearity in the harmonic map heat flow in two spatial dimensions. Therefore, it is natural to seek necessary conditions ensuring the existence of a global weak solution of (1.1). Such conditions could be those guaranteeing existence results for the associated stationary case, i.e. for the classical Dirichlet problem related to (1.1) which was intensively studied in the early seventies. Various optimal results have been obtained, and these theorems settle the existence problem in $\mathbb{R}^{3}$. Prominent examples are the results of Heinz [17, Hildebrandt [19, 20], Gulliver and Spruck [14, 15], Steffen [30, 31] and Wente [35. Hildebrandt [19] showed that the problem can be solved in a ball $B_{R} \subset \mathbb{R}^{3}$ whenever the Dirichlet boundary

\footnotetext{
Received by the editors June 3, 2011.

2010 Mathematics Subject Classification. Primary 53A10, 58J35; Secondary 35K51, 35B40.

Key words and phrases. Surfaces of prescribed mean curvature, gradient flow, global solutions, asymptotic behavior.
} 
values $u_{o}$ and the prescribed function $H$ satisfy $\left\|u_{o}\right\|_{L^{\infty}} \leq R$ and $\|H\|_{L^{\infty}} \leq R^{-1}$. Wente 35] obtained a result in a completely different direction. If $d_{u_{o}}$ denotes the Dirichlet energy of the harmonic extension of $u_{o}$ (here we assume that such an extension is possible), then a solution of the above Dirichlet problem exists, provided $\|H\|_{L^{\infty}} \leq d \sqrt{\pi / d_{u_{o}}}$. In its best known form, that is, with a constant $d=\sqrt{2 / 3}$, the result was later achieved by Steffen [30, 31. However, Wente opened the path in this direction by giving a proof for any constant $d<\frac{1}{5}$; cf. [35, Thm. 6.2]. Finally, Steffen [30, 31] showed that the condition $\int_{\mathbb{R}^{3}}|H|^{3} d x<\frac{9 \pi}{2}$ already ensures the existence of a solution. The last two results guarantee the existence of a solution in situations where the Hildebrandt result fails. For example, when the Dirichlet datum $u_{o}$ is contained in a thin set with large diameter, the Hildebrandt result only works for $\|H\|_{L^{\infty}} \ll 1$, while the last two results allow large values of $H$. In [7] the second author and Steffen were able to improve the $H^{3}$-condition, and we will come back to this later in Theorem 9.1 when describing applications of our general existence theorem for the $H$-flow system (1.1).

When dealing with the associated parabolic problem the situation is quite different. Here it is only known that the Hildebrandt-type condition ensures the existence of a classical solution, and this goes back to Rey [26]. This result has recently been extended to higher-dimensional $H$-flows by Leone, Misawa and Verde [23], that is, to

$$
\left\{\begin{aligned}
\partial_{t} u-\operatorname{div}\left(|D u|^{n-2} D u\right) & =-\sqrt{n}^{n}(H \circ u) D_{1} u \times \ldots \times D_{n} u \quad \text { in } B \times(0, \infty), \\
u & =u_{o} \quad \text { on } \partial_{\mathrm{par}}(B \times(0, \infty))
\end{aligned}\right.
$$

for solutions $u: B \times(0, \infty) \rightarrow \mathbb{R}^{n+1}$, where now $B \subseteq \mathbb{R}^{n}$ is the unit ball in $\mathbb{R}^{n}, n \geq 2$. In the latter paper the existence of at least one global weak solution is established. In any case the methods used by the authors only allow the treatment of prescribed functions $H$ admitting a certain regularity. More precisely, the considerations are restricted to bounded Lipschitz continuous functions $H$ and thus do not provide the Hildebrandt-type result in its full generality. Nevertheless, these results are to our knowledge the only ones dealing with parabolic $H$-flows and establishing the existence of a global solution.

Without any smallness condition on the prescribed function $H$, in general one cannot expect the existence of a global solution on $B \times(0, \infty)$ due to energy concentration phenomena. When the energy concentrates it could be the case that in the weak limit a portion of the energy splits off when reaching the critical time $t \uparrow T_{*}$. At such a concentration point the solution $u(\cdot, t)$ forms a bubble which can often be recovered by a rescaling process. In [2] Chen and Levine used such a device, inspired by classical arguments from Struwe [32] introduced in the framework of the heat flow for harmonic mappings into Riemannian manifolds in the twodimensional case. We also mention a related result of Struwe 33] on the $H$-flow system in 2-dimensions with a free boundary.

The aim of the present paper is twofold: First, we are looking for a far reaching existence result for a global weak solution, which covers as an application the existence results of [26] and [23] in the case $n=2$. Here we follow an idea of Steffen, who showed that in the stationary case a certain isoperimetric condition on $H$ suffices to ensure the existence of solutions. In particular, we do not assume that the image of the Cauchy-Dirichlet datum is contained in a ball. Second, we 
remove the rather strong regularity assumption that the prescribed function is Lipschitz continuous, and attempt to set up an existence theory for the natural class of functions $H$, i.e. the class of bounded and continuous functions.

Since (1.1) 1 is the $L^{2}$-gradient flow associated with the energy functional

$$
E_{H}(u)=\frac{1}{2} \int_{B}|D u|^{2} d x+2 \mathbf{V}_{H}\left(u, u_{o}\right)
$$

where $\mathbf{V}_{H}\left(u, u_{o}\right)$ denotes the oriented $H$-volume enclosed by $u$ and the reference surface $u_{o}$, it is natural to expect existence results for the Cauchy-Dirichlet problem (1.1) under conditions similar to those from the stationary case. For the precise definition of the $H$-volume functional we refer to Section 3 . Here we only give the intuitive idea behind its definition. For the fixed reference surface $u_{o}$ and an arbitrary surface $u$ with the same boundary values as $u_{o}$, we define

$$
\mathbf{V}_{H}\left(u, u_{o}\right):=\int_{B} i_{u, u_{o}} H d \xi,
$$

where $i_{u, u_{o}}$ denotes the characteristic function of the set enclosed by the surfaces $u$ and $u_{o}$, taken with multiplicities. The $H$-volume can be interpreted as the oriented, $H$-weighted volume enclosed by two surfaces with the same boundary. In the stationary case one minimizes the energy functional $E_{H}$ corresponding to $H$ and $u_{o}$ in a closed subset $A \subseteq \mathbb{R}^{3}$. The main assumption which ensures the existence of minimizers is a certain isoperimetric condition for $H$ and $A$, which roughly says that twice the $H$-volume, that is, $2\left|\int_{E} H d \xi\right|$, of any set of finite perimeter $E \subseteq A$ is less than $c$ times the perimeter $\mathbf{P}(E)$ with a constant $0<c<1$, that is,

$$
2\left|\int_{E} H d \xi\right| \leq c \mathbf{P}(E), \quad \text { for any } E \subseteq A \text { of finite perimeter. }
$$

Under such a condition, the functional $E_{H}$ turns out to be lower semi-continuous with respect to weak convergence, and therefore one can establish a fairly general existence result which in the very end yields the above mentioned results as applications. This program has been carried out in [30, 31 for the Plateau problem for surfaces with prescribed mean curvature in $\mathbb{R}^{3}$, in [7] for the Plateau problem for surfaces with prescribed mean curvature in a Riemannian 3-manifold, and in [4] for higher-dimensional $H$-systems. We also mention the related work [6] on the Plateau problem for hypersurfaces of prescribed mean curvature in Riemannian manifolds in the framework of geometric measure theory.

As already mentioned, the present paper can be viewed as an attempt to establish a general existence result for $H$-flow systems assuming only an isoperimetric condition for $H$ and $A$. In order to explain the results we are going to prove, we begin by specifying the hypotheses. For the obstacle $A \subseteq \mathbb{R}^{3}$ we suppose that

$A \subseteq \mathbb{R}^{3}$ is closed, convex, with $C^{2}$-boundary and bounded principal curvatures.

By $\mathcal{H}_{\partial A}(a)$ we denote the minimum of the principal curvatures of $\partial A$ in the point $a \in \partial A$, taken with respect to the inward pointing unit normal vector. Moreover, we assume that

$$
H: A \rightarrow \mathbb{R} \text { is a continuous and bounded function }
$$

and satisfies

$$
|H| \leq \mathcal{H}_{\partial A} \quad \text { on } \partial A
$$


Furthermore, we assume that $H$ satisfies a spherical isoperimetric condition of type $(\boldsymbol{c}, \boldsymbol{s})$ on $\boldsymbol{A}$, for parameters $0<s \leq \infty$ and $0<c<1$. This means that for every spherical 2-current $T$ (cf. Definition 3.2) with spt $T \subseteq A$ and $\mathbf{M}(T) \leq s$, there holds

$$
2|\langle Q, H \Omega\rangle|=2\left|\int_{A} i_{Q} H \Omega\right| \leq c \mathbf{M}(T)
$$

where $Q$ denotes the unique integer multiplicity rectifiable 3-current with $\partial Q=T$, $\mathbf{M}(Q)<\infty$ and $\operatorname{spt} Q \subseteq A$. Here, $i_{Q}$ denotes the integer valued multiplicity function of $Q$ and $\Omega$ the volume form on $\mathbb{R}^{3}$. Finally, for the initial and boundary values $u_{o} \in W^{1,2}(B, A)$, we assume that they satisfy, for some parameter $1<\sigma \leq$ $\infty$

$$
(1+\sigma) \mathbf{D}\left(u_{o}\right) \leq s .
$$

Note that $\sigma=\infty$ is only possible when $s=\infty$. Under this set of assumptions, we have the following general existence result.

Theorem 1.1. Assume that $A \subseteq \mathbb{R}^{3}$ and $H: A \rightarrow \mathbb{R}$ satisfy assumptions (1.2) (1.5) and let $u_{o} \in W^{1,2}(B, A)$ be given with (1.6). Further assume that

$$
c \leq \frac{\sigma-1}{\sigma+1} \text { if } \sigma<\infty \text {. }
$$

Then there exists a weak solution

$$
u \in C^{0}\left([0, \infty) ; L^{2}(B, A)\right) \cap L^{\infty}\left((0, \infty) ; W^{1,2}(B, A)\right)
$$

with $\partial_{t} u \in L^{2}\left(B \times(0, \infty), \mathbb{R}^{3}\right)$ to the Cauchy-Dirichlet problem (1.1). The boundary values are taken in the usual trace sense, that is, $u(\cdot, t)-u_{o} \in W_{0}^{1,2}\left(B, \mathbb{R}^{3}\right)$ for almost every $t \in(0, \infty)$. Moreover, the initial datum is achieved as usual in the $L^{2}$-sense, that is, $\lim _{t \downarrow 0}\left\|u(\cdot, t)-u_{o}\right\|_{L^{2}\left(B, \mathbb{R}^{3}\right)}=0$.

Of course, Theorem 1.1 also covers the case $\sigma=\infty$ in which $s=\infty$ and $c<1$. The restriction on $c$ as in (1.7) is only needed when dealing with the case $\sigma<\infty$. As an application of Theorem 1.1 in the case when $A$ is a ball $B_{R} \subset \mathbb{R}^{3}$, we show for example that there holds: The Cauchy-Dirichlet problem (1.1) is solvable for every Dirichlet datum $u_{o} \in W^{1,2}\left(B, B_{R}\right)$ provided $H$ is bounded and continuous and

$$
\int_{\left\{\xi \in B_{R}:|H(\xi)| \geq \frac{3}{2 R}\right\}}|H|^{3} d \xi<\frac{9 \pi}{2}, \quad|H(a)| \leq \frac{1}{R} \quad \text { for } a \in \partial B_{R} .
$$

This implies, in particular, the existence of a global weak solution to (1.1) for data $u_{o} \in W^{1,2}\left(B, B_{R}\right)$ and prescribed continuous functions $H: \bar{B}_{R} \rightarrow R$ satisfying

$$
\sup _{B_{R}}|H|<\frac{3}{2 R}, \quad|H(a)| \leq \frac{1}{R} \quad \text { for } a \in \partial B_{R},
$$

and improves in the 2-dimensional case the results known to date. Further applications are given in Section 9.1. We restrict ourselves to some, but in principle the method yields the extension of all known results concerning the existence for the Dirichlet problem from the stationary case to the parabolic setting. 
The second main result is concerned with the long-time behavior of the constructed weak solution from Theorem 1.1. Here we are able to show:

Theorem 1.2. Under the assumptions of Theorem 1.1 there exist a map $u_{\infty} \in$ $W^{1,2}(B, A)$ and a sequence $t_{j} \rightarrow \infty$ such that $u\left(\cdot, t_{j}\right) \rightarrow u_{\infty}$ weakly in $W^{1,2}\left(B, \mathbb{R}^{3}\right)$ and such that $u_{\infty}$ is a solution of the Dirichlet problem

$$
\left\{\begin{array}{c}
\Delta u_{\infty}=2\left(H \circ u_{\infty}\right) D_{1} u_{\infty} \times D_{2} u_{\infty} \text { weakly in } B, \\
u_{\infty}=u_{o} \text { on } \partial B .
\end{array}\right.
$$

If the boundary values $\left.u_{o}\right|_{\partial B}$ are continuous, this solution satisfies $u_{\infty} \in C^{0}\left(\bar{B}, \mathbb{R}^{3}\right) \cap$ $C_{\mathrm{loc}}^{1, \alpha}\left(B, \mathbb{R}^{3}\right)$, and if $H$ is Hölder continuous, then $u_{\infty} \in C_{\mathrm{loc}}^{2, \alpha}\left(B, \mathbb{R}^{3}\right)$ and $u_{\infty}$ is a classical solution of (1.8).

1.2. Technical aspects and plan of the paper. For the construction of the weak solution we use a time discretization procedure that relies on ideas introduced by Kikuchi [22] and which has been used by Haga, Hoshino and Kikuchi [16] to reprove results concerning the existence of weak solutions to the harmonic map heat flow which were previously obtained by Ginzburg-Landau approximations. Further, Cai and Zhou [1] used this technique for the construction of weak solutions to nonuniformly parabolic equations and Leone, Misawa and Verde 23. in the context of the heat flow of higher-dimensional $H$-systems. Finally, with a suitable modification Moser [25] was able to deal with a biharmonic map heat flow.

In our setting the time discretization works as follows: For a fixed step size $h>0$ we sub-divide $[0, \infty)$ into $[j h,(j+1) h)$ for $j \in \mathbb{N}_{0}$. We fix a closed, convex subset $A \subseteq \mathbb{R}^{3}$ and a datum $u_{o} \in W^{1,2}(B, A)$. For $j=0$ we let $u_{o, h}:=u_{o}$. Then we define recursively time discretized energy functionals according to

$$
\mathbf{F}_{j, h}(w):=\mathbf{D}(w)+\mathbf{V}_{H}\left(w, u_{o}\right)+\frac{1}{2 h} \int_{B}\left|w-u_{j-1, h}\right|^{2} d x
$$

for $j \in \mathbb{N}$. By $u_{j, h}$ we denote a minimizer of the functional $\mathbf{F}_{j, h}$ in a fixed sub-class of $\mathcal{S}\left(u_{o}, A\right):=\left\{w \in W^{1,2}(B, A): w-u_{o} \in W_{0}^{1,2}\left(B, \mathbb{R}^{3}\right)\right\}$. This sub-class may be defined for example by a further energy restriction. Now, if it happens that the prescribed function $H: A \rightarrow \mathbb{R}$ satisfies a spherical isoperimetric condition on $A$, then by modifying the methods developed in [30 (see also 7]) the existence of a minimizer can easily be established. These minimizers are actually Hölder continuous in the interior of $B$, and if the boundary values $u_{o}$ are continuous on $\partial B$, they are moreover continuous up to the boundary $\partial B$. This can be achieved by using the $\mathbf{F}_{j, h}$-minimality following an old device of Morrey which is based on the harmonic replacement and comparison of energies. The term involving the discrete time derivative is a lower order term and therefore harmless. Nevertheless, this term causes the Hölder exponent to blow up in the sense that $\alpha \downarrow 0$ when $h \downarrow 0$, since the estimates cannot be achieved uniformly in $h$.

Due to the restriction $u_{j, h}(B) \subseteq A$ and a possible energy restriction of the form $\mathbf{D}\left(u_{j, h}\right) \leq s$, one is only able to derive an Euler-Lagrange inequality, that is, $u_{j, h}$ solves a variational inequality. This information can be used similarly as in [3, 7, 4, 6] to derive a variational equality for minimizers. This is the point where the convexity assumption on the obstacle $A$ enters. To be more precise, it turns out that the minimizers weakly solve the discrete $H$-flow system

$$
\frac{u_{j, h}-u_{j-1, h}}{h}-\Delta u_{j, h}+2\left(H \circ u_{j, h}\right) D_{1} u_{j, h} \times D_{2} u_{j, h}=\lambda_{j, h},
$$


where the right-hand side is a non-negative Radon measure supported on the coincidence set $u^{-1} \partial A$, i.e. the set of those points in $B$ where $u$ touches the obstacle $\partial A$. This Radon measure can be estimated by

$$
0 \leq \lambda_{j, h} \leq \mathcal{L}^{2}\left\llcorner\left(|H \circ u|-\mathcal{H}_{\partial A} \circ u\right)_{+}|D u|^{2} \quad \text { on } u^{-1} \partial A,\right.
$$

which means in particular that $\lambda_{j, h}$ is absolutely continuous with respect to the Lebesgue measure on $\mathbb{R}^{2}$. From the preceding estimate one easily infers that condition (1.4) implies $\lambda_{j, h} \equiv 0$ so that the geometric hypotheses imposed on $A$ lead to the assertion that $u_{j, h}$ satisfies the time discretized $H$-flow system with vanishing right-hand side.

Having arrived at this stage, one uses the sequence of minimizers $\left(u_{j, h}\right)_{j \in \mathbb{N}_{0}}$ to define an approximate solution to the $H$-flow system on the whole of $[0, \infty)$. To be more precise, one defines

$$
u_{h}(x, t):=u_{j, h}(x) \text { for } x \in B, t \in[j h,(j+1) h), j \in \mathbb{N}_{0} .
$$

Then, $u_{h}$ satisfies

$$
\Delta_{t}^{h} u_{h}-\Delta u_{h}+2\left(H \circ u_{h}\right) D_{1} u_{h} \times D_{2} u_{h}=0 \quad \text { weakly on } B \times(0, \infty),
$$

where $\Delta_{t}^{h} w(x, t)$ denotes the finite difference quotient in time, that is,

$$
\Delta_{t}^{h} w(x, t):=\frac{w(x, t)-w(x, t-h)}{h} .
$$

The main effort in this paper is to show that the solutions $u_{h}$ of the time discretized $H$-flow system converge to a solution of (1.1). Due to the non-linear character of the time discrete $H$-flow system, this is a non-trivial task and needs several technically involved tools. The major obstruction stems from the fact that the non-linear $H$ term, i.e. $2(H \circ w) D_{1} w \times D_{2} w$, is not continuous with respect to weak convergence, and here we need additional arguments to pass to the limit. For this we employ ideas used by Moser for the construction of a biharmonic map heat flow 25].

The strategy is as follows: From the uniform $L^{\infty}-W^{1,2}$-bound for $u_{h}$ and the associated bound for the $L^{2}$-norm of the discrete time derivative $\Delta_{t}^{h} u_{h}$, which come along with the approximation scheme, one can conclude that a subsequence $u_{h_{i}}$ converges in $C^{0}-L^{2}$ and weakly* in $L^{\infty}-W^{1,2}$ to a limit map $u \in L^{\infty_{-}}-W^{1,2} \cap C^{0, \frac{1}{2}}-$ $L^{2}$, and furthermore that the weak limit admits a time derivative $\partial_{t} u \in L^{2}$ and that $\Delta_{t}^{h_{i}} u_{h_{i}}$ converges weakly to $\partial_{t} u$ in $L^{2}$. But these convergence properties are not sufficient to pass to the limit in the non-linear $H$-term. Here one needs, as already mentioned, additional arguments whose core can roughly be summarized as follows: First of all one argues slice-wise, that is, for a fixed time $t$. Then the sequence $u_{h_{i}}(\cdot, t)$ is composed by different minimizers, respectively weak solutions to (1.9). By standard elliptic regularity theory one can show that these maps have locally Hölder continuous first derivatives in the interior of $B$. However, the corresponding estimate is not uniform in $h$, so the passage to the limit is not possible. Nevertheless, since now the non-linearity is locally bounded, one obtains a local $W^{2,2}$-estimate, again not uniform in $h$. This preliminary regularity can then be used to control the $L^{4}$-norm of $D u$ (which means the $L^{2}$-norm of the non-linearity) by interpolating between $W^{2,2}$ and $W^{1,2}$ by a simple application of the Gagliardo-Nirenberg interpolation lemma. This is possible on balls $B_{\varrho}\left(x_{o}\right)$ on which the Dirichlet energy of $u$ is small. For the sequence $u_{h_{i}}(\cdot, t)$ on the time slices, this means that away from isolated concentration points of the energy one obtains uniform $W^{2,2}$-estimates, since the Dirichlet energy on these balls by assumption 
does not concentrate and therefore can be made arbitrarily small, uniformly in $h$. Therefore, outside of the set of concentration points - which actually is a finite set in our setting - one can conclude strong convergence in $W^{1,2}$ on compact subsets. Together with a capacity argument this allows the passage to the limit and yields the convergence of the non-linearity along the sequence. This suffices to pass to the limit slice-wise, but the weak limit $f(\cdot, t)$ of $\Delta_{t}^{h_{i}} u_{h_{i}}(\cdot, t)$ on a fixed time slice can at this stage not be identified with the time derivative $\partial_{t} u$, which is the weak limit of $\Delta_{t}^{h_{i}} u_{h_{i}}$ in $L^{2}(B \times(0, \infty))$. We note that $f(\cdot, t)$ is constructed from an application of Lemma 7.5, and this is only possible since

$$
\sup _{i \in \mathbb{N}} \int_{B}\left|D u_{h_{i}}(\cdot, t)\right|^{2}+\left|\Delta_{t}^{h_{i}} u_{h_{i}}(\cdot, t)\right|^{2} d x<\infty
$$

for a.e. $t$. For the identification one now modifies the sequence according to the following selection principle: When the integral in the preceding estimate is bounded by a given parameter $a$, then one defines $\tilde{u}_{h_{i}}(\cdot, t) \equiv u_{h_{i}}(\cdot, t)$ and $\tilde{f}_{i}(\cdot, t) \equiv \Delta_{t}^{h_{i}} u_{h_{i}}(\cdot, t)$, while in the case that the energy bound is too large, that is, greater than $a$, one sets $\tilde{u}_{h_{i}}(\cdot, t) \equiv u(\cdot, t)$ and $\tilde{f}_{i}(\cdot, t) \equiv f(\cdot, t)$. This has the advantage that for the new sequence the convergence of the non-linear terms can be established by dominated convergence, which allows the identification of $f$ as $\partial_{t} u$.

The strategy for the proof of the asymptotic behavior is similar. First one chooses "good" time slices $t_{j} \rightarrow \infty$ and a subsequence $\mathfrak{K} \subseteq \mathbb{N}$ for which on the one hand $\int_{B}\left|\partial_{t} u\left(\cdot, t_{j}\right)\right|^{2} d x \leq \frac{1}{j}$ for all $j \in \mathbb{N}$ and on the other hand

$$
\int_{B}\left|D u_{h_{\ell}}\left(\cdot, t_{j}\right)\right|^{2}+\left|\Delta_{t}^{h_{\ell}} u_{h_{\ell}}\left(\cdot, t_{j}\right)\right|^{2} d x \leq C \quad \forall j \in \mathbb{N}, \ell \in \mathfrak{K}
$$

for a constant independent of $j$ and $\ell$. Again by an application of Lemma 7.5 one infers that for a fixed slice $t_{j}$ one has weak convergence of $\Delta_{t}^{h_{\ell}} u_{h_{\ell}}\left(\cdot, t_{j}\right)$ to $\partial_{t} u\left(\cdot, t_{j}\right)$. At this stage, for each fixed $j$ one can choose $\ell_{j}$ such that $D u_{h_{\ell_{j}}}\left(\cdot, t_{j}\right) \rightarrow D u_{\infty}$ weakly in $L^{2}\left(B, \mathbb{R}^{3 \cdot 2}\right), u_{h_{\ell_{j}}}\left(\cdot, t_{j}\right) \rightarrow u_{\infty}$ strongly in $L^{2}\left(B, \mathbb{R}^{3}\right)$ and $\Delta_{t}^{h_{\ell_{j}}} u_{h_{\ell_{j}}}\left(\cdot, t_{j}\right) \rightarrow$ 0 weakly in $L^{2}\left(B, \mathbb{R}^{3}\right)$, and this again allows - treating the non-linearity via Lemma 7.5 - the passage to the limit and proves that $u_{\infty}$ solves the stationary $H$-surface equation. Recent groundbreaking results by Rivière [27] imply moreover that the limit is $C^{1, \alpha}$-regular.

\section{Notation AND PRELIMINARIES}

2.1. Notation. By $B_{r}\left(x_{o}\right) \subset \mathbb{R}^{n}$ we denote the open ball with center $x_{o} \in \mathbb{R}^{n}$ and radius $r>0$. In the special cases $x_{o}=0$ or $r=1$ we also use the more compact notation $B_{r}=B_{r}(0)$ and $B:=B_{1}(0)$. For the mean value of a function $f \in L^{1}\left(B_{r}\left(x_{o}\right), \mathbb{R}^{k}\right)$ over $B_{r}\left(x_{0}\right)$, we abbreviate it as $f_{x_{o}, r}:=f_{B_{r}\left(x_{o}\right)} f d x$. For the Dirichlet energy of a map $u \in W^{1,2}\left(B, \mathbb{R}^{3}\right)$ we abbreviate it as

$$
\mathbf{D}(u):=\frac{1}{2} \int_{B}|D u|^{2} d x .
$$

Similarly, we write $\mathbf{D}_{G}(u):=\frac{1}{2} \int_{G}|D u|^{2} d x$ for the Dirichlet energy of $u$ restricted to a measurable subset $G \subset B$. 
2.2. Preliminaries. Next, we state two well-known elementary iteration lemmas that will be useful in what follows. The first one can e.g. be found in [12, Lemma III.2.1].

Lemma 2.1. Let $\phi(t)$ be a non-negative non-decreasing function satisfying

$$
\phi(\varrho) \leq\left[A\left(\frac{\varrho}{r}\right)^{\alpha}+\varepsilon\right] \phi(r)+B r^{\beta}
$$

whenever $0<\varrho \leq r \leq R$ with $A, B, \alpha, \beta$ non-negative constants, and $\beta<\alpha$. Then there exists $\varepsilon_{o}=\varepsilon_{o}(A, \alpha, \beta)>0$ such that if $0 \leq \varepsilon \leq \varepsilon_{o}$ we have

$$
\phi(\varrho) \leq C\left[\left(\frac{\varrho}{r}\right)^{\beta} \phi(r)+B \varrho^{\beta}\right]
$$

whenever $0<\varrho \leq r \leq R$ for a constant $C=C(A, \alpha, \beta)$.

A proof of the following lemma can be retrieved e.g. from [13, Lemma 6.1].

Lemma 2.2. For $0<r<R<\infty$, let $f:[r, R] \rightarrow[0, \infty)$ be a bounded function and assume that for all $r \leq s<t \leq R$, there holds

$$
f(s) \leq \vartheta f(t)+\frac{A}{(t-s)^{\beta}}+\frac{B}{(t-s)^{\gamma}}+C,
$$

for constants $A, B, C \geq 0,0<\gamma<\beta$ and $\vartheta \in(0,1)$. Then we have

$$
f(r) \leq c(\beta, \vartheta)\left[\frac{A}{(R-r)^{\beta}}+\frac{B}{(R-r)^{\gamma}}+C\right] .
$$

A key tool for the proof of the regularity and thereby the strong convergence of approximating solutions is the following Gagliardo-Nirenberg-type interpolation inequality.

Lemma 2.3. Let $B_{\varrho}\left(x_{o}\right) \subset \mathbb{R}^{n}$ with $0<\varrho \leq 1,1 \leq \sigma, q, r<\infty$ and $\vartheta \in(0,1)$ such that $-\frac{n}{\sigma} \leq \vartheta\left(1-\frac{n}{q}\right)-(1-\vartheta) \frac{n}{r}$. Then there exists a constant $c=c(n, q, r)$ such that for any $u \in W^{1, q}\left(B_{\varrho}\left(x_{o}\right)\right)$ there holds

$$
f_{B_{\varrho}\left(x_{o}\right)}\left|\frac{u}{\varrho}\right|^{\sigma} d x \leq c\left(f_{B_{\varrho}\left(x_{o}\right)}\left|\frac{u}{\varrho}\right|^{q}+|D u|^{q} d x\right)^{\frac{\vartheta \sigma}{q}}\left(f_{B_{\varrho}\left(x_{o}\right)}\left|\frac{u}{\varrho}\right|^{r} d x\right)^{\frac{(1-\vartheta) \sigma}{r}} .
$$

We apply this to $|D u| \in W^{1,2}\left(B_{\varrho}\left(x_{o}\right)\right)$ for a map $u \in W^{2,2}\left(B_{\varrho}\left(x_{o}\right), \mathbb{R}^{N}\right)$ for the choice $\sigma=4, n=2, q=r=2, \vartheta=\frac{1}{2}$ and obtain, with a universal constant $C$, the following interpolation estimate:

$$
\int_{B_{\varrho}\left(x_{o}\right)}|D u|^{4} d x \leq C \int_{B_{\varrho}\left(x_{o}\right)}\left|D^{2} u\right|^{2}+\left|\frac{D u}{\varrho}\right|^{2} d x \int_{B_{\varrho}\left(x_{o}\right)}|D u|^{2} d x .
$$

The following selection result is due to Eisen 8 .

Lemma 2.4. Let $G \subseteq \mathbb{R}^{n}$ be a measurable set with $\mathcal{L}^{n}(G)<\infty$. Assume that $\left\{M_{j}\right\}_{j \in \mathbb{N}}$ is a sequence of measurable sets $M_{j} \subseteq G$ such that, for some $\varepsilon>0$, the following estimate holds:

$$
\mathcal{L}^{n}\left(M_{j}\right)>\varepsilon \quad \forall j \in \mathbb{N} .
$$

Then there exists a subsequence $\left(M_{j_{\ell}}\right)_{\ell \in \mathbb{N}}$ such that

$$
\bigcap_{\ell \in \mathbb{N}} M_{j_{\ell}} \neq \varnothing \text {. }
$$




\section{The $H$-volume functional}

In this section we recall the definitions and properties of the $H$-volume functional. The material is taken from [30, respectively [7. We begin by recalling some basic terminology concerning currents. The standard references for their theory are [10] and 29 .

By $\mathcal{D}^{k}\left(\mathbb{R}^{3}\right), k \in\{0,1,2,3\}$, we denote the space of smooth $k$-forms with compact support in $\mathbb{R}^{3}$. A $k$-current on $\mathbb{R}^{3}$ is a distribution $T: \mathcal{D}^{k}\left(\mathbb{R}^{3}\right) \rightarrow \mathbb{R}$. The mass of $T$ is defined by

$$
\mathbf{M}(T):=\sup \left\{T(\omega): \omega \in \mathcal{D}^{k}\left(\mathbb{R}^{3}\right),\|\omega\|_{\infty} \leq 1\right\} .
$$

The boundary of a general $k$-current $T$ is defined by $\partial T(\alpha):=T(d \alpha)$ for $\alpha \in$ $\mathcal{D}^{k-1}\left(\mathbb{R}^{3}\right)$. A current $T$ is called closed if $\partial T \equiv 0$.

In this paper we will deal only with a special kind of current, which we define next.

Definition 3.1. A $k$-current $T$ on $\mathbb{R}^{3}$ is called an integer multiplicity rectifiable $k$-current if it can be written as

$$
T(\omega):=\int_{M}\langle\omega(x), \xi(x)\rangle \theta(x) d \mathcal{H}^{k}(x) \quad \forall \omega \in \mathcal{D}^{k}\left(\mathbb{R}^{3}\right),
$$

where $\mathcal{H}^{k}$ denotes the $k$-dimensional Hausdorff-measure, $M \subset \mathbb{R}^{3}$ is an $\mathcal{H}^{k}$-measurable, countably $k$-rectifiable subset, $\theta: M \rightarrow \mathbb{N}$ is a locally $\mathcal{H}^{k}$-integrable function and $\xi: M \rightarrow \wedge_{k}\left(\mathbb{R}^{3}\right)$ is an $\mathcal{H}^{k}$-measurable function of the form $\xi(x)=\tau_{1}(x) \wedge \ldots \wedge$ $\tau_{k}(x)$, where for $\mathcal{H}^{k}$-a.e. $x \in M, \tau_{1}(x), \ldots, \tau_{k}(x)$ form an orthonormal basis of the approximate tangent space $T_{x} M$.

We point out that in this definition we follow the terminology of Simon [29], which differs from the one of Federer [10. In the language of Federer, the currents defined above are called locally rectifiable currents.

Given $u \in W^{1,2}\left(B, \mathbb{R}^{3}\right)$ we define the associated 2-current $J_{u}$ on $\mathbb{R}^{3}$ via integration of 2 -forms over the surface $u$; that is,

$$
J_{u}(\omega):=\int_{B} u^{\#} \omega=\int_{B}\left\langle\omega \circ u, D_{1} u \wedge D_{2} u\right\rangle d x \quad \forall \omega \in \mathcal{D}^{2}\left(\mathbb{R}^{3}\right) .
$$

The current $J_{u}$ is a 2-current of finite mass, since

$$
\mathbf{M}\left(J_{u}\right):=\sup \left\{J_{u}(\omega): \omega \in \mathcal{D}^{2}\left(\mathbb{R}^{3}\right),\|\omega\|_{\infty} \leq 1\right\} \leq \int_{B}\left|D_{1} u \wedge D_{2} u\right| d x \leq \mathbf{D}(u) .
$$

A Lusin-type approximation argument as in 9, Sect. 6.6.3] shows that $J_{u}$ is an integer multiplicity rectifiable 2 -current in $\mathbb{R}^{3}$. If $v \in W^{1,2}\left(B, \mathbb{R}^{3}\right)$ is a parametric surface with associated 2-current $J_{v}$, then $\left(J_{u}-J_{v}\right)(\omega)$ is determined by integration of $u^{\#} \omega-v^{\#} \omega$ over the set $G:=\{x \in B: u(x) \neq v(x)\}$, and therefore we have

$$
\mathbf{M}\left(J_{u}-J_{v}\right) \leq \mathbf{D}_{G}(u)+\mathbf{D}_{G}(v) .
$$

For surfaces $u, v \in W^{1,2}\left(B, \mathbb{R}^{3}\right)$ with $u-v \in W_{0}^{1,2}\left(B, \mathbb{R}^{3}\right)$ we have $\partial\left(J_{u}-J_{v}\right)=0$, i.e. $\quad J_{u}-J_{v}$ is a closed 2-current in $\mathbb{R}^{3}$. This can easily be seen for surfaces $u, v \in C^{2}\left(\bar{B}, \mathbb{R}^{3}\right)$ with $u=v$ on $\partial B$. The general case follows by a standard approximation argument. 
Now we consider a closed convex obstacle $A \subseteq \mathbb{R}^{3}$ and a fixed reference surface $u_{o} \in W^{1,2}(B, A)$. Then we denote by

$$
\mathcal{S}\left(u_{o}, A\right):=\left\{u \in W^{1,2}(B, A): u-u_{o} \in W_{0}^{1,2}\left(B, \mathbb{R}^{3}\right)\right\}
$$

the set of admissible surfaces. The main idea for the geometric construction of the $H$-volume $\mathbf{V}_{H}(u, v)$ enclosed by two surfaces $u, v \in W^{1,2}(B, A)$ is to consider an integer multiplicity rectifiable 3 -current $Q$ of finite mass in $\mathbb{R}^{3}$ with boundary $\partial Q=J_{u}-J_{v}$ and to integrate $H \Omega$ over $Q$, where $\Omega$ stands for the standard volume form on $\mathbb{R}^{3}$. Such 3 -currents have a simple structure, namely they are representable by a function $i_{Q} \in L^{1}\left(\mathbb{R}^{3}, \mathbb{Z}\right)$ such that there holds

$$
Q(\gamma)=\int_{\mathbb{R}^{3}} i_{Q} \gamma \quad \forall \gamma \in \mathcal{D}^{3}\left(\mathbb{R}^{3}\right)
$$

The integer valued function $i_{Q}$ can be interpreted as a set with integer multiplicities and finite (absolute) volume. In the setting from above, the condition $\partial Q=J_{u}-J_{v}$ means that the mappings $u$ and $v$ parametrize the boundary of this set where the multiplicities are taken into account. Of course, this has to be understood in duality to the Stokes theorem, that is,

$$
\int_{\mathbb{R}^{3}} i_{Q} d \omega=\int_{B} u^{\#} \omega-\int_{B} v^{\#} \omega \quad \forall \omega \in \mathcal{D}^{2}\left(\mathbb{R}^{3}\right) .
$$

Since $\partial Q$ has finite mass the multiplicity function $i_{Q}$ is actually a BV-function on $\mathbb{R}^{3}$. The considerations from above altogether suggest that we define the $H$-volume by

$$
\mathbf{V}_{H}(u, v):=\int_{\mathbb{R}^{3}} i_{Q} H \Omega .
$$

To make this well defined we need the uniqueness of $Q$ in the previous construction. But this is a consequence of the constancy theorem, which ensures that any 3current with boundary $J_{u}-J_{v}$ is unique up to integer multiples of $\llbracket \mathbb{R}^{3} \rrbracket$, and hence the 3-current $Q$ with finite mass and boundary $J_{u}-J_{v}$ is unique. To proceed further we need the following.

Definition 3.2. A 2-current $T$ with support in $A$ is called spherical iff it can be represented by a map $f \in W^{1,2}\left(S^{2}, A\right)$ in the form $T=f_{\#} \llbracket S^{2} \rrbracket$, i.e.

$$
T(\omega)=\int_{S^{2}} f^{\#} \omega \quad \forall \omega \in \mathcal{D}^{2}\left(\mathbb{R}^{3}\right) .
$$

From [7, Lemma 3.3] we recall the following fact concerning 2-currents of the form $J_{u}-J_{v}$ (cf. also [4]):

Lemma 3.3. For any $u, v \in W^{1,2}(B, A)$ with $u-v \in W_{0}^{1,2}\left(B, \mathbb{R}^{3}\right)$, the current $J_{u}-J_{v}$ is a spherical 2-current in $A$.

If $T$ is a spherical 2-current in $A$ (which implies in particular that $T$ is a closed, integer multiplicity rectifiable 2 current of finite mass and $\operatorname{spt} T \subseteq A$ ), then there exists an integer multiplicity rectifiable 3 -current $Q$ of finite mass with $\partial Q=T$ by the deformation theorem (see [29, Thm. 29.1] or [10, 4.2.9]). As before, we conclude by the constancy theorem that $Q$ is unique. In order to prove spt $Q \subseteq A$, we consider the nearest-point-retraction $\pi: \mathbb{R}^{3} \rightarrow A$, which exists since $A$ is convex. 
From the equality $\partial \pi_{\#} Q=\pi_{\#} \partial Q=T$ and $\mathbf{M}\left(\pi_{\#} Q\right) \leq(\operatorname{Lip} \pi)^{3} \mathbf{M}(Q) \leq \mathbf{M}(Q)$, we infer $\pi_{\#} Q=Q$, that is, spt $Q \subseteq A$. Summarizing, we have shown

Lemma 3.4. Let $A \subseteq \mathbb{R}^{3}$ be a closed convex set. Then for every spherical 2 -current $T$ on $\mathbb{R}^{3}$ with spt $T \subseteq A$, there exists a unique integer multiplicity rectifiable 3 current $Q$ with $\mathbf{M}(Q)<\infty, \partial Q=T$ and $\operatorname{spt} Q \subseteq A$.

We use this fact to define the oriented $H$-volume enclosed by two maps $u, v \in$ $W^{1,2}(B, A)$ with the same boundary values.

Definition 3.5. Let $u, v \in W^{1,2}(B, A)$ with $u-v \in W_{0}^{1,2}\left(B, \mathbb{R}^{3}\right), J_{u}-J_{v}$ be the associated spherical 2-current and $I_{u, v}$ be the unique integer multiplicity rectifiable 3-current with boundary $\partial I_{u, v}=J_{u}-J_{v}$, finite mass $\mathbf{M}\left(I_{u, v}\right)<\infty$ and spt $I_{u, v} \subseteq A$. Then the $H$-volume enclosed by $u$ and $v$ is defined by

$$
\mathbf{V}_{H}(u, v):=I_{u, v}(H \Omega)=\int_{\mathbb{R}^{3}} i_{u, v} H \Omega .
$$

Here $i_{u, v}$ stands for the multiplicity function of $I_{u, v}$.

We note that if $H$ satisfies a spherical isoperimetric condition of type $(c, s)$ on $A$ as defined in (1.5), then for any $u, v \in W^{1,2}(B, A)$ with $u-v \in W_{0}^{1,2}\left(B, \mathbb{R}^{3}\right)$ and $\mathbf{M}\left(J_{u}-J_{v}\right) \leq s$, we can bound the $H$-volume $\mathbf{V}_{H}(u, v)$ by

$$
2\left|\mathbf{V}_{H}(u, v)\right| \leq c \mathbf{M}\left(J_{u}-J_{v}\right) \leq c\left(\mathbf{D}_{G}(u)+\mathbf{D}_{G}(v)\right),
$$

where $G=\{x \in B: u(x) \neq v(x)\}$. The second inequality follows easily from (3.1).

Finally, we state some properties of the $H$-functional that were used in the literature to construct solutions of the stationary $H$-system; see [7, Lemma 3.6] or [4, Lemma 3.5]. Here we will apply them to a time discretized problem in order to construct solutions of the heat flow for the $H$-system.

Lemma 3.6. Let $u, v \in W^{1,2}(B, A)$ with $u-v \in W_{0}^{1,2}\left(B, \mathbb{R}^{3}\right)$ so that the $H$-volume $\mathbf{V}_{H}(u, v)$ is defined (cf. Definition 3.5).

(i) Assume that $\tilde{u} \in W^{1,2}(B, A)$ is given with $u-\tilde{u} \in W_{0}^{1,2}\left(B, \mathbb{R}^{3}\right)$. Then $\mathbf{V}_{H}(\tilde{u}, v)$ and $\mathbf{V}_{H}(\tilde{u}, u)$ (that are also well defined by Lemma 3.4) satisfy

$$
\mathbf{V}_{H}(\tilde{u}, u)+\mathbf{V}_{H}(u, v)=\mathbf{V}_{H}(\tilde{u}, v)
$$

and

$$
\left|\mathbf{V}_{H}(\tilde{u}, u)\right| \leq\|H\|_{L^{\infty}}\|u-\tilde{u}\|_{L^{\infty}}\left[\mathbf{D}_{G}(u)+\mathbf{D}_{G}(\tilde{u})\right],
$$

where $G=\{x \in B: u(x) \neq \tilde{u}(x)\}$.

(ii) Let $\Phi_{\tau}^{Y}$ be the flow of a vector-field $Y \in C_{0}^{1}\left(\mathbb{R}^{3}, \mathbb{R}^{3}\right)$ with $\Phi_{\tau}^{Y}(A) \subseteq A$ for small $\tau>0,0 \leq \eta \in C_{0}^{1}(B, \mathbb{R})$ and $u_{\tau}(x):=U(\tau, x)=\Phi^{Y}(\tau \eta(x), u(x))$. Then $\mathbf{V}_{H}\left(u_{\tau}, u\right)$ and $\mathbf{V}_{H}\left(u_{\tau}, v\right)$ are defined for small $\tau>0$ and the following homotopy formula holds:

$$
\begin{aligned}
\mathbf{V}_{H}\left(u_{\tau}, v\right)-\mathbf{V}_{H}(u, v) & =\mathbf{V}_{H}\left(u_{\tau}, u\right) \\
& =\int_{B} \int_{0}^{\tau}(H \circ U)\left\langle\Omega \circ U, U_{s} \wedge U_{x_{1}} \wedge U_{x_{2}}\right\rangle d s d x .
\end{aligned}
$$




\section{The time Discrete VARIATIONAL FORMulation}

To set up the approximation scheme by time discretization we will use $H$-energy functionals of the form

$$
\mathbf{F}(u):=\mathbf{D}(u)+2 \mathbf{V}_{H}\left(u, u_{o}\right)+\frac{1}{2 h} \int_{B}|u-z|^{2} d x
$$

defined for $u \in W_{0}^{1,2}\left(B, \mathbb{R}^{3}\right)$ with $u-u_{o} \in W^{1,2}\left(B, \mathbb{R}^{3}\right)$; see Definition 3.5 for the definition of the volume functional. Here, $h>0$ and $z \in W^{1,2}\left(B, \mathbb{R}^{3}\right)$ with $z-u_{o} \in W_{0}^{1,2}\left(B, \mathbb{R}^{3}\right)$ are given. The $H$-volume term measures in a certain sense the oriented and $H$-weighted volume enclosed by the surface $u$ and a given fixed reference surface $u_{o}$.

We begin by calculating the first variation of $\mathbf{F}$. We call $u_{\tau}$ a sufficiently regular variation if $u_{\tau} \in W^{1,2}\left(B, \mathbb{R}^{3}\right)$ for $|\tau|$ sufficiently small, $u_{0}=u$, the initial vector-field satisfies $\varphi=\left.\frac{d}{d \tau}\right|_{\tau=0} u_{\tau} \in L^{\infty}\left(B, \mathbb{R}^{3}\right) \cap W_{0}^{1,2}\left(B, \mathbb{R}^{3}\right)$, and if the formal differentiation under the integral with respect to $\tau$ at $\tau=0$ is allowed in $\mathbf{D}\left(u_{\tau}\right)$ and in $\mathbf{V}_{H}\left(u_{\tau}, u_{o}\right)-\mathbf{V}_{H}\left(u, u_{o}\right)$.

Lemma 4.1. For any sufficiently regular variation $u_{\tau}$ of $u \in W^{1,2}\left(B, \mathbb{R}^{3}\right)$ with initial vector-field $\varphi \in L^{\infty}\left(B, \mathbb{R}^{3}\right) \cap W_{0}^{1,2}\left(B, \mathbb{R}^{3}\right)$, there holds

$$
\left.\frac{d}{d \tau}\right|_{\tau=0} \mathbf{F}\left(u_{\tau}\right)=\int_{B}\left[\frac{u-z}{h}+D u \cdot D \varphi+2(H \circ u) \varphi \cdot D_{1} u \times D_{2} u\right] d x .
$$

Proof. The formal differentiation of $\frac{1}{2 h} \int_{B}\left|u_{\tau}-z\right|^{2} d x$ and of $\mathbf{D}\left(u_{\tau}\right)$ give the first two terms on the right-hand side of (4.2). For the volume term, we employ the homotopy formula as in (3.3). With the abbreviation $U(\tau, x):=u_{\tau}(x)$, this gives

$$
2 \mathbf{V}_{H}\left(u_{\tau}, u_{o}\right)-2 \mathbf{V}_{H}\left(u, u_{o}\right)=2 \int_{0}^{\tau} \int_{B}(H \circ U)\left\langle\Omega, U_{s} \wedge U_{x_{1}} \wedge U_{x_{2}}\right\rangle d x d s
$$

Differentiating leads to the integrand $2(H \circ u)\left\langle\Omega, \varphi \wedge D_{1} u \wedge D_{2} u\right\rangle=2(H \circ u) \varphi$. $D_{1} u \times D_{2} u$, which is the last term in (4.2).

The integral $\delta \mathbf{F}(u ; \varphi)$ in (4.2) is called the first variation of the functional $\mathbf{F}$ in direction $\varphi$. The preceding lemma leads to the following.

Definition 4.2. We say that a function $u \in W^{1,2}\left(B, \mathbb{R}^{3}\right)$ is a weak solution to the time discrete evolutionary $H$-surface system if and only if $\delta \mathbf{F}(u ; \varphi)=0$ for all vector-fields $\varphi \in L^{\infty}\left(B, \mathbb{R}^{3}\right) \cap W_{0}^{1,2}\left(B, \mathbb{R}^{3}\right)$, i.e. if there holds

$$
\int_{B}\left[\frac{u-z}{h} \cdot \varphi+D u \cdot D \varphi+2(H \circ u) \varphi \cdot D_{1} u \times D_{2} u\right] d x=0
$$

for all $\varphi \in L^{\infty}\left(B, \mathbb{R}^{3}\right) \cap W_{0}^{1,2}\left(B, \mathbb{R}^{3}\right)$.

Various types of admissible variations have been used in the literature to deduce the Euler-Lagrange system for the $H$-surface system. The most convenient ones for dealing with obstacle problems are variations of the form

$$
u_{\tau}(x):=\Phi^{Y}(\tau \eta(x), u(x)),
$$

where $\Phi^{Y}$ is the flow associated to a smooth vector-field $Y \in C_{0}^{1}\left(\mathbb{R}^{3}, \mathbb{R}^{3}\right)$ and $\eta \in C_{0}^{1}(B, \mathbb{R})$. 
Lemma 4.3. (i) Assume that $u \in W^{1,2}\left(B, \mathbb{R}^{3}\right)$ is $\mathbf{F}$-minimizing with respect to variations $u_{\tau}$ with $|\tau| \ll 1$ of the type described in (4.5) for each vector-field $Y \in$ $C_{0}^{1}\left(\mathbb{R}^{3}, \mathbb{R}^{3}\right)$ and each $\eta \in C_{0}^{1}(B, \mathbb{R})$. Then $u$ is a weak solution of the time discrete evolutionary $H$-surface system (4.4).

(ii) Let $A \subseteq \mathbb{R}^{3}$ be the closure of a convex $C^{2}$-domain in $\mathbb{R}^{3}$. Assume furthermore that $u$ is $\mathbf{F}$-minimizing for one-sided variations $u_{\tau}, 0 \leq \tau \ll 1$, as defined above for $\eta \geq 0$ and that $Y \in C_{0}^{1}\left(\mathbb{R}^{3}, \mathbb{R}^{3}\right)$ satisfies either $Y(a)=0$ or $Y(a)$ is directed strictly in the interior of $A$ at every $a \in \partial A$. Then $u$ is a solution to the following variational inequality:

$$
\delta \mathbf{F}(u ; \varphi)=\int_{B}\left[\frac{u-z}{h} \cdot \varphi+D u \cdot D \varphi+2(H \circ u) \varphi \cdot D_{1} u \times D_{2} u\right] d x \geq 0,
$$

for all vector-fields $\varphi \in L^{\infty}\left(B, \mathbb{R}^{3}\right) \cap W_{0}^{1,2}\left(B, \mathbb{R}^{3}\right)$ satisfying $\varphi \cdot(\tilde{\nu} \circ u) \geq 0$ almost everywhere on $u^{-1} V$ for some neighborhood $V$ of $\partial A$ in $\mathbb{R}^{3}$ and some $C^{1}$-vector-field $\tilde{\nu}$ on $\mathbb{R}^{3}$ extending the inner unit normal vector-field $\nu$ of $\partial A$.

Proof. (i) It is easy to verify that the variation $u_{\tau}$ is sufficiently regular in the sense described after (4.3) so that (4.4) holds for the variational vector-field $\varphi=\eta(Y \circ u)$ with $\eta \in C_{0}^{1}(B, \mathbb{R})$. By a standard approximation argument we can allow $\eta(Y \circ u)$ in (4.4) with $Y$ as before but with $\eta \in L^{\infty}(B, \mathbb{R}) \cap W_{0}^{1,2}(B, \mathbb{R})$. Now we consider $\psi \in C_{\mathrm{cpt}}^{\infty}\left(\mathbb{R}^{3}\right)$ and choose vector-fields $Y_{i} \in C_{0}^{1}\left(\mathbb{R}^{3}, \mathbb{R}^{3}\right)$ with $Y_{i} \equiv e_{i}$ on spt $\psi$ for $i=$ $1,2,3$. Given a general vector-field $\varphi \in L^{\infty}\left(B, \mathbb{R}^{3}\right) \cap W_{0}^{1,2}\left(B, \mathbb{R}^{3}\right)$ as allowed in (4.4), we then write $(\psi \circ u) \varphi=\sum_{i=1}^{3} \eta_{i} e_{i}$ with $\eta_{i}:=(\psi \circ u) \varphi \cdot e_{i} \in L^{\infty}(B, \mathbb{R}) \cap W_{0}^{1,2}(B, \mathbb{R})$ so that $(\psi \circ u) \varphi$ is admissible in (4.4). Finally, we approximate the constant function 1 on $\mathbb{R}^{3}$ in a suitable way with functions $\psi \in C_{\mathrm{cpt}}^{\infty}\left(\mathbb{R}^{3}\right)$ and obtain (4.4) for general $\varphi \in L^{\infty}\left(B, \mathbb{R}^{3}\right) \cap W_{0}^{1,2}\left(B, \mathbb{R}^{3}\right)$.

(ii) As in (i) we derive that $\delta \mathbf{F}(u ; \varphi) \geq 0$ holds for $\varphi=\eta(Y \circ u)$ with $\eta \geq 0, \eta \in$ $L^{\infty}(B, \mathbb{R}) \cap W_{0}^{1,2}(B, \mathbb{R})$ and $Y$ fulfilling the requirements on $\partial A$, that is, $Y(a)=0$ or $Y(a)$ is directed strictly inwards at each $a \in \partial A$. Moreover, for vector-fields $Y$ which are tangential to $\partial A$ along $\partial A$ we have $\delta \mathbf{F}(u ; \eta(Y \circ u))=0$, because in this case $-Y$ is also admissible. In this case the assumption $\eta \geq 0$ may be eliminated. For general vector-fields $\varphi$ as in (ii) we proceed as follows: For smooth functions $\psi: \mathbb{R}^{3} \rightarrow[0, \infty)$ with support sufficiently small intersecting $\partial A$, we use the previous argument with $Y_{1}=\tilde{\nu}$ and $Y_{2}, Y_{3}$ tangential along $\partial A$ (and $Y_{1}, Y_{2}, Y_{3}$ linearly independent, of course) to deduce for $\eta \geq 0$ that there holds $\delta \mathbf{F}(u ;(\psi \circ u) \varphi) \geq 0$. On the other hand, when spt $\psi \cap \partial A=\varnothing$ we have (see the argument from part (i)) that $\delta \mathbf{F}(u ;(\psi \circ u) \varphi)=0$. At this stage we can use a partition of unity argument to conclude $\delta \mathbf{F}(u ; \varphi) \geq 0$. This finishes the proof of the lemma.

Lemma 4.4. Let $A \subseteq \mathbb{R}^{3}$ be the closure of a convex $C^{2}$-domain in $\mathbb{R}^{3}$ and assume that the principal curvatures of $\partial A$ are bounded. Moreover, let $\nu$ be the inner unit normal along $\partial A$ and let $\mathcal{H}_{\partial A}(a)$ be the minimum of the principle curvatures of $\partial A$ at the point a with respect to $\nu$. Let $u \in W^{1,2}(B, A)$ satisfy the variational inequality (4.6). Then the following assertions hold:

(i) There exists a non-negative Radon measure $\lambda$ on $B$ which is absolutely continuous with respect to $\mathcal{L}^{2}$ and which is concentrated on the coincidence set $u^{-1} \partial A$ 
such that

$$
\delta \mathbf{F}(u ; \varphi)=\int_{u^{-1} \partial A} \varphi \cdot(\nu \circ u) d \lambda
$$

for each $\varphi \in L^{\infty}\left(B, \mathbb{R}^{3}\right) \cap W_{0}^{1,2}\left(B, \mathbb{R}^{3}\right)$.

(ii) For the Radon measure $\lambda$ we have the estimate

$$
\lambda \leq \mathcal{L}^{2}\left\llcorner\left(|H \circ u|-\mathcal{H}_{\partial A} \circ u\right)_{+}|D u|^{2} \quad \text { on } u^{-1} \partial A .\right.
$$

(iii) If we further impose the condition that

$$
|H(a)| \leq \mathcal{H}_{\partial A}(a)
$$

along $\partial A$, we have $\lambda \equiv 0$.

Proof. We write $d(p):=\operatorname{dist}(p, \partial A)$ for the distance of $p \in \mathbb{R}^{3}$ to $\partial A$, and we extend $\nu$ to a bounded $C^{1}$-vector-field on $\mathbb{R}^{3}$ which we still denote by $\nu$ and which coincides with $\nabla d$ on an $\varepsilon$-neighborhood of $\partial A$. This is possible for a sufficiently small $\varepsilon>0$ by our curvature assumptions on the boundary of the convex set $A$. Then $\varphi=\eta(\nu \circ u)$ is admissible in the variational inequality (4.6) provided $0 \leq$ $\eta \in C_{0}^{1}(B, \mathbb{R})$. Next, we choose $\vartheta \in C^{\infty}(\mathbb{R}, \mathbb{R})$ non-increasing with spt $\vartheta \subseteq(-\infty, 1)$ and $\operatorname{spt}(1-\vartheta) \subseteq(0, \infty)$. We define $\vartheta_{\varepsilon}(s):=\vartheta\left(\varepsilon^{-1} s\right)$ for $\varepsilon>0$ as chosen above and consider the vector-field $\varphi_{\varepsilon}:=\eta\left(\vartheta_{\varepsilon} \circ d \circ u\right)(\nu \circ u)$ with $\eta \geq 0$ as above. Then $\varphi_{\varepsilon}=\varphi$ holds on the preimage of a neighborhood of $\partial A$, and therefore $\varphi-\varphi_{\varepsilon}$ and $\varphi_{\varepsilon}-\varphi$ are both admissible in the variational inequality so that

$$
\delta \mathbf{F}\left(u ; \varphi_{\varepsilon}\right)=\delta \mathbf{F}(u ; \varphi) \geq 0 .
$$

For $0<\varepsilon \ll 1$ and $i=1,2$ we estimate

$$
\begin{aligned}
D_{i} u \cdot D_{i} \varphi_{\varepsilon}= & D_{i} \eta\left(\vartheta_{\varepsilon} \circ d \circ u\right) D_{i} u \cdot(\nu \circ u)+ \\
& \eta\left(\vartheta_{\varepsilon}^{\prime} \circ d \circ u\right)\left(D_{i} u \cdot(\nu \circ u)\right)^{2} \\
& +\eta\left(\vartheta_{\varepsilon} \circ d \circ u\right) D_{i} u \cdot((D \nu) \circ u) D_{i} u \\
\leq & \left(\vartheta_{\varepsilon} \circ d \circ u\right)\left[D_{i} \eta D_{i} u \cdot(\nu \circ u)+\eta D_{i} u \cdot((D \nu) \circ u) D_{i} u\right] .
\end{aligned}
$$

Summing with respect to $i=1,2$ we obtain

$$
\begin{aligned}
0 \leq & \delta \mathbf{F}(u ; \varphi)=\delta \mathbf{F}\left(u ; \varphi_{\varepsilon}\right) \\
\leq & \int_{B}\left[\eta \frac{u-z}{h}+D_{i} \eta D_{i} u\right] \cdot(\nu \circ u)\left(\vartheta_{\varepsilon} \circ d \circ u\right) d x \\
& +\int_{B}\left[2(H \circ u)(\nu \circ u) \cdot D_{1} u \times D_{2} u-(B \circ u)\left(D_{i} u, D_{i} u\right)\right]\left(\vartheta_{\varepsilon} \circ d \circ u\right) \eta d x \\
\leq & \int_{B}\left[\eta \frac{u-z}{h}+D_{i} \eta D_{i} u\right] \cdot(\nu \circ u)\left(\vartheta_{\varepsilon} \circ d \circ u\right) d x \\
& +\int_{B}\left[|H \circ u \| D u|^{2}-(B \circ u)\left(D_{i} u, D_{i} u\right)\right]\left(\vartheta_{\varepsilon} \circ d \circ u\right) \eta d x,
\end{aligned}
$$

where we abbreviated

$$
B(p)(\xi, \zeta):=-\xi \cdot D \nu(p) \zeta \quad \text { for all } \xi, \zeta \in \mathbb{R}^{3} .
$$


Since the left-hand side of (4.10) is independent of $\varepsilon$, we can pass to the limit $\varepsilon \downarrow 0$. Taking into account the identity $D_{i} u \cdot(\nu \circ u)=0$ on $u^{-1} \partial A$, we arrive at

$$
\begin{aligned}
0 & \leq \delta \mathbf{F}(u, \eta(\nu \circ u)) \\
& \leq \int_{u^{-1} \partial A}\left[\frac{u-z}{h} \cdot(\nu \circ u)+|H \circ u||D u|^{2}-\sum_{i=1}^{2} b_{\partial A} \circ u\left(D_{i} u, D_{i} u\right)\right] \eta d x \\
& \leq \int_{u^{-1} \partial A}\left[\frac{u-z}{h} \cdot(\nu \circ u)+\left(|H \circ u|-\mathcal{H}_{\partial A} \circ u\right)|D u|^{2}\right] \eta d x .
\end{aligned}
$$

Here we used in the second-to-last line that $(B \circ u)\left(D_{i} u, D_{i} u\right)=\left(b_{\partial A} \circ u\right)\left(D_{i} u, D_{i} u\right)$ almost everywhere on $u^{-1} \partial A$, where $b_{\partial A}$ denotes the second fundamental form of $\partial A$ in $\mathbb{R}^{3}$, and in the last line we used the definition of $\mathcal{H}_{\partial A}$. Because of the preceding estimate, the Riesz representation theorem yields a non-negative Radon measure $\lambda$ on $B$, concentrated on $u^{-1} \partial A$, such that

$$
\delta \mathbf{F}(u ; \eta(\nu \circ u))=\int_{B} \eta d \lambda
$$

for all $\eta \in C_{0}^{1}(B, \mathbb{R})$. Moreover, this Radon measure satisfies the estimate

$$
0 \leq \lambda \leq\left[\frac{u-z}{h} \cdot(\nu \circ u)+\left(|H \circ u|-\mathcal{H}_{\partial A} \circ u\right)|D u|^{2}\right]_{+} \quad \text { on } u^{-1} \partial A .
$$

Since $A$ is convex we have $(u(x)-z(x)) \cdot \nu(u(x)) \leq 0$ for a.e. $x \in u^{-1} \partial A$, since $z(x) \in A$ and $u(x) \in \partial A$, so $u(x)-z(x) \in\left\{p \in \mathbb{R}^{3}: p \cdot \nu(u(x)) \leq 0\right\}$. Hence, we conclude that

$$
0 \leq \int_{B} \eta d \lambda \leq \int_{u^{-1} \partial A}\left(|H \circ u|-\mathcal{H}_{\partial A} \circ u\right)_{+}|D u|^{2} \eta d x
$$

yielding the claim (4.8) and finishing the proof of assertion (ii).

To proof (i) we start by noting that (ii), i.e. (4.8), yields the absolute continuity of the Radon measure $\lambda$ with respect to $\mathcal{L}^{2}$ and, moreover, the estimate $\lambda(B \backslash$ $\left.u^{-1} \partial A\right)=0$. By approximation it is easy to check that (4.11) holds for $\eta \in$ $L^{\infty}(B, \mathbb{R}) \cap W_{0}^{1,2}(B, \mathbb{R})$. Now, a general vector-field $\varphi \in L^{\infty}\left(B, \mathbb{R}^{3}\right) \cap W_{0}^{1,2}\left(B, \mathbb{R}^{3}\right)$ is decomposed into $\varphi=\varphi^{\perp}+\varphi^{\top}$, where $\varphi^{\perp}:=\eta(\nu \circ u)$ with $\eta=\varphi \cdot(\nu \circ u) \in$ $L^{\infty}(B, \mathbb{R}) \cap W_{0}^{1,2}(B, \mathbb{R})$. We apply (4.11) to obtain

$$
\delta \mathbf{F}\left(u ; \varphi^{\perp}\right)=\delta \mathbf{F}(u ; \varphi \cdot(\nu \circ u) \nu \circ u)=\int_{u^{-1} \partial A} \varphi \cdot(\nu \circ u) d \lambda .
$$

Moreover, we have $\varphi^{\top} \cdot(\nu \circ u)=0$ almost everywhere on a preimage of a neighborhood of $\partial A$ under $u$, so that $\varphi^{\top}$ and also $-\varphi^{\top}$ are admissible in (4.6), and therefore $\delta \mathbf{F}\left(u ; \varphi^{\top}\right)=0$. We combine this with the corresponding identity for $\varphi^{\top}$ to reach the assertion of (i).

Assertion (iii) is now immediate since in this case $\left(|H \circ u|-\mathcal{H}_{\partial A} \circ u\right)_{+} \equiv 0$ on $u^{-1} \partial A$. This finishes the proof of the lemma.

\section{Existence of Minimizers of the time Discrete VARiational PROBLEM}

We begin by recalling a lemma from [4] that will be crucial for the minimization of the time discrete $H$-functional. The idea is to control a possible bubbling of a minimizing sequence $u_{i}$ by replacing it with a new sequence $\tilde{u}_{i}$ that agrees with the limit map outside of a small set $G$, while on this set, the energy of the $\tilde{u}_{i}$ is 
controlled by the bubble energy (cf. (vi) below). This, in connection with the isoperimetric condition, will enable us to prove the existence of minimizers.

Lemma 5.1. Assume that $u_{i} \rightarrow u$ weakly in $W^{1,2}\left(B, \mathbb{R}^{m}\right)$ and $\left.\left.u_{i}\right|_{\partial B} \rightarrow u\right|_{\partial B}$ uniformly in $L^{\infty}\left(\partial B, \mathbb{R}^{m}\right)$. Then, given $\varepsilon>0$, there exist $R>0$, a measurable set $G \subseteq B$, and maps $\tilde{u}_{i} \in W^{1,2}\left(B, \mathbb{R}^{m}\right)$ such that after passage to a subsequence there holds:

(i) $\tilde{u}_{i}=u$ on $B \backslash G$ with $\mathcal{L}^{2}(G)<\varepsilon$;

(ii) $\left.\tilde{u}_{i}\right|_{\partial B}=\left.u\right|_{\partial B}$ on $\partial B$;

(iii) $\tilde{u}_{i}(x)=u_{i}(x)$ if $\left|u_{i}(x)\right| \geq R$ or $\left|u_{i}(x)-u(x)\right| \geq 1$;

(iv) $\lim _{i \rightarrow \infty}\left\|\tilde{u}_{i}-u_{i}\right\|_{L^{\infty}\left(B, \mathbb{R}^{m}\right)}=0$;

(v) $\tilde{u}_{i} \rightarrow u$ weakly in $W^{1,2}\left(B, \mathbb{R}^{m}\right)$ in the limit $i \rightarrow \infty$;

(vi) $\limsup \sup _{i \rightarrow \infty}\left[\mathbf{D}_{G}\left(\tilde{u}_{i}\right)+\mathbf{D}_{G}(u)\right] \leq \varepsilon+\liminf _{i \rightarrow \infty}\left[\mathbf{D}\left(u_{i}\right)-\mathbf{D}(u)\right]$;

(vii) if the $u_{i}$ takes values in a closed convex subset $A \subseteq \mathbb{R}^{3}$, then the $\tilde{u}_{i}$ can also be chosen to have values in $A$.

The proof of (i) to (vi) was given in [4, Lemma 4.1]. Assertion (vii) is immediate since in [4], the maps $\tilde{u}_{i}$ are constructed as convex combinations of $u_{i}$ and $u$, whose images are contained in the convex set $A$.

We are now prepared to establish the existence of minimizers $u \in W^{1,2}(B, A)$ to the time discrete $H$-functional (4.1) in $\mathcal{S}\left(u_{o} ; A, \sigma\right)$. Here $u_{o} \in W^{1,2}(B, A)$ is a given Dirichlet boundary datum satisfying $(1+\sigma) \mathbf{D}\left(u_{o}\right) \leq s$ with $1<\sigma \leq \infty$, and $z \in W^{1,2}(B, A)$ with $z-u_{o} \in W_{0}^{1,2}\left(B, \mathbb{R}^{3}\right)$ is a fixed second reference surface. The occurrence of $z$ will become clear later in the construction of the time discrete approximations. The class $\mathcal{S}\left(u_{o} ; A, \sigma\right)$ is defined as the class of those maps $u \in$ $W^{1,2}(B, A)$ such that $u-u_{o} \in W_{0}^{1,2}\left(B, \mathbb{R}^{3}\right)$ and $\mathbf{D}(u) \leq \sigma \mathbf{D}\left(u_{o}\right)$.

Theorem 5.2. Suppose that $A \subseteq \mathbb{R}^{3}$ is a closed convex set; the function $H: A \rightarrow \mathbb{R}$ is bounded and continuous and satisfies a spherical isoperimetric condition of type $(c, s)$ on $A$ as stated in (1.5). Moreover, let $u_{o} \in W^{1,2}(B, A)$ be a fixed reference surface for which the inequality $(1+\sigma) \mathbf{D}\left(u_{o}\right) \leq s$ holds for some $1<\sigma \leq \infty$. Finally, let $\mathcal{S}\left(u_{o} ; A, \sigma\right)$ denote the class of all surfaces $\tilde{u} \in \mathcal{S}\left(u_{o} ; A\right)$ with $\mathbf{D}(\tilde{u}) \leq$ $\sigma \mathbf{D}\left(u_{o}\right)$. Then, the following assertion holds: If $\sigma<\infty$ and $c \leq 1$ or if $\sigma=\infty$ and $c<1$, then the variational problem

$$
\mathbf{F}(\tilde{u})=\mathbf{D}(\tilde{u})+2 \mathbf{V}_{H}\left(\tilde{u}, u_{o}\right)+\frac{1}{2 h} \int_{B}|\tilde{u}-z|^{2} d x \longrightarrow \min \quad \text { in } \mathcal{S}\left(u_{o} ; A, \sigma\right)
$$

has a solution.

Proof. We first observe that

$$
\mathbf{M}\left(J_{u}-J_{u_{o}}\right) \leq \mathbf{D}(u)+\mathbf{D}\left(u_{o}\right) \leq(1+\sigma) \mathbf{D}\left(u_{o}\right) \leq s,
$$

for all $u \in \mathcal{S}\left(u_{o} ; A, \sigma\right)$. Therefore $T=J_{u}-J_{u_{o}}$ is a spherical 2-current with spt $T \subseteq A$ and $\mathbf{M}(T) \leq s$. From the spherical isoperimetric condition of type $(c, s)$ on $A$ satisfied by $H$, we infer

$$
2\left|\mathbf{V}_{H}\left(u, u_{o}\right)\right| \leq c \mathbf{M}\left(J_{u}-J_{u_{o}}\right) \leq c\left[\mathbf{D}(u)+\mathbf{D}\left(u_{o}\right)\right]
$$

whenever $u \in \mathcal{S}\left(u_{o} ; A, \sigma\right)$. This implies in particular that

$$
\mathbf{F}(u) \geq \mathbf{D}(u)-2\left|\mathbf{V}_{H}\left(u, u_{o}\right)\right|+\frac{1}{2 h} \int_{B}|u-z|^{2} d x
$$




$$
\geq(1-c) \mathbf{D}(u)-c \mathbf{D}\left(u_{o}\right)+\frac{1}{2 h} \int_{B}|u-z|^{2} d x \geq-c \mathbf{D}\left(u_{o}\right) .
$$

In the last line we used the fact that $c \leq 1$. Therefore, $\mathbf{F}$ is bounded from below on $\mathcal{S}\left(u_{o} ; A, \sigma\right)$ and we can choose a minimizing sequence $\left(u_{i}\right)_{i \in \mathbb{N}}$ in $\mathcal{S}\left(u_{o} ; A, \sigma\right)$, that is,

$$
\lim _{i \rightarrow \infty} \mathbf{F}\left(u_{i}\right)=\inf \left\{\mathbf{F}(u): u \in \mathcal{S}\left(u_{o} ; A, \sigma\right)\right\} .
$$

Moreover, if $\sigma=\infty$ and $c<1$, the last inequality (applied on the minimizing sequence) yields the uniform bound $\sup _{i \in \mathbb{N}} \mathbf{D}\left(u_{i}\right)<\infty$. In the case $\sigma<\infty$ we trivially have $\sup _{i \in \mathbb{N}} \mathbf{D}\left(u_{i}\right) \leq \sigma \mathbf{D}\left(u_{o}\right)<\infty$. In any case (note also that $u_{i}-u_{o} \in$ $\left.W_{0}^{1,2}\left(B, \mathbb{R}^{3}\right)\right)$ the minimizing sequence is a bounded sequence in $W^{1,2}\left(B, \mathbb{R}^{3}\right)$, and therefore after passage to a subsequence we can assume that $u_{i} \rightarrow u$ weakly in $W^{1,2}\left(B, \mathbb{R}^{3}\right)$, strongly in $L^{2}\left(B, \mathbb{R}^{3}\right)$ and a.e. on $B$. For the weak limit $u$ this implies that we have $\mathbf{D}(u) \leq \sigma \mathbf{D}\left(u_{o}\right), u-u_{o} \in W_{0}^{1,2}\left(B, \mathbb{R}^{3}\right)$ and $u(B) \subseteq A$ a.e., that is, $u \in S\left(u_{o} ; A, \sigma\right)$.

We now apply Lemma 5.1 with a given $0<\varepsilon \leq \frac{1}{2} \mathbf{D}(u)$ to obtain, after passing to another subsequence, the maps $\tilde{u}_{i} \in \mathcal{S}\left(u_{o} ; A\right)$ with $\lim _{i \rightarrow \infty}\left\|\tilde{u}_{i}-u_{i}\right\|_{L^{\infty}\left(B, \mathbb{R}^{3}\right)}=0$. Further, by Lemma 3.6 we have that $\mathbf{V}_{H}\left(\tilde{u}_{i}, u_{o}\right)$ and $\mathbf{V}_{H}\left(\tilde{u}_{i}, u_{i}\right)$ are well defined and that

$$
\mathbf{V}_{H}\left(\tilde{u}_{i}, u_{o}\right)-\mathbf{V}_{H}\left(u_{i}, u_{o}\right)=\mathbf{V}_{H}\left(\tilde{u}_{i}, u_{i}\right) \rightarrow 0 \quad \text { as } i \rightarrow \infty .
$$

Next, we observe that (3.1) and Lemma 5.1 (vi) imply for sufficiently large values of $i \in \mathbb{N}$,

$$
\mathbf{M}\left(J_{\tilde{u}_{i}}-J_{u}\right) \leq \mathbf{D}_{G}\left(\tilde{u}_{i}\right)+\mathbf{D}_{G}(u) \leq 2 \varepsilon+\mathbf{D}\left(u_{i}\right)-\mathbf{D}(u) \leq \sigma \mathbf{D}\left(u_{o}\right) \leq s .
$$

Here $G$ is the set given in Lemma 5.1. From the isoperimetric condition and (3.2) we conclude the inequality

$$
2\left|\mathbf{V}_{H}\left(\tilde{u}_{i}, u\right)\right| \leq c \mathbf{M}\left(J_{\tilde{u}_{i}}-J_{u}\right) \leq 2 \varepsilon+\mathbf{D}\left(u_{i}\right)-\mathbf{D}(u),
$$

again for $i$ sufficiently large. Moreover, there holds

$$
\mathbf{V}_{H}\left(\tilde{u}_{i}, u_{o}\right)=\mathbf{V}_{H}\left(\tilde{u}_{i}, u\right)+\mathbf{V}_{H}\left(u, u_{o}\right) .
$$

This allows us to compute

$$
\begin{aligned}
\mathbf{F}\left(u_{i}\right)= & \mathbf{D}\left(u_{i}\right)+2 \mathbf{V}_{H}\left(u_{i}, u_{o}\right)+\frac{1}{2 h} \int_{B}\left|u_{i}-z\right|^{2} d x \\
= & \mathbf{F}(u)+\mathbf{D}\left(u_{i}\right)-\mathbf{D}(u)+2 \mathbf{V}_{H}\left(u_{i}, u_{o}\right)-2 \mathbf{V}_{H}\left(u, u_{o}\right) \\
& +\frac{1}{2 h} \int_{B}\left|u_{i}-z\right|^{2} d x-\frac{1}{2 h} \int_{B}|u-z|^{2} d x \\
= & \mathbf{F}(u)+\mathbf{D}\left(u_{i}\right)-\mathbf{D}(u)+2 \mathbf{V}_{H}\left(\tilde{u}_{i}, u\right)-2 \mathbf{V}_{H}\left(\tilde{u}_{i}, u_{i}\right) \\
& +\frac{1}{2 h} \int_{B}\left|u_{i}-z\right|^{2} d x-\frac{1}{2 h} \int_{B}|u-z|^{2} d x \\
\geq & \mathbf{F}(u)-2 \varepsilon-2 \mathbf{V}_{H}\left(\tilde{u}_{i}, u_{i}\right) \\
& +\frac{1}{2 h} \int_{B}\left|u_{i}-z\right|^{2} d x-\frac{1}{2 h} \int_{B}|u-z|^{2} d x \\
\geq & \mathbf{F}(u)-3 \varepsilon .
\end{aligned}
$$


Here we used in turn (5.2), (5.4), (5.3), once again (5.2) and the strong convergence of $u_{i} \rightarrow u$ in $L^{2}\left(B, \mathbb{R}^{3}\right)$. Since $\varepsilon>0$ was chosen arbitrarily, this finally proves that $u$ minimizes $\mathbf{F}$ in $\mathcal{S}\left(u_{o} ; A, \sigma\right)$.

\section{REgularity OF MINIMIZERS}

In this section we establish the regularity of minimizers $u$ of the variational problem (5.1). To prove the continuity we employ an old device of Morrey [24] that is essentially based on the harmonic replacement on small balls and the comparison of energies.

Theorem 6.1. Suppose that $A, H$ and $u_{o}$ satisfy the conditions of Theorem 5.2 with parameters $\sigma, s$ and $c$. Then, every solution $u$ of (5.1) is Hölder continuous in B. Furthermore, if $\left.u\right|_{\partial B} \in C^{0}\left(\partial B, \mathbb{R}^{3}\right)$, then $u \in C^{0}\left(\bar{B}, \mathbb{R}^{3}\right)$.

Proof. We fix $x_{o} \in B$ and write $u_{r}(\omega):=u(r, \omega):=u\left(x_{o}+r e^{i \omega}\right)$ for $\omega \in[0,2 \pi]$ and $0 \leq r \leq 1-\left|x_{o}\right|$. Then, the function

$$
\left[0,1-\left|x_{o}\right|\right] \ni r \mapsto \Phi(r):=\mathbf{D}_{B_{r}\left(x_{o}\right)}(u)=\frac{1}{2} \int_{0}^{r} \int_{0}^{2 \pi}\left[\left|\frac{\partial u}{\partial \varrho}\right|^{2}+\frac{1}{\varrho^{2}}\left|\frac{\partial u}{\partial \omega}\right|^{2}\right] \varrho d \omega d \varrho
$$

is absolutely continuous, and therefore for almost every $r \in\left[0,1-\left|x_{o}\right|\right]$ we have

$$
\Psi(r):=\frac{1}{2} \int_{0}^{2 \pi}\left|\frac{\partial u_{r}}{\partial \omega}\right|^{2} d \omega \leq r \Phi^{\prime}(r) .
$$

From now on we consider only such $r$ for which (6.1) holds. We define $w \in$ $W^{1,2}\left(B_{r}\left(x_{o}\right), \mathbb{R}^{3}\right)$ to be the harmonic extension to $B_{r}\left(x_{o}\right)$ of the Dirichlet boundary values $\left.u\right|_{\partial B_{r}\left(x_{o}\right)}$. We note that since $w\left(\partial B_{r}\left(x_{0}\right)\right)$ is contained in the convex set $A$ and $w$ is harmonic, there also holds $w\left(B_{r}\left(x_{o}\right)\right) \subset A$. Hence, we have

$$
w \in W^{1,2}\left(B_{r}\left(x_{o}\right), A\right), \quad w-\left.u\right|_{B_{r}\left(x_{o}\right)} \in W_{0}^{1,2}\left(B_{r}\left(x_{o}\right), \mathbb{R}^{3}\right) .
$$

We estimate the Dirichlet integral of the harmonic function $w$ in terms of the tangential derivative over the boundary circle, that is,

$$
\mathbf{D}_{B_{r}\left(x_{o}\right)}(w) \leq \frac{1}{2} \int_{0}^{2 \pi}\left|\frac{\partial u_{r}}{\partial \omega}\right|^{2} d \omega=\Psi(r) .
$$

Now, we define the comparison map

$$
\tilde{u}:= \begin{cases}u & \text { on } B \backslash B_{r}\left(x_{o}\right), \\ w & \text { on } B_{r}\left(x_{o}\right)\end{cases}
$$

We note that $\tilde{u} \in W^{1,2}(B, A)$ and $\tilde{u}-u_{o} \in W_{0}^{1,2}\left(B, \mathbb{R}^{3}\right)$. Moreover, since the harmonic function $w$ is energy-minimizing, we conclude

$$
\mathbf{D}(\tilde{u})=\mathbf{D}_{B \backslash B_{r}\left(x_{o}\right)}(u)+\mathbf{D}_{B_{r}\left(x_{0}\right)}(w) \leq \mathbf{D}(u) \leq \sigma \mathbf{D}\left(u_{0}\right) .
$$

Consequently, we are allowed to take $\tilde{u}$ as a comparison surface. This yields $\mathbf{F}(u) \leq$ $\mathbf{F}(\tilde{u})$ or, equivalently,

$$
\begin{aligned}
\mathbf{D}_{B_{r}\left(x_{o}\right)}(u) \leq & \mathbf{D}_{B_{r}\left(x_{o}\right)}(w)+2\left[\mathbf{V}_{H}\left(\tilde{u}, u_{o}\right)-\mathbf{V}_{H}\left(u, u_{o}\right)\right] \\
& +\frac{1}{2 h} \int_{B_{r}\left(x_{o}\right)}|w-z|^{2} d x-\frac{1}{2 h} \int_{B_{r}\left(x_{o}\right)}|u-z|^{2} d x .
\end{aligned}
$$


Using $\mathbf{V}_{H}(\tilde{u}, u)=\mathbf{V}_{H}\left(\tilde{u}, u_{o}\right)-\mathbf{V}_{H}\left(u, u_{o}\right)$ this can be rewritten as

$$
\begin{aligned}
\mathbf{D}_{B_{r}\left(x_{o}\right)}(u) \leq & \mathbf{D}_{B_{r}\left(x_{o}\right)}(w)+2 \mathbf{V}_{H}(\tilde{u}, u) \\
& +\frac{1}{2 h} \int_{B_{r}\left(x_{o}\right)}|w-z|^{2} d x-\frac{1}{2 h} \int_{B_{r}\left(x_{o}\right)}|u-z|^{2} d x .
\end{aligned}
$$

We now consider the spherical 2-current $J_{\tilde{u}}-J_{u}$ whose mass can be estimated as follows:

$$
\mathbf{M}\left(J_{\tilde{u}}-J_{u}\right) \leq \mathbf{D}_{B_{r}\left(x_{o}\right)}(u)+\mathbf{D}_{B_{r}\left(x_{o}\right)}(w) \leq 2 \mathbf{D}_{B_{r}\left(x_{o}\right)}(u) .
$$

Since $\mathbf{D}_{B_{r}\left(x_{o}\right)}(u) \downarrow 0$ when $r \downarrow 0$, we can choose $r_{o}=r_{o}(s)$ such that

$$
\mathbf{D}_{B_{r}\left(x_{o}\right)}(u) \leq \frac{1}{2} s
$$

so that $\mathbf{M}\left(J_{\tilde{u}}-J_{u}\right) \leq s$ for any $0<r \leq r_{o}$. The volume term can thus be estimated by the spherical isoperimetric condition of type $(c, s)$. This implies

$$
\begin{aligned}
\mathbf{D}_{B_{r}\left(x_{o}\right)}(u) \leq & \mathbf{D}_{B_{r}\left(x_{o}\right)}(w)+c\left[\mathbf{D}_{B_{r}\left(x_{o}\right)}(w)+\mathbf{D}_{B_{r}\left(x_{o}\right)}(u)\right] \\
& +\frac{1}{2 h} \int_{B_{r}\left(x_{o}\right)}|w-z|^{2} d x-\frac{1}{2 h} \int_{B_{r}\left(x_{o}\right)}|u-z|^{2} d x .
\end{aligned}
$$

Since $c<1$ we can reabsorb the term $c \mathbf{D}_{B_{r}\left(x_{o}\right)}(u)$ on the left and obtain

$$
\begin{aligned}
(1-c) & \mathbf{D}_{B_{r}\left(x_{o}\right)}(u) \\
& \leq(1+c) \mathbf{D}_{B_{r}\left(x_{o}\right)}(w)+\frac{1}{2 h} \int_{B_{r}\left(x_{o}\right)}|w-z|^{2} d x-\frac{1}{2 h} \int_{B_{r}\left(x_{o}\right)}|u-z|^{2} d x \\
& =(1+c) \mathbf{D}_{B_{r}\left(x_{o}\right)}(w)+\frac{1}{2 h} \int_{B_{r}\left(x_{o}\right)}|w-u|^{2} d x+\frac{1}{h} \int_{B_{r}\left(x_{o}\right)}(w-u) \cdot(u-z) d x \\
& =(1+c) \mathbf{D}_{B_{r}\left(x_{o}\right)}(w)+I+I I .
\end{aligned}
$$

By the Poincaré inequality (recall (6.2)) we have

$$
I \leq \frac{r^{2}}{2 h} \int_{B_{r}\left(x_{o}\right)}|D w-D u|^{2} d x \leq \frac{2 r}{h}\left[\mathbf{D}_{B_{r}\left(x_{o}\right)}(u)+\mathbf{D}_{B_{r}\left(x_{o}\right)}(w)\right] .
$$

In the last line we used $r \leq 1$. Similarly, we deduce

$$
\begin{aligned}
|I I| & \leq \frac{1}{h}\left(\int_{B_{r}\left(x_{o}\right)}|w-u|^{2} d x\right)^{\frac{1}{2}}\left(\int_{B_{r}\left(x_{o}\right)}|u-z|^{2} d x\right)^{\frac{1}{2}} \\
& \leq \frac{r}{h}\left(\int_{B_{r}\left(x_{o}\right)}|D w-D u|^{2} d x\right)^{\frac{1}{2}}\left(\int_{B_{r}\left(x_{o}\right)}|u-z|^{2} d x\right)^{\frac{1}{2}} \\
& \leq \frac{2 r}{h}\left[\mathbf{D}_{B_{r}\left(x_{o}\right)}(u)+\mathbf{D}_{B_{r}\left(x_{o}\right)}(w)\right]^{\frac{1}{2}}\left(\int_{B_{r}\left(x_{o}\right)}|u-z|^{2} d x\right)^{\frac{1}{2}} \\
& \leq \frac{r}{h}\left[\mathbf{D}_{B_{r}\left(x_{o}\right)}(u)+\mathbf{D}_{B_{r}\left(x_{o}\right)}(w)\right]+\frac{r}{h} \int_{B_{r}\left(x_{o}\right)}|u-z|^{2} d x .
\end{aligned}
$$

Inserting this above and reabsorbing the term $\frac{3 r}{h} \mathbf{D}_{B_{r}\left(x_{o}\right)}(u)$ on the left-hand side, we arrive at

$$
\left(1-c-\frac{3 r}{h}\right) \mathbf{D}_{B_{r}\left(x_{o}\right)}(u) \leq\left(1+c+\frac{3 r}{h}\right) \mathbf{D}_{B_{r}\left(x_{o}\right)}(w)+\frac{r}{h} \int_{B_{r}\left(x_{o}\right)}|u-z|^{2} d x .
$$


We choose $\frac{3 r}{h} \leq \frac{1}{2}(1-c)$, that is $r \leq \frac{1-c}{6} h$, which is possible since $c<1$, and obtain

$$
\mathbf{D}_{B_{r}\left(x_{o}\right)}(u) \leq \frac{3+c}{1-c} \mathbf{D}_{B_{r}\left(x_{o}\right)}(w)+\frac{2 r}{(1-c) h} \int_{B_{r}\left(x_{o}\right)}|u-z|^{2} d x,
$$

as long as $0<r \leq r_{1}:=\min \left\{1-\left|x_{o}\right|, r_{o}, \frac{1-c}{6} h\right\}$. Now, let $K>0$ such that

$$
\frac{1}{2 h} \int_{B}|u-z|^{2} d x \leq K
$$

and moreover $m_{1}:=\frac{3+c}{1-c}$ and $m_{2}:=\frac{4 K}{1-c}$. Then (6.7) and (6.3) yield

$$
\begin{aligned}
\Phi(r) & =\mathbf{D}_{B_{r}\left(x_{o}\right)}(u) \leq m_{1} \mathbf{D}_{B_{r}\left(x_{o}\right)}(w)+m_{2} r \\
& \leq m_{1} \Psi(r)+m_{2} r \leq m_{1} r \Phi^{\prime}(r)+m_{2} r,
\end{aligned}
$$

for any $0<r \leq r_{1}$ for which (6.1) holds, i.e. for almost every $0<r \ll 1$. The preceding differential inequality can be integrated as follows: First we compute

$$
\frac{d}{d \tau}\left(\frac{\Phi(\tau)}{\tau^{\frac{1}{m_{1}}}}\right)=\frac{\Phi^{\prime}(\tau)}{\tau^{\frac{1}{m_{1}}}}-\frac{\Phi(\tau)}{m_{1} \tau^{\frac{1}{m_{1}}+1}}=\frac{1}{\tau^{\frac{1}{m_{1}}}}\left[\Phi^{\prime}(\tau)-\frac{\Phi(\tau)}{m_{1} \tau}\right] \geq-\frac{m_{2}}{m_{1} \tau^{\frac{1}{m_{1}}}},
$$

where in the last step we used (6.8). Integrating this over $[\varrho, r] \subseteq\left(0, r_{1}\right]$, we obtain

$$
\frac{\Phi(r)}{r^{\frac{1}{m_{1}}}}-\frac{\Phi(\varrho)}{\varrho^{\frac{1}{m_{1}}}}+\frac{m_{2}}{m_{1}-1}\left[r^{1-\frac{1}{m_{1}}}-\varrho^{1-\frac{1}{m_{1}}}\right] \geq 0 .
$$

Multiplying this by $\varrho^{\frac{1}{m_{1}}}$, we find

$$
\begin{aligned}
\frac{1}{2} \int_{B_{\varrho}\left(x_{o}\right)}|D u|^{2} d x & =\Phi(\varrho) \leq\left(\frac{\varrho}{r}\right)^{\frac{1}{m_{1}}} \Phi(r)+\frac{m_{2}}{m_{1}-1}\left[r\left(\frac{\varrho}{r}\right)^{\frac{1}{m_{1}}}-\varrho\right] \\
& \leq\left(\frac{\varrho}{r}\right)^{\frac{1}{m_{1}}}\left[\frac{1}{2} \int_{B}|D u|^{2} d x+\frac{m_{2}}{m_{1}-1}\right],
\end{aligned}
$$

whenever $0<\varrho<r \leq r_{1}$. Hence, $u \in C_{\text {loc }}^{0, \alpha}\left(B, \mathbb{R}^{3}\right)$ with $\alpha:=\frac{1}{2 m_{1}}$. Finally, if $\left.u\right|_{\partial B} \in C^{0}\left(\partial B, \mathbb{R}^{3}\right)$, then [21, Lemma 3] yields the desired continuity of $u$ on $\bar{B}$.

\section{A PRIORI ESTIMATES}

Throughout the section we consider a weak solution $u \in W^{1,2}(B, A) \cap C_{\mathrm{loc}}^{0, \alpha}(B, A)$ of the time discretized system

$$
\frac{u-z}{h}-\Delta u+2(H \circ u) D_{1} u \times D_{2} u=\lambda \quad \text { in } B,
$$

where $H \in L^{\infty}(A, \mathbb{R})$ and $h>0$ are given, and where $\lambda$ is a non-negative Radon measure satisfying (4.8); that is,

$$
\lambda \leq \mathcal{L}^{2}\left\llcorner\left(|H \circ u|-\mathcal{H}_{\partial A} \circ u\right)_{+}|D u|^{2} \quad \text { on } u^{-1} \partial A .\right.
$$

Moreover, we assume $z \in W^{1,2}(B, A)$, which implies in particular $z \in L^{q}\left(B, \mathbb{R}^{3}\right)$ for every $q \geq 1$ by Sobolev embedding. We note that in order to have (17.1) for a minimizer of the functional $\mathbf{F}$ from (4.1), we need to assume that $A$ is a convex subset of $\mathbb{R}^{3}$. At this stage the special structure of the non-linearity no longer plays a role, and therefore we consider more general systems of the type

$$
-\Delta u=F \quad \text { in } B
$$


for a right-hand side $F: B \rightarrow \mathbb{R}$ satisfying the growth condition

$$
|F| \leq C_{1}\left(|D u|^{2}+\left|\frac{u-z}{h}\right|\right) \quad \text { in } B
$$

for a given constant $C_{1}>0$. In the case of (7.1) the constant $C_{1}$ depends only on $\|H\|_{L^{\infty}}$ and $\left\|\mathcal{H}_{\partial A}\right\|_{L^{\infty}}$.

In the following two lemmata, we prove the qualitative $C^{1, \gamma}$-regularity of Hölder continuous solutions. To this end, we use the Hölder continuity of the solution in order to deal with the critical growth of the right-hand side and apply classical elliptic bootstrap methods (cf. [12, Thm. III.2.2]). More precisely, for the implementation of these methods we follow the lines of [11, Prop. 7.1], where a related higher order problem is considered. However, the resulting estimates may depend on the solution itself, namely on its $C^{0, \alpha}$-seminorm, which makes the bounds unsuitable for our purposes. Therefore, we derive finer estimates in Lemma 7.3 below, for which the qualitative $C^{1, \gamma}$-regularity is crucial.

We start with the following Morrey-type estimate for the gradient of a solution.

Lemma 7.1. Let $u \in W^{1,2}\left(B_{R}, \mathbb{R}^{3}\right) \cap C^{0, \alpha}\left(\bar{B}_{R}, \mathbb{R}^{3}\right)$ be a weak solution of (7.2), where the right-hand side $F$ satisfies (7.3). Then for any $\beta \in(0, \alpha)$ there exists a constant $C=C\left(C_{1}, C_{2}, C_{3}, \alpha, \beta\right)$ with

$$
C_{2} \equiv[u]_{C^{0, \alpha}\left(B_{R}\right)} \quad \text { and } \quad C_{3} \equiv\left(\int_{B_{R}}\left|\frac{u-z}{h}\right|^{2} d x\right)^{\frac{1}{2}}
$$

such that for any $y \in B_{R}$ there holds the decay estimate

$$
\int_{B_{\varrho}(y)}|D u|^{2} d x \leq C\left(\frac{\varrho}{R_{y}}\right)^{2 \beta}\left[\int_{B_{R_{y}}(y)}|D u|^{2} d x+1\right],
$$

whenever $0<\varrho \leq R_{y}:=R-|y|$.

Proof. For a given ball $B_{r}(y) \subseteq B_{R}$ we test the weak formulation of (7.2) with $\varphi=$ $\eta^{2}\left(u-u_{y, r}\right)$, where $\eta \in C_{0}^{1}\left(B_{r}(y),[0,1]\right)$ fulfills $\eta \equiv 1$ on $B_{r / 2}(y)$ and $|\nabla \eta| \leq 4 / r$. This leads to

$$
\begin{aligned}
\int_{B_{r}(y)}|D u|^{2} \eta^{2} d x & =-2 \int_{B_{r}(y)} D u \cdot \eta \nabla \eta \otimes\left(u-u_{y, r}\right) d x+\int_{B_{r}(y)} F \cdot \eta^{2}\left(u-u_{y, r}\right) d x \\
& =: I+I) \quad I I,
\end{aligned}
$$

with the obvious meaning of $I$ and $I I$. Before starting with the estimation of the right-hand side terms we observe that

$$
\left|u(x)-u_{y, r}\right| \leq C_{2}(2 r)^{\alpha} \leq 2 C_{2} r^{\alpha} \quad \forall x \in B_{r}(y),
$$

by the definition of $C_{2} \equiv[u]_{C^{0, \alpha}\left(B_{R}\right)}$. To estimate $I$ we use Young's inequality to obtain

$$
\begin{aligned}
|I| & \leq \frac{1}{2} \int_{B_{r}(y)}|D u|^{2} \eta^{2} d x+2 \int_{B_{r}(y)}|\nabla \eta|^{2}\left|u-u_{y, r}\right|^{2} d x \\
& \leq \frac{1}{2} \int_{B_{r}(y)}|D u|^{2} \eta^{2} d x+\frac{32}{r^{2}} \int_{B_{r}(y)}\left|u-u_{y, r}\right|^{2} d x \\
& \leq \frac{1}{2} \int_{B_{r}(y)}|D u|^{2} \eta^{2} d x+128 \pi C_{2}^{2} r^{2 \alpha} .
\end{aligned}
$$


The term $I I$ is estimated by (7.3) and again by the Hölder continuity assumption.

This gives

$$
\begin{aligned}
|I I| & \leq 2 C_{1} C_{2} r^{\alpha} \int_{B_{r}(y)}|D u|^{2} \eta^{2}+\left|\frac{u-z}{h}\right| \eta^{2} d x \\
& \leq 2 C_{1} C_{2} r^{\alpha} \int_{B_{r}(y)}|D u|^{2} \eta^{2} d x+2 C_{1} C_{2} r^{\alpha}\left|B_{r}\right|^{\frac{1}{2}}\left(\int_{B_{R}}\left|\frac{u-z}{h}\right|^{2} d x\right)^{\frac{1}{2}} .
\end{aligned}
$$

Keeping in mind the definition of $C_{3}$, we arrive at the bound

$$
|I I| \leq 2 C_{1} C_{2} r^{\alpha} \int_{B_{r}(y)}|D u|^{2} \eta^{2} d x+2 \sqrt{\pi} C_{1} C_{2} C_{3} r^{1+\alpha} .
$$

Joining the estimates for $I$ and $I I$ with (7.4), absorbing the term $\frac{1}{2} \int_{B_{r}(y)}|D u|^{2} \eta^{2} d x$ on the left-hand side and, finally, taking into account the choice of $\eta$, we arrive at

$$
\begin{aligned}
\int_{B_{r / 2}(y)}|D u|^{2} d x & \leq 4 C_{1} C_{2} r^{\alpha} \int_{B_{r}(y)}|D u|^{2} d x+256 \pi C_{2}^{2} r^{2 \alpha}+4 \sqrt{\pi} C_{1} C_{2} C_{3} r^{1+\alpha} \\
& \leq 4 C_{1} C_{2} r^{\alpha} \int_{B_{r}(y)}|D u|^{2} d x+C_{4} r^{2 \alpha} .
\end{aligned}
$$

In the last line we have used $r \leq 1$ and abbreviated $C_{4}:=256 \pi C_{2}^{2}+4 \sqrt{\pi} C_{1} C_{2} C_{3}$. At this stage we take $\beta \in(0, \alpha)$ and choose $R_{o}$ small enough to have

$$
4 C_{1} C_{2} R_{o}^{\alpha} \leq\left(\frac{1}{2}\right)^{2 \beta} .
$$

Note that this fixes $R_{o}$ in dependence of $C_{1}, C_{2}, \alpha$ and $\beta$. With $\Phi(\varrho):=\int_{B_{\varrho}(y)}|D u|^{2} d x$ the previous inequality reads

$$
\Phi\left(\frac{r}{2}\right) \leq\left(\frac{1}{2}\right)^{2 \beta} \Phi(r)+C_{4} r^{2 \alpha},
$$

whenever $0<r \leq \min \left\{R_{o}, R_{y}\right\}$ where $R_{y}:=R-|y|$. Next, we iterate the preceding inequality. For any $k \in \mathbb{N}$ this gives

$$
\begin{aligned}
\Phi\left(2^{-k} r\right) & \leq\left(\frac{1}{2}\right)^{2 k \beta} \Phi(r)+C_{4} r^{2 \alpha} \sum_{j=1}^{k} 2^{-2(j-1) \beta} 2^{2(j-k) \alpha} \\
& \leq\left(\frac{1}{2}\right)^{2 k \beta}\left[\Phi(r)+\frac{2^{2 \beta} C_{4} r^{2 \alpha}}{1-2^{2(\beta-\alpha)}}\right] .
\end{aligned}
$$

Now let $0<\varrho<r \leq \min \left\{R_{o}, R_{y}\right\}$ be given. Then there exists $k \in \mathbb{N}_{0}$ such that $2^{-k-1} r<\varrho \leq 2^{-k} r$. Therefore we obtain

$$
\begin{aligned}
\Phi(\varrho) & \leq \Phi\left(2^{-k} r\right) \leq\left(\frac{1}{2}\right)^{2 k \beta}\left[\Phi(r)+\frac{2^{2 \beta} C_{4} r^{2 \alpha}}{1-2^{2(\beta-\alpha)}}\right] \\
& \leq\left(\frac{2 \varrho}{r}\right)^{2 \beta}\left[\Phi(r)+\frac{2^{2 \beta} C_{4} r^{2 \alpha}}{1-2^{2(\beta-\alpha)}}\right] \leq 4\left[\left(\frac{\varrho}{r}\right)^{2 \beta} \Phi(r)+c(\alpha, \beta) C_{4} \varrho^{2 \beta}\right] .
\end{aligned}
$$

Now, we distinguish between two cases. If $R_{y}=\min \left\{R_{o}, R_{y}\right\}$ we obtain

$$
\int_{B_{\varrho}(y)}|D u|^{2} d x \leq 4\left[\left(\frac{\varrho}{R_{y}}\right)^{2 \beta} \int_{B_{R_{y}}(y)}|D u|^{2} d x+c(\alpha, \beta) C_{4} \varrho^{2 \beta}\right],
$$

whenever $0<\varrho \leq R_{y}$. If $R_{o}=\min \left\{R_{o}, R_{y}\right\}$ the decay estimate yields

$$
\int_{B_{\varrho}(y)}|D u|^{2} d x \leq 4\left[\left(\frac{\varrho}{R_{o}}\right)^{2 \beta} \int_{B_{R_{o}}(y)}|D u|^{2} d x+c(\alpha, \beta) C_{4} \varrho^{2 \beta}\right]
$$




$$
\leq 4\left[\left(\frac{R_{y}}{R_{o}}\right)^{2 \beta}\left(\frac{\varrho}{R_{y}}\right)^{2 \beta} \int_{B_{R_{y}}(y)}|D u|^{2} d x+c(\alpha, \beta) C_{4} \varrho^{2 \beta}\right]
$$

if $\varrho<R_{o}$, while for $\varrho \in\left[R_{o}, R_{y}\right]$ we trivially have

$$
\int_{B_{\varrho}(y)}|D u|^{2} d x \leq\left(\frac{\varrho}{R_{y}}\right)^{2 \beta}\left(\frac{R_{y}}{R_{o}}\right)^{2 \beta} \int_{B_{R_{y}}(y)}|D u|^{2} d x .
$$

Combining the preceding inequalities we finally arrive at

$$
\int_{B_{\varrho}(y)}|D u|^{2} d x \leq 4\left[\left(\frac{R_{y}}{R_{o}}\right)^{2 \beta}\left(\frac{\varrho}{R_{y}}\right)^{2 \beta} \int_{B_{R_{y}}(y)}|D u|^{2} d x+c(\alpha, \beta) C_{4} \varrho^{2 \beta}\right],
$$

whenever $0<\varrho \leq R_{y}$ and $R_{o} \leq R_{y}$. Combined with (7.5), this proves the desired decay estimate with a constant $C=C\left(C_{1}, C_{2}, C_{3}, \alpha, \beta\right)$ and finishes the proof.

Next, we will derive $C^{1, \gamma}$-regularity in a qualitative sense. We point out that the corresponding estimates are not useful for our purposes since they depend on $L^{q}$-norms of $\left|\frac{u-z}{h}\right|$ for $q>2$, which might blow up as $h \downarrow 0$.

Lemma 7.2. Let $u \in W^{1,2}\left(B_{R}, \mathbb{R}^{3}\right) \cap C^{0, \alpha}\left(\bar{B}_{R}, \mathbb{R}^{3}\right)$ be a weak solution of (7.2) where the right-hand side $F$ satisfies (7.3). Then, $D u \in C_{\mathrm{loc}}^{0, \gamma}\left(B_{R}, \mathbb{R}^{3 \cdot 2}\right)$ for any $\gamma \in(0,1)$.

Proof. From the decay estimates in Lemma 7.1 we infer $u \in C_{\text {loc }}^{0, \beta}\left(B_{R}\right)$ for any $\beta \in(0, \alpha)$. First, we will successively improve this Hölder exponent. We begin with the case $3 \beta<2$. We denote by $v \in W^{1,2}\left(B_{r}(y), \mathbb{R}^{3}\right) \cap C^{0}\left(\overline{B_{r}(y)}, \mathbb{R}^{3}\right)$ the harmonic extension of the Dirichlet boundary data $\left.u\right|_{\partial B_{r}(y)}$ to the ball $B_{r}(y) \subseteq B_{R}$. Testing (7.2) with $u-v$ on $B_{r}(y)$ and taking into account that $v$ is harmonic, we deduce

$$
\int_{B_{r}(y)}|D u-D v|^{2} d x=\int_{B_{r}(y)} F \cdot(u-v) d x .
$$

Using the maximum principle (note that $v-u(y)$ is harmonic) and the Hölder continuity of $u$ we infer that

$$
\begin{aligned}
\sup _{B_{r}(y)}|u-v| & \leq \sup _{B_{r}(y)}|u-u(y)|+\sup _{B_{r}(y)}|u(y)-v| \\
& \leq \sup _{B_{r}(y)}|u-u(y)|+\sup _{\partial B_{r}(y)}|u(y)-v| \leq 2 C_{2} r^{\alpha},
\end{aligned}
$$

where $C_{2} \equiv[u]_{C^{0, \alpha}\left(B_{R}\right)}$. Inserting this above and using the growth assumption (7.3) on $F$, we find that

$$
\begin{aligned}
\int_{B_{r}(y)}|D u-D v|^{2} d x & \leq C_{1} \int_{B_{r}(y)}|D u|^{2}|u-v|+\left|\frac{u-z}{h}\right||u-v| d x \\
& \leq 2 C_{1} C_{2} r^{\alpha} \int_{B_{r}(y)}|D u|^{2} d x+C_{1} \int_{B_{r}(y)}\left|\frac{u-z}{h}\right||u-v| d x \\
& \leq 2 C C_{1} C_{2} r^{3 \beta} R_{y}^{-2 \beta}\left[\int_{B_{R_{y}}(y)}|D u|^{2} d x+1\right]+I,
\end{aligned}
$$

where in the last line we used the decay estimate from Lemma 7.1 and we abbreviated $R_{y}:=R-|y|$ and

$$
I:=C_{1} \int_{B_{r}(y)}\left|\frac{u-z}{h}\right||u-v| d x .
$$


To estimate $I$ we use in turn the Cauchy-Schwarz, Poincaré and Young inequalities to have

$$
\begin{aligned}
I & \leq C_{1}\left(\int_{B_{r}(y)}|u-v|^{2} d x\right)^{\frac{1}{2}}\left(\int_{B_{R}}\left|\frac{u-z}{h}\right|^{2} d x\right)^{\frac{1}{2}} \\
& \leq C_{1} C_{3} r\left(\int_{B_{r}(y)}|D u-D v|^{2} d x\right)^{\frac{1}{2}} \\
& \leq \frac{1}{2} \int_{B_{r}(y)}|D u-D v|^{2} d x+\frac{1}{2} C_{1}^{2} C_{3}^{2} r^{2} .
\end{aligned}
$$

Joining this with the estimate from above we arrive at the following comparison estimate:

$$
\begin{aligned}
\int_{B_{r}(y)}|D u-D v|^{2} d x & \leq 4 C C_{1} C_{2} r^{3 \beta} R_{y}^{-2 \beta}\left[\int_{B_{R_{y}}(y)}|D u|^{2} d x+1\right]+C_{1}^{2} C_{3}^{2} r^{2} \\
& \leq C r^{3 \beta} R_{y}^{-2 \beta}\left[\int_{B_{R_{y}}(y)}|D u|^{2} d x+1\right],
\end{aligned}
$$

for a constant $C=C\left(C_{1}, C_{2}, C_{3}, \alpha, \beta\right)$. In the last line we used the assumption $3 \beta<2$ and the fact $R_{y} \leq 1$. Since $v$ is harmonic on $B_{r}(y)$ we have

$$
\int_{B_{\varrho}(y)}|D v|^{2} d x \leq C\left(\frac{\varrho}{r}\right)^{2} \int_{B_{r}(y)}|D v|^{2} d x \leq C\left(\frac{\varrho}{r}\right)^{2} \int_{B_{r}(y)}|D u|^{2} d x .
$$

Together with the comparison estimate this yields

$$
\begin{aligned}
\int_{B_{\varrho}(y)}|D u|^{2} d x & \leq 2 \int_{B_{\varrho}(y)}|D v|^{2} d x+2 \int_{B_{r}(y)}|D u-D v|^{2} d x \\
& \leq C\left(\frac{\varrho}{r}\right)^{2} \int_{B_{r}(y)}|D u|^{2} d x+C r^{3 \beta} R_{y}^{-2 \beta}\left[\int_{B_{R_{y}}(y)}|D u|^{2} d x+1\right] .
\end{aligned}
$$

At this stage we apply Lemma 2.1 with $A, \alpha, \beta, \varepsilon, R$ replaced by $C, 2,3 \beta, 0, R_{y}$ (note that $3 \beta<2$ by assumption) and

$$
\phi(t):=\int_{B_{t}(y)}|D u|^{2} d x, \quad B:=C R_{y}^{-2 \beta}\left[\int_{B_{R_{y}}(y)}|D u|^{2} d x+1\right] .
$$

The application of Lemma 2.1 yields

$$
\int_{B_{\varrho}(y)}|D u|^{2} d x \leq C\left[\left(\frac{\varrho}{r}\right)^{3 \beta} \int_{B_{r}(y)}|D u|^{2} d x+\varrho^{3 \beta} R_{y}^{-2 \beta}\left[\int_{B_{R_{y}}(y)}|D u|^{2} d x+1\right]\right],
$$

for all $0<\varrho \leq r \leq R_{y}$ with a constant $C=C\left(C_{1}, C_{2}, C_{3}, \alpha, \beta\right)$. In particular, we deduce

$$
\int_{B_{r}(y)}|D u|^{2} d x \leq C\left(\frac{r}{R_{y}}\right)^{3 \beta}\left[\int_{B_{R_{y}}(y)}|D u|^{2} d x+1\right] .
$$

This inequality can now be used to improve the comparison estimate as follows: Instead of Lemma 7.1 which was used in (7.6), we now take advantage of the preceding inequality in order to bound $\int_{B_{r}(y)}|D u|^{2} d x$. This implies

$$
\int_{B_{r}(y)}|D u-D v|^{2} d x \leq 2 C_{1} C_{2} r^{\alpha} \int_{B_{r}(y)}|D u|^{2} d x+\int_{B_{r}(y)}\left|\frac{u-z}{h}\right||u-v| d x
$$




$$
\leq C r^{4 \beta} R_{y}^{-3 \beta}\left[\int_{B_{R_{y}}(y)}|D u|^{2} d x+1\right]+I .
$$

Now, if $4 \beta<2$, we proceed as before and estimate the term $I$ according to (7.9). In this way we first obtain the improved comparison estimate

$$
\int_{B_{r}(y)}|D u-D v|^{2} d x \leq C r^{4 \beta} R_{y}^{-3 \beta}\left[\int_{B_{R_{y}}(y)}|D u|^{2} d x+1\right],
$$

and second, along the argumentation from before, the improved decay estimate

$$
\int_{B_{r}(y)}|D u|^{2} d x \leq C\left(\frac{r}{R_{y}}\right)^{4 \beta}\left[\int_{B_{R_{y}}(y)}|D u|^{2} d x+1\right] .
$$

This procedure can be continued as long as $\ell \beta<2$ and leads to

$$
\int_{B_{r}(y)}|D u|^{2} d x \leq C\left(\frac{r}{R_{y}}\right)^{\ell \beta}\left[\int_{B_{R_{y}}(y)}|D u|^{2} d x+1\right] .
$$

Now, let $\ell \in \mathbb{N}$ be the largest integer such that $\ell \beta<2$, i.e. $(\ell+1) \beta \geq 2$. In the case $(\ell+1) \beta=2$ we arrive at the improved comparison estimate

$$
\int_{B_{r}(y)}|D u-D v|^{2} d x \leq C r^{2} R_{y}^{-\ell \beta}\left[\int_{B_{R_{y}}(y)}|D u|^{2} d x+1\right],
$$

and this implies the decay estimate

$$
\int_{B_{\varrho}(y)}|D u|^{2} d x \leq C\left(\frac{\varrho}{r}\right)^{2} \int_{B_{r}(y)}|D u|^{2} d x+C r^{2} R_{y}^{-\ell \beta}\left[\int_{B_{R_{y}}(y)}|D u|^{2} d x+1\right] .
$$

Here the application of the iteration lemma is no longer directly possible, since in the language of the lemma we have $\alpha=\beta=2$. Therefore we choose $\mu:=\left(\ell+\frac{1}{2}\right) \beta<2$ and obtain from the previous inequality

$$
\int_{B_{\varrho}(y)}|D u|^{2} d x \leq C\left(\frac{\varrho}{r}\right)^{\mu} \int_{B_{r}(y)}|D u|^{2} d x+C r^{2} R_{y}^{-\ell \beta}\left[\int_{B_{R_{y}}(y)}|D u|^{2} d x+1\right] .
$$

Now, the application of Lemma 2.1 implies

$$
\begin{aligned}
\int_{B_{r}(y)}|D u|^{2} d x & \leq C\left[\left(\frac{r}{R_{y}}\right)^{\mu} \int_{B_{R_{y}}(y)}|D u|^{2} d x+C r^{\mu} R_{y}^{-\ell \beta}\left[\int_{B_{R_{y}}(y)}|D u|^{2} d x+1\right]\right] \\
& \leq C\left(\frac{r}{R_{y}}\right)^{\mu}\left[\int_{B_{R_{y}}(y)}|D u|^{2} d x+1\right] .
\end{aligned}
$$

At this stage we have $\mu+\beta=\left(\ell+\frac{1}{2}\right) \beta+\beta=(\ell+1) \beta+\frac{1}{2} \beta=2+\frac{1}{2} \beta>2$, and we can join this with the case $(\ell+1) \beta>2$ by letting $\mu$ either be $\left(\ell+\frac{1}{2}\right) \beta$ or $\ell \beta$. In both cases we have (7.11) (cf. (17.10) ). Using (7.11) instead of Lemma 7.1, in (7.6) we get

$$
\int_{B_{r}(y)}|D u-D v|^{2} d x \leq C r^{\beta+\mu} R_{y}^{-\mu}\left[\int_{B_{R_{y}}(y)}|D u|^{2} d x+1\right]+I .
$$

The term $I$ can, at this stage, no longer be estimated as in (7.9). Instead we use the fact that $u, z \in L^{q}\left(B_{R}, \mathbb{R}^{3}\right)$ for any $q>1$. At first, we use this fact for $q=4$, which leads to the bound

$$
I \leq C_{1}\left(\int_{B_{r}(y)}|u-v|^{2} d x\right)^{\frac{1}{2}}\left(\int_{B_{r}(y)}\left|\frac{u-z}{h}\right|^{2} d x\right)^{\frac{1}{2}}
$$




$$
\begin{aligned}
& \leq C_{1} r\left(\int_{B_{r}(y)}|D u-D v|^{2} d x\right)^{\frac{1}{2}}\left|B_{r}(y)\right|^{\frac{1}{4}}\left(\int_{B_{R}}\left|\frac{u-z}{h}\right|^{4} d x\right)^{\frac{1}{4}} \\
& \leq C_{1} C_{3}(4) \pi^{\frac{1}{4}} r^{\frac{3}{2}}\left(\int_{B_{r}(y)}|D u-D v|^{2} d x\right)^{\frac{1}{2}} \\
& \leq \frac{1}{4} \int_{B_{r}(y)}|D u-D v|^{2} d x+C_{1}^{2} C_{3}(4)^{2} \sqrt{\pi} r^{3}
\end{aligned}
$$

where we abbreviated

$$
C_{3}(q):=\left(\int_{B_{R}}\left|\frac{u-z}{h}\right|^{q} d x\right)^{\frac{1}{q}} \quad \text { for any } q \in[1, \infty) .
$$

Joining this with (7.12) we deduce

$$
\int_{B_{r}(y)}|D u-D v|^{2} d x \leq C r^{\beta+\mu} R_{y}^{-\mu}\left[\int_{B_{R_{y}}(y)}|D u|^{2} d x+1\right]+C r^{3} .
$$

We observe that $\beta+\mu \in(2,3)$. Therefore from the preceding inequality we easily infer

$$
\int_{B_{r}(y)}|D u-D v|^{2} d x \leq C r^{\beta+\mu} R_{y}^{-\mu}\left[\int_{B_{R_{y}}(y)}|D u|^{2} d x+1\right],
$$

for all $0<r \leq R_{y}$. Now we recall that there holds

$$
\int_{B_{\varrho}(y)}\left|D v-(D v)_{y, \varrho}\right|^{2} d x \leq C\left(\frac{\varrho}{r}\right)^{4} \int_{B_{r}(y)}\left|D v-(D v)_{y, r}\right|^{2} d x
$$

for all $0<\varrho<r<R_{y}$, since $D v$ is a harmonic function. This leads to a Campanatotype decay estimate for $u$. The precise argument is as follows:

$$
\begin{aligned}
\int_{B_{\varrho}(y)} & \left|D u-(D u)_{y, \varrho}\right|^{2} d x \leq \int_{B_{\varrho}(y)}\left|D u-(D v)_{y, \varrho}\right|^{2} d x \\
& \leq 2 \int_{B_{r}(y)}|D u-D v|^{2} d x+2 C\left(\frac{\varrho}{r}\right)^{4} \int_{B_{r}(y)}\left|D v-(D v)_{y, r}\right|^{2} d x \\
& \leq 2(1+2 C) \int_{B_{r}(y)}|D u-D v|^{2} d x+4 C\left(\frac{\varrho}{r}\right)^{4} \int_{B_{r}(y)}\left|D u-(D u)_{y, r}\right|^{2} d x \\
& \leq C\left(\frac{\varrho}{r}\right)^{4} \int_{B_{r}(y)}\left|D u-(D u)_{y, r}\right|^{2} d x+C r^{\beta+\mu} R_{y}^{-\mu}\left[\int_{B_{R_{y}}(y)}|D u|^{2} d x+1\right] .
\end{aligned}
$$

Since $\varrho \mapsto \int_{B_{\varrho}(y)}\left|D u-(D u)_{y, \varrho}\right|^{2} d x$ is non-decreasing and $\beta+\mu \in(2,3)$, the application of the iteration lemma, i.e. Lemma 2.1 is allowed and yields

$$
\begin{aligned}
& \int_{B_{r}(y)}\left|D u-(D u)_{y, r}\right|^{2} d x \leq C\left[\left(\frac{r}{R_{y}}\right)^{\beta+\mu} \int_{B_{R_{y}}(y)}\left|D u-(D u)_{y, R_{y}}\right|^{2} d x\right. \\
&\left.+r^{\beta+\mu} R_{y}^{-\mu}\left[\int_{B_{R_{y}}(y)}|D u|^{2} d x+1\right]\right] \\
& \leq C\left(\frac{r}{R_{y}}\right)^{\beta+\mu}\left[\int_{B_{R_{y}}(y)}|D u|^{2} d x+1\right]
\end{aligned}
$$


whenever $0<r \leq R_{y}$. Writing $\beta+\mu=2+2 \gamma$ with $\gamma:=\frac{1}{2}(\beta+\mu-2)$ we infer the local Hölder continuity of $D u$, i.e. $D u \in C_{\text {loc }}^{0, \gamma}\left(B_{R}, \mathbb{R}^{3 \cdot 2}\right)$. In particular, we have $|D u| \in L_{\mathrm{loc}}^{\infty}\left(B_{R}\right)$.

Without loss of generality we can assume that $|D u| \in L^{\infty}\left(B_{R}\right)$. This fact can be used to improve the Hölder continuity of $D u$. The argument is as follows: In (7.6) we use that $\|D u\|_{L^{\infty}}<\infty$ in order to estimate

$$
\begin{aligned}
\int_{B_{r}(y)}|D u-D v|^{2} d x & \leq C_{1} \int_{B_{r}(y)}|D u|^{2}|u-v| d x+I \\
& \leq C_{1}\|D u\|_{L^{\infty}}^{2} \int_{B_{r}(y)}|u-v| d x+I \\
& \leq C_{1}\|D u\|_{L^{\infty}}^{2} r \int_{B_{r}(y)}|D u-D v| d x+I \\
& \leq \frac{1}{4} \int_{B_{r}(y)}|D u-D v|^{2} d x+C_{1}^{2}\|D u\|_{L^{\infty}}^{4} \pi r^{4}+I .
\end{aligned}
$$

Here we recall the definition of $I$ from (7.8). For a given Hölder exponent $\gamma \in(0,1)$, we now bound the term $I$ by Hölder's inequality with exponents $2, \frac{2}{\gamma}$ and $\frac{2}{1-\gamma}$ and then by Poincaré's and Young's inequalities, with the result

$$
\begin{aligned}
I & \leq C_{1}\left(\int_{B_{r}(y)}|u-v|^{2} d x\right)^{\frac{1}{2}}\left|B_{r}(y)\right|^{\frac{\gamma}{2}}\left(\int_{B_{R}}\left|\frac{u-z}{h}\right|^{\frac{2}{1-\gamma}} d x\right)^{\frac{1-\gamma}{2}} \\
& \leq C_{1} C_{3}\left(\frac{2}{1-\gamma}\right) \pi^{\frac{\gamma}{2}} r^{1+\gamma}\left(\int_{B_{r}(y)}|D u-D v|^{2} d x\right)^{\frac{1}{2}} \\
& \leq \frac{1}{4} \int_{B_{r}(y)}|D u-D v|^{2} d x+C_{1}^{2} C_{3}\left(\frac{2}{1-\gamma}\right)^{2} \pi^{\gamma} r^{2+2 \gamma} .
\end{aligned}
$$

Combining the last two estimates and reabsorbing the term $\frac{1}{2} \int_{B_{r}(y)}|D u-D v|^{2} d x$ on the left, we arrive at the desired improved comparison estimate

$$
\int_{B_{r}(y)}|D u-D v|^{2} d x \leq C(\gamma) r^{2+2 \gamma}
$$

whenever $0<r \leq R_{y}$, where the constant $C(\gamma)$ depends at most on $C_{1}, C_{3}, \alpha, \beta, \gamma$ and $\|D u\|_{L^{\infty}\left(B_{R}\right)}$. For the Campanato-type decay estimate this improvement means

$$
\begin{aligned}
\int_{B_{\varrho}(y)} & \left|D u-(D u)_{y, \varrho}\right|^{2} d x \\
& \leq 2(1+2 C) \int_{B_{r}(y)}|D u-D v|^{2} d x+4 C\left(\frac{\varrho}{r}\right)^{4} \int_{B_{r}(y)}\left|D u-(D u)_{y, r}\right|^{2} d x \\
& \leq C\left(\frac{\varrho}{r}\right)^{4} \int_{B_{r}(y)}\left|D u-(D u)_{y, r}\right|^{2} d x+C(\gamma) r^{2+2 \gamma},
\end{aligned}
$$

where $\gamma \in(0,1)$ was arbitrary. Then Lemma 2.1 implies for any $0<\varrho<r \leq R_{y}$ that

$$
\int_{B_{\varrho}(y)}\left|D u-(D u)_{y, \varrho}\right|^{2} d x \leq C\left[\left(\frac{\varrho}{r}\right)^{2+2 \gamma} \int_{B_{r}(y)}\left|D u-(D u)_{y, r}\right|^{2} d x+\varrho^{2+2 \gamma}\right],
$$


and this finally gives

$$
\int_{B_{r}(y)}\left|D u-(D u)_{y, r}\right|^{2} d x \leq C\left(\frac{r}{R_{y}}\right)^{2+2 \gamma}\left[\int_{B_{R_{y}}(y)}\left|D u-(D u)_{y, R_{y}}\right|^{2} d x+1\right],
$$

whenever $0<r \leq R_{y}$. Now, Campanato's integral characterization of Hölder continuity implies $D u \in C_{\text {loc }}^{0, \gamma}\left(B_{R}, \mathbb{R}^{3 \cdot 2}\right)$. This proves the desired Hölder-continuity of $D u$ for any Hölder exponent $\gamma \in(0,1)$.

The next step is to establish quantitative $W^{2,2}$-regularity, i.e. with a suitable local estimate. The strategy is to use the qualitative $W^{2,2}$-regularity that is a consequence of the preceding lemma and to derive a suitable $W^{2,2}$-bound by controlling the nonlinearity by interpolation between $W^{2,2}$ and $W^{1,2}$ (i.e. by using the Gagliardo-Nirenberg estimate (2.1)). A similar technique has been used by Struwe [32] in the setting of the harmonic map heat flow (see also Moser 25] for a more sophisticated version in higher dimensions).

Lemma 7.3. Let $u \in W^{1,2}\left(B, \mathbb{R}^{3}\right) \cap C_{\mathrm{loc}}^{0, \alpha}\left(B, \mathbb{R}^{3}\right)$ be a weak solution of (7.2), where the right-hand side $F$ satisfies (7.3) and $z \in W^{1,2}\left(B, \mathbb{R}^{3}\right)$. Then there exists $\varepsilon_{o}=\varepsilon_{o}\left(C_{1}\right)$ such that whenever on $B_{r}(y) \subseteq B_{R}$ the smallness condition

$$
\int_{B_{r}(y)}|D u|^{2} d x \leq \varepsilon_{o}
$$

is satisfied, then there holds

$$
\int_{B_{r / 2}(y)}\left|D^{2} u\right|^{2} d x \leq C\left[r^{-2} \int_{B_{r}(y)}|D u|^{2} d x+\int_{B}\left|\frac{u-z}{h}\right|^{2} d x\right],
$$

for a constant $C=C\left(C_{1}\right)$.

This lemma immediately implies

Corollary 7.4. For a weak solution $W^{1,2}\left(B, \mathbb{R}^{3}\right) \cap C_{\mathrm{loc}}^{0, \alpha}\left(B, \mathbb{R}^{3}\right)$ of (17.1), the estimate from the preceding lemma holds with a constant $C=C\left(\|H\|_{L^{\infty}},\left\|\mathcal{H}_{\partial A}\right\|_{L^{\infty}}\right)$.

Proof of Lemma 7.3. Having the qualitative $L_{\mathrm{loc}}^{\infty}$-regularity for $D u$ from Lemma 7.2 at hand, the right-hand side of the system $(7.2)$ is in $L_{\text {loc }}^{2}\left(B, \mathbb{R}^{3}\right)$ so that $u \in$ $W_{\text {loc }}^{2,2}\left(B, \mathbb{R}^{3}\right)$. This allows us to test the weak formulation of (17.2) with the testing function $\varphi=D_{\alpha}\left(\eta^{2} D_{\alpha} u\right)$ for $\alpha=1,2$, where the smooth cut-off function $\eta$ has compact support in $B$. After integration by parts this gives

$$
\begin{aligned}
\int_{B_{r}(y)} & \left|D D_{\alpha} u\right|^{2} \eta^{2} d x \\
= & -2 \int_{B_{r}(y)} D D_{\alpha} u \cdot \eta D \eta \otimes D_{\alpha} u d x-\int_{B_{r}(y)} F \cdot D_{\alpha}\left(\eta^{2} D_{\alpha} u\right) d x \\
= & : I+I I,
\end{aligned}
$$

with the obvious labeling of $I$ and $I I$. The term $I$ is estimated by means of Young's inequality as follows:

$$
|I| \leq \frac{1}{2} \int_{B_{r}(y)}\left|D D_{\alpha} u\right|^{2} \eta^{2} d x+2 \int_{B_{r}(y)}|D \eta|^{2}\left|D_{\alpha} u\right|^{2} d x .
$$


For the term $I I$ we use (7.3) to deduce

$$
|I I| \leq C_{1} \int_{B_{r}(y)}\left(|D u|^{2}+\left|\frac{u-z}{h}\right|\right)\left[\eta^{2}\left|D_{\alpha}^{2} u\right|+2 \eta|D \eta|\left|D_{\alpha} u\right|\right] d x .
$$

Inserting this above and summing with respect to $\alpha=1,2$, we find, after reabsorbing the term $\frac{1}{2} \int_{B_{r}(y)}\left|D^{2} u\right|^{2} \eta^{2} d x$ on the right, the following estimate:

$$
\begin{aligned}
& \int_{B_{r}(y)}\left|D^{2} u\right|^{2} \eta^{2} d x \\
& \leq 4 \int_{B_{r}(y)}|D u|^{2}|D \eta|^{2} d x+4 C_{1} \int_{B_{r}(y)}\left(|D u|^{2}+\left|\frac{u-z}{h}\right|\right) \eta^{2}\left|D^{2} u\right| d x \\
&+8 C_{1} \int_{B_{r}(y)}\left(|D u|^{2}+\left|\frac{u-z}{h}\right|\right) \eta|D \eta||D u| d x \\
& \leq \frac{1}{2} \int_{B_{r}(y)}\left|D^{2} u\right|^{2} \eta^{2} d x \\
&+C\left[\int_{B_{r}(y)}|D u|^{4} \eta^{2} d x+\int_{B_{r}(y)}|D u|^{2}|D \eta|^{2} d x+\int_{B}\left|\frac{u-z}{h}\right|^{2} d x\right]
\end{aligned}
$$

so that

$$
\begin{aligned}
\int_{B_{r}(y)}\left|D^{2} u\right|^{2} \eta^{2} d x & \\
& \leq C\left[\int_{B_{r}(y)}|D u|^{4} \eta^{2} d x+\int_{B_{r}(y)}|D u|^{2}|D \eta|^{2} d x+\int_{B}\left|\frac{u-z}{h}\right|^{2} d x\right],
\end{aligned}
$$

for a constant $C=C\left(C_{1}\right)$. We now take $0<\varrho<r$ and choose $\eta \in C_{0}^{1}\left(B_{r}(y),[0,1]\right)$ such that $\eta \equiv 1$ on $B_{\varrho}(y), \eta \equiv 0$ outside $B_{r}(y)$ and $|D \eta| \leq 2 /(r-\varrho)$. With this choice the preceding inequality implies

$$
\begin{aligned}
\int_{B_{\varrho}(y)} & \left|D^{2} u\right|^{2} d x \\
& \leq C\left[\int_{B_{r}(y)}|D u|^{4} d x+(r-\varrho)^{-2} \int_{B_{r}(y)}|D u|^{2} d x+\int_{B}\left|\frac{u-z}{h}\right|^{2} d x\right] .
\end{aligned}
$$

The first term on the right-hand side can be estimated by an application of (2.1) on $B_{r}(y)$. This yields

$$
\begin{aligned}
\int_{B_{\varrho}(y)}\left|D^{2} u\right|^{2} d x \leq C & {\left[\mathbf{D}_{B_{r}(y)}(u) \int_{B_{r}(y)}\left|D^{2} u\right|^{2} d x\right.} \\
& \left.+\frac{1+\mathbf{D}_{B_{r}(y)}(u)}{(r-\varrho)^{2}} \int_{B_{r}(y)}|D u|^{2} d x+\int_{B}\left|\frac{u-z}{h}\right|^{2} d x\right] \\
\leq & \frac{1}{2} \int_{B_{r}(y)}\left|D^{2} u\right|^{2} d x+\frac{C+1}{(r-\varrho)^{2}} \int_{B_{r}(y)}|D u|^{2} d x+C \int_{B}\left|\frac{u-z}{h}\right|^{2} d x
\end{aligned}
$$

provided the following smallness condition for $\mathbf{D}_{B_{r}(y)}(u)$ is satisfied:

$$
C \mathbf{D}_{B_{r}(y)}(u) \leq \frac{1}{2}
$$


We note that this is implied by (7.13) if $C \varepsilon_{o} \leq 1$. With the standard iteration Lemma 2.2, we therefore conclude that

$$
\int_{B_{r / 2}(y)}\left|D^{2} u\right|^{2} d x \leq C\left[r^{-2} \int_{B_{r}(y)}|D u|^{2} d x+\int_{B}\left|\frac{u-z}{h}\right|^{2} d x\right],
$$

for a constant $C=C\left(C_{1}\right)$. This finishes the proof of the lemma.

Next, we apply the $W^{2,2}$-estimate from the preceding lemma in order to derive the following compactness result that is analogous to [25, Lemma 4.1].

Lemma 7.5. We suppose that $A$ is a closed convex set, that $H: A \rightarrow \mathbb{R}$ is a bounded continuous function satisfying a spherical isoperimetric condition of type $(c, s)$, and we consider $h_{k}>0$ for $k \in \mathbb{N}$. Assume that $u_{k} \in \mathcal{S}\left(u_{o} ; A, \sigma\right)$ minimizes the functional

$$
\mathbf{F}_{k}(\tilde{u}):=\mathbf{D}(\tilde{u})+2 \mathbf{V}_{H}\left(\tilde{u}, u_{o}\right)+\frac{1}{2 h_{k}} \int_{B}\left|\tilde{u}-z_{k}\right|^{2} d x
$$

where $z_{k} \in W^{1,2}(B, A)$ for $k \in \mathbb{N}$ with $z_{k}-u_{o} \in W_{0}^{1,2}\left(B, \mathbb{R}^{3}\right)$. Further, assume that $u_{k}$ solves the Euler-Lagrange system

$$
\frac{u_{k}-z_{k}}{h_{k}}-\Delta u_{k}+2\left(H \circ u_{k}\right) D_{1} u_{k} \times D_{2} u_{k}=0 \text { weakly in } B,
$$

that $u_{k} \rightarrow u$ strongly in $L^{2}\left(B, \mathbb{R}^{3}\right)$ and that

$$
\limsup _{k \rightarrow \infty} \int_{B}\left|D u_{k}\right|^{2}+\left|\frac{u_{k}-z_{k}}{h_{k}}\right|^{2} d x<\infty .
$$

Then the following holds:

(i) If furthermore

$$
\frac{u_{k}-z_{k}}{h_{k}} \rightarrow-f \quad \text { weakly in } L_{\mathrm{loc}}^{2}\left(B, \mathbb{R}^{3}\right),
$$

then the limit map u solves the equation

$$
-\Delta u+2(H \circ u) D_{1} u \times D_{2} u=f \quad \text { weakly in } B .
$$

(ii) The non-linear $H$-term converges (even without the assumption (7.17)); that is for every $\varphi \in C_{0}^{\infty}\left(B, \mathbb{R}^{3}\right)$ we have

$$
\int_{B}(H \circ u) D_{1} u \times D_{2} u \cdot \varphi d x=\lim _{k \rightarrow \infty} \int_{B}\left(H \circ u_{k}\right) D_{1} u_{k} \times D_{2} u_{k} \cdot \varphi d x .
$$

Proof. We first prove the claim (i) and therefore assume that (7.17) is valid. We define a sequence of Radon measures $\mu_{k}$ on $B$ as follows:

$$
\mu_{k}:=\mathcal{L}^{2}\left\llcorner\left|D u_{k}\right|^{2} .\right.
$$

Since $\left(u_{k}\right)_{k \in \mathbb{N}}$ is a bounded sequence in $W^{1,2}\left(B, \mathbb{R}^{3}\right)$, we have $\sup _{k \in \mathbb{N}} \mu_{k}(B)<\infty$. Therefore, passing to a subsequence that is not relabeled, we can assume that $\mu_{k} \rightarrow$ $\mu$ in the sense of Radon measures, for a Radon measure $\mu$ on $B$ with $\mu(B)<\infty$. With the constant $\varepsilon_{o}>0$ from Lemma 7.3 we define the singular set by

$$
\Sigma:=\left\{x_{o} \in B: \mu\left(\left\{x_{o}\right\}\right) \geq \varepsilon_{o}\right\} .
$$

We mention that $\operatorname{card}(\Sigma)<\infty$, since $\mu(B)<\infty$. Now, for $x_{o} \in B \backslash \Sigma$ there exists a radius $\varrho_{x_{o}}>0$ such that we have $B_{\varrho_{x_{o}}}\left(x_{o}\right) \subseteq B \backslash \Sigma$ and $\mu\left(\overline{B_{\varrho_{x_{o}}}\left(x_{o}\right)}\right)<\varepsilon_{o}$. Since

$$
\limsup _{k \rightarrow \infty} \mu_{k}\left(B_{\varrho_{x_{o}}}\left(x_{o}\right)\right) \leq \mu\left(\overline{B_{\varrho_{x_{o}}}\left(x_{o}\right)}\right)<\varepsilon_{o},
$$


we can conclude that there exists $k_{o} \in \mathbb{N}$ such that

$$
\mu_{k}\left(B_{\varrho_{x_{o}}}\left(x_{o}\right)\right)=\int_{B_{\varrho_{x_{o}}}\left(x_{o}\right)}\left|D u_{k}\right|^{2} d x<\varepsilon_{o} \quad \text { for } k \geq k_{o} .
$$

Therefore the smallness hypothesis (7.13) of Lemma 7.3 is fulfilled for $u_{k}$ with $k \geq k_{o}$. From (7.14) we obtain for $k \geq k_{o}$ that

$$
\begin{aligned}
\int_{B_{\varrho_{x_{o}} / 2}\left(x_{o}\right)}\left|D^{2} u_{k}\right|^{2} d x & \leq C\left[\varrho_{x_{o}}^{-2} \int_{B_{\varrho_{x_{o}}}\left(x_{o}\right)}\left|D u_{k}\right|^{2} d x+\int_{B}\left|\frac{u_{k}-z_{k}}{h_{k}}\right|^{2} d x\right] \\
& \leq C\left[\varrho_{x_{o}}^{-2} \varepsilon_{o}+\sup _{k \in \mathbb{N}} \int_{B}\left|\frac{u_{k}-z_{k}}{h_{k}}\right|^{2} d x\right]=: C .
\end{aligned}
$$

We note that $C$ is independent of $k$. This implies the uniform bound

$$
\sup _{k \geq k_{o}}\left\|u_{k}\right\|_{W^{2,2}\left(B_{\varrho x_{o} / 2}\left(x_{o}\right), \mathbb{R}^{3}\right)}<\infty .
$$

Hence passing again to a subsequence that is not relabeled, we have $u_{k} \rightarrow u$ weakly in $W^{2,2}\left(B_{\varrho_{x_{o}} / 2}\left(x_{o}\right), \mathbb{R}^{3}\right)$ and strongly in $W^{1, q}\left(B_{\varrho_{x_{o}} / 2}\left(x_{o}\right), \mathbb{R}^{3}\right)$ for any $q \geq 1$ so that $u_{k} \in C^{0, \alpha}\left(\overline{B_{\varrho_{x_{o}} / 2}\left(x_{o}\right)}, \mathbb{R}^{3}\right)$ for any $\alpha \in(0,1)$. Because of the Sobolev embedding $W^{1, q} \hookrightarrow L^{\infty}$ that holds for $q>2$, we have, moreover, $u_{k} \rightarrow u$ uniformly on $B_{\varrho_{x_{o}} / 2}\left(x_{o}\right)$. Hence for any $\varphi \in C_{0}^{\infty}\left(B_{\varrho_{x_{o}} / 2}\left(x_{o}\right), \mathbb{R}^{3}\right)$ we have

$$
\begin{aligned}
& \int_{B_{\varrho_{x_{o}} / 2}\left(x_{o}\right)} D u \cdot D \varphi+2(H \circ u) D_{1} u \times D_{2} u \cdot \varphi-f \cdot \varphi d x \\
& \quad=\lim _{k \rightarrow \infty} \int_{B_{\varrho_{x_{o} / 2}\left(x_{o}\right)} D u_{k} \cdot D \varphi+2\left(H \circ u_{k}\right) D_{1} u_{k} \times D_{2} u_{k} \cdot \varphi+\frac{u_{k}-z_{k}}{h_{k}} \cdot \varphi d x=0 .}
\end{aligned}
$$

Since $x_{o} \in B \backslash \Sigma$ was arbitrary, we deduce by a partition of unity argument that $u$ solves

$$
-\Delta u+2(H \circ u) D_{1} u \times D_{2} u=f \quad \text { weakly in } B \backslash \Sigma .
$$

Next, we wish to establish that the preceding equation holds on the whole of $B$. For this aim we apply a simple capacity argument. Let $\Sigma=\left\{x_{1}, \ldots, x_{m}\right\}$. For a sufficiently small radius $r>0$ the balls $B_{2 r}\left(x_{\ell}\right), \ell=1, \ldots, m$, are pairwise disjoint and contained in $B$. We choose $\eta \in C_{0}^{1}\left(B_{2}(0)\right)$ with $\eta \equiv 1$ on $B_{1}(0), 0 \leq \eta \leq 1$ and $|D \eta| \leq 2$ and set $\eta_{\ell}(x):=\eta\left(\frac{x-x_{\ell}}{r}\right)$. We point out that by the choice of $\eta$, these functions satisfy

$$
\int_{B_{2 r}\left(x_{\ell}\right)}\left|D \eta_{\ell}\right|^{2} d x \leq 16 \pi
$$

For an arbitrary function $\psi \in C_{0}^{\infty}\left(B, \mathbb{R}^{3}\right)$, we choose the testing function

$$
\varphi:=\psi \prod_{\ell=1}^{m}\left(1-\eta_{\ell}\right)
$$

in the weak form of (7.21). Note that $\varphi \equiv 0$ in a neighborhood of $\Sigma$, and therefore $\varphi$ is admissible as a test function. We compute

$$
D \varphi=D \psi \prod_{\ell=1}^{m}\left(1-\eta_{\ell}\right)-\sum_{j=1}^{m} \psi \otimes D \eta_{j} \prod_{\ell \neq j}\left(1-\eta_{\ell}\right) .
$$


Thus, we have

$$
\begin{aligned}
\int_{B} D u \cdot D \varphi d x= & \int_{B} D u \cdot D \psi d x-\int_{B} D u \cdot D \psi\left[1-\prod_{\ell=1}^{m}\left(1-\eta_{\ell}\right)\right] d x \\
& -\sum_{j=1}^{m} \int_{B} D u \cdot \psi \otimes D \eta_{j} \prod_{\ell \neq j}\left(1-\eta_{\ell}\right) d x \\
= & : \int_{B} D u \cdot D \psi d x-I-I I,
\end{aligned}
$$

with the obvious labeling for $I$ and $I I$. Since $\operatorname{spt}\left[1-\prod_{\ell=1}^{m}\left(1-\eta_{\ell}\right)\right] \subseteq \bigcup_{\ell=1}^{m} B_{2 r}\left(x_{\ell}\right)$ and $0 \leq \eta_{\ell} \leq 1$, we have

$$
|I| \leq \sum_{\ell=1}^{m} \int_{B_{2 r}\left(x_{\ell}\right)}\left|D u\|D \psi \mid d x \leq\| D \psi \|_{L^{2}(B)} \sum_{\ell=1}^{m}\left(\int_{B_{2 r}\left(x_{\ell}\right)}|D u|^{2} d x\right)^{\frac{1}{2}} .\right.
$$

Similarly, we deduce, keeping in mind (7.22),

$$
|I I| \leq \sum_{\ell=1}^{m} \int_{B_{2 r}\left(x_{\ell}\right)}\left|D u\left\|\psi|| D \eta_{\ell} \mid d x \leq 4 \sqrt{\pi}\right\| \psi \|_{L^{\infty}(B)} \sum_{\ell=1}^{m}\left(\int_{B_{2 r}\left(x_{\ell}\right)}|D u|^{2} d x\right)^{\frac{1}{2}} .\right.
$$

Next, we estimate the term involving the function $H$. We rewrite this term in the form

$$
\begin{aligned}
\int_{B} & (H \circ u) D_{1} u \times D_{2} u \cdot \varphi d x \\
\quad= & \int_{B}(H \circ u) D_{1} u \times D_{2} u \cdot \psi d x-\int_{B}(H \circ u) D_{1} u \times D_{2} u \cdot \psi\left[1-\prod_{\ell=1}^{m}\left(1-\eta_{\ell}\right)\right] d x \\
\quad= & : \int_{B}(H \circ u) D_{1} u \times D_{2} u \cdot \psi d x-I I I .
\end{aligned}
$$

For the term $I I I$ we have

$$
|I I I| \leq \frac{1}{2}\|H\|_{L^{\infty}\left(\mathbb{R}^{3}\right)}\|\psi\|_{L^{\infty}(B)} \sum_{\ell=1}^{m} \int_{B_{2 r}\left(x_{\ell}\right)}|D u|^{2} d x .
$$

Next, we write the term involving $f$ as

$$
-\int_{B} f \cdot \varphi=-\int_{B} f \cdot \psi d x+\int_{B} f \cdot \psi\left[1-\prod_{\ell=1}^{m}\left(1-\eta_{\ell}\right)\right] d x=:-\int_{B} f \cdot \psi d x-I V
$$

and estimate $I V$ by

$$
|I V| \leq \sum_{\ell=1}^{m} \int_{B_{2 r}\left(x_{\ell}\right)}|f||\psi| d x \leq\|\psi\|_{L^{2}(B)} \sum_{\ell=1}^{m}\left(\int_{B_{2 r}\left(x_{\ell}\right)}|f|^{2} d x\right)^{\frac{1}{2}} .
$$

Joining the preceding identities we arrive at

$$
\int_{B}\left[D u \cdot D \psi+2(H \circ u) D_{1} u \times D_{2} u \cdot \psi-f \cdot \psi\right] d x=I+I I+2 I I I+I V,
$$

and since

$$
\lim _{r \downarrow 0}[|I|+|I I|+2|I I I|+|I V|]=0,
$$


we see that there holds

$$
\int_{B}\left[D u \cdot D \psi+2(H \circ u) D_{1} u \times D_{2} u \cdot \psi-f \cdot \psi\right] d x=0,
$$

whenever $\psi \in C_{0}^{\infty}\left(B, \mathbb{R}^{3}\right)$. This holds true (by an approximation argument) for any $\psi \in L^{\infty}\left(B, \mathbb{R}^{3}\right) \cap W_{0}^{1,2}\left(B, \mathbb{R}^{3}\right)$ and proves (7.18) .

Finally, claim (ii) can be obtained as follows: Due to the bound (7.16), by passing to a non-relabeled subsequence we may assume that $u_{k} \rightarrow u$ weakly in $W^{1,2}\left(B, \mathbb{R}^{3}\right)$ and $f_{k}:=\frac{u_{k}-z_{k}}{h_{k}} \rightarrow-f$ weakly in $L^{2}\left(B, \mathbb{R}^{3}\right)$. Therefore we are in a position to apply claim (i) which, together with equation (7.15) for the maps $u_{k}$, implies

$$
\begin{aligned}
\int_{B} 2(H \circ u) D_{1} u \times D_{2} u \cdot \varphi d x & =\int_{B}-D u \cdot D \varphi+f \cdot \varphi d x \\
& =\lim _{k \rightarrow \infty} \int_{B}-D u_{k} \cdot D \varphi-f_{k} \cdot \varphi d x \\
& =\lim _{k \rightarrow \infty} \int_{B} 2\left(H \circ u_{k}\right) D_{1} u_{k} \times D_{2} u_{k} \cdot \varphi d x
\end{aligned}
$$

whenever $\varphi \in L^{\infty}\left(B, \mathbb{R}^{3}\right) \cap W_{0}^{1,2}\left(B, \mathbb{R}^{3}\right)$. Since the left-hand side does not depend on the subsequence, the last equality must hold for the whole sequence. This finishes the proof of the lemma.

\section{The APproximation SCHEME}

In this section we follow a method due to Moser 25] for the construction of solutions to a heat flow by a time discretization approach. Throughout this section, we suppose that the general assumptions listed in Section 1 are in force. We recall that by $\mathcal{S}\left(u_{o} ; A, \sigma\right)$ we denote the class of all surfaces $w \in \mathcal{S}\left(u_{o} ; A\right)$ with $\mathbf{D}(w) \leq$ $\sigma \mathbf{D}\left(u_{o}\right)$. Let $j \in \mathbb{N}_{0}$ and $h>0$ be given. We define sequences of energy functionals $\mathbf{F}_{j, h}$ and maps $u_{j, h} \in \mathcal{S}\left(u_{o} ; A, \sigma\right)$ according to the following recursive iteration scheme: We set $u_{o, h}=u_{o}$. Once $u_{j-1, h}$ is constructed, the map $u_{j, h} \in \mathcal{S}\left(u_{o} ; A, \sigma\right)$ is chosen as a solution of the variational problem

$$
\mathbf{F}_{j, h}(\tilde{u}) \longrightarrow \min \quad \text { in } \mathcal{S}\left(u_{o} ; A, \sigma\right)
$$

where

$$
\mathbf{F}_{j, h}(\tilde{u}):=\mathbf{D}(\tilde{u})+2 \mathbf{V}_{H}\left(\tilde{u}, u_{o}\right)+\frac{1}{2 h} \int_{B}\left|\tilde{u}-u_{j-1, h}\right|^{2} d x .
$$

We note that Theorem 5.2 yields the existence of a minimizer $u_{j, h} \in \mathcal{S}\left(u_{o} ; A, \sigma\right)$. In particular, we have $\mathbf{D}\left(u_{j, h}\right) \leq \sigma \mathbf{D}\left(u_{o}\right)$. The following lemma ensures that we actually have the strict inequality $\mathbf{D}\left(u_{j, h}\right)<\sigma \mathbf{D}\left(u_{o}\right)$ for any $j \in \mathbb{N}$.

Lemma 8.1. Suppose that the assumptions of Theorem 5.2 are in force. If

$$
c \leq \frac{\sigma-1}{\sigma+1} \text { and } \sigma<\infty \quad \text { or } \quad c<1 \text { and } \sigma=\infty,
$$

then for any $j \in \mathbb{N}$ (and any minimizer $u_{j, h} \in \mathcal{S}\left(A ; u_{o}, \sigma\right)$ ) the strict inequality $\mathbf{D}\left(u_{j, h}\right)<\sigma \mathbf{D}\left(u_{o}\right)$ holds.

Proof. Let $j \in \mathbb{N}$ be fixed. Since $u_{j, h}$ is a solution of the variational problem (8.1) and $u_{j-1, h} \in \mathcal{S}\left(u_{o} ; A, \sigma\right)$, we have

$$
\mathbf{F}_{j, h}\left(u_{j, h}\right)=\mathbf{D}\left(u_{j, h}\right)+2 \mathbf{V}_{H}\left(u_{j, h}, u_{o}\right)+\frac{1}{2 h} \int_{B}\left|u_{j, h}-u_{j-1, h}\right|^{2} d x
$$




$$
\leq \mathbf{F}_{j, h}\left(u_{j-1, h}\right)=\mathbf{D}\left(u_{j-1, h}\right)+2 \mathbf{V}_{H}\left(u_{j-1, h}, u_{o}\right)
$$

or, equivalently,

$$
\begin{aligned}
\mathbf{D}\left(u_{j, h}\right) \leq & \mathbf{D}\left(u_{j-1, h}\right)+2\left[\mathbf{V}_{H}\left(u_{j-1, h}, u_{o}\right)-\mathbf{V}_{H}\left(u_{j, h}, u_{o}\right)\right] \\
& -\frac{1}{2 h} \int_{B}\left|u_{j, h}-u_{j-1, h}\right|^{2} d x
\end{aligned}
$$

Iterating this inequality, keeping in mind that $u_{o, h}=u_{o}$ and using the isoperimetric condition, we obtain

$$
\begin{aligned}
\mathbf{D}\left(u_{j, h}\right) \leq & \mathbf{D}\left(u_{o}\right)+\sum_{\ell=1}^{j} 2\left[\mathbf{V}_{H}\left(u_{\ell-1, h}, u_{o}\right)-\mathbf{V}_{H}\left(u_{\ell, h}, u_{o}\right)\right] \\
& -\sum_{\ell=1}^{j} \frac{1}{2 h} \int_{B}\left|u_{\ell, h}-u_{\ell-1, h}\right|^{2} d x \\
= & \mathbf{D}\left(u_{o}\right)-2 \mathbf{V}_{H}\left(u_{j, h}, u_{o}\right)-\sum_{\ell=1}^{j} \frac{1}{2 h} \int_{B}\left|u_{\ell, h}-u_{\ell-1, h}\right|^{2} d x \\
\leq & \mathbf{D}\left(u_{o}\right)+c\left[\mathbf{D}\left(u_{o}\right)+\mathbf{D}\left(u_{j, h}\right)\right]-\sum_{\ell=1}^{j} \frac{1}{2 h} \int_{B}\left|u_{\ell, h}-u_{\ell-1, h}\right|^{2} d x \\
\leq & (1+c(1+\sigma)) \mathbf{D}\left(u_{o}\right)-\sum_{\ell=1}^{j} \frac{1}{2 h} \int_{B}\left|u_{\ell, h}-u_{\ell-1, h}\right|^{2} d x .
\end{aligned}
$$

Now we distinguish between the cases $\sigma<\infty$ and $\sigma=\infty$. In the case $\sigma=\infty$ (and $c<1$ ) there is nothing to show, since $\mathbf{D}\left(u_{j, h}\right)<\infty$. In the case $\sigma<\infty$ the assumption $c \leq \frac{\sigma-1}{\sigma+1}$, together with (8.3), leads to

$$
\mathbf{D}\left(u_{j, h}\right) \leq \sigma \mathbf{D}\left(u_{o}\right)-\sum_{\ell=1}^{j} \frac{1}{2 h} \int_{B}\left|u_{\ell, h}-u_{\ell-1, h}\right|^{2} d x .
$$

Now, suppose that

$$
\int_{B}\left|u_{\ell, h}-u_{\ell-1, h}\right|^{2} d x>0 \quad \text { for some } \ell \in\{1, \ldots, j\} .
$$

Then, we have the strict inequality $\mathbf{D}\left(u_{j, h}\right)<\sigma \mathbf{D}\left(u_{o}\right)$. On the other hand, if

$$
\int_{B}\left|u_{\ell, h}-u_{\ell-1, h}\right|^{2} d x=0 \quad \text { for any } \ell \in\{1, \ldots, j\},
$$

we have $u_{j, h}=u_{j-1, h}=\cdots=u_{1, h}=u_{o}$. Since $\sigma>1$ we again trivially have the strict inequality $\mathbf{D}\left(u_{j, h}\right)=\mathbf{D}\left(u_{o}\right)<\sigma \mathbf{D}\left(u_{o}\right)$. This finishes the proof of the lemma.

Remark 8.2. For later use we rewrite (8.3) (more precisely the second last line) as follows:

$$
(1-c) \mathbf{D}\left(u_{j, h}\right)+\sum_{\ell=1}^{j} \frac{1}{2 h} \int_{B}\left|u_{\ell, h}-u_{\ell-1, h}\right|^{2} d x \leq(1+c) \mathbf{D}\left(u_{o}\right) .
$$


Since $c<1$ this implies

$$
\sum_{\ell=1}^{j} \frac{1}{2 h} \int_{B}\left|u_{\ell, h}-u_{\ell-1, h}\right|^{2} d x \leq 2 \mathbf{D}\left(u_{o}\right),
$$

and also

$$
\mathbf{D}\left(u_{j, h}\right) \leq \frac{1+c}{1-c} \mathbf{D}\left(u_{o}\right)
$$

Now, we are in a position to show that the minimizers $u_{j, h}$ are actually solutions of the Euler-Lagrange system.

Theorem 8.3. Suppose that the assumptions of Theorem 5.2 are in force and that $\partial A$ is of class $C^{2}$ with bounded principal curvatures. Further, assume that (8.2) holds and

$$
|H(a)| \leq \mathcal{H}_{\partial A}(a) \quad \text { for } a \in \partial A .
$$

Then any minimizer $u_{j, h} \in \mathcal{S}\left(A ; u_{o}, \sigma\right)$ with $j \in \mathbb{N}$ satisfies the Euler-Lagrange system weakly on $B$, that is,

$$
\int_{B}\left[\frac{u_{j, h}-u_{j-1, h}}{h} \cdot \varphi+D u_{j, h} \cdot D \varphi+2\left(H \circ u_{j, h}\right) D_{1} u_{j, h} \times D_{2} u_{j, h} \cdot \varphi\right] d x=0
$$

whenever $\varphi \in L^{\infty}\left(B, \mathbb{R}^{3}\right) \cap W_{0}^{1,2}\left(B, \mathbb{R}^{3}\right)$.

Proof. Since assumption (8.2) allows to apply Lemma 8.1, we infer $\mathbf{D}\left(u_{j, h}\right)<$ $\sigma \mathbf{D}\left(u_{o}\right)$, and therefore Lemma 4.3, respectively Lemma 4.4 are applicable as follows: First $u_{j, h}$ satisfies (4.7) with a Radon measure $\lambda_{j, h}$ on the right-hand side which itself satisfies estimate (4.8). Since we are imposing the pointwise curvature condition (8.6) along $\partial A$, Lemma 4.4 (iii) implies $\lambda_{j, h} \equiv 0$, hence $u_{j, h}$ solves the Euler-Lagrange system weakly on $B$. This completes the proof.

We now define the approximating sequence, which will lead to the desired weak solution of our Cauchy-Dirichlet problem (1.1) in the limit $h \downarrow 0$. We let

$$
u_{h}(x, t):=u_{j, h}(x) \text { for } j h \leq t<(j+1) h, j \in \mathbb{N}_{0} \text { and } x \in B .
$$

Recall that we defined $u_{o, h}=u_{o}$, and therefore $u_{h}(\cdot, t)=u_{o}$ for $t \in[0, h)$. Using the finite difference quotient operator in time, that is,

$$
\Delta_{t}^{h} v(x, t):=\frac{v(x, t)-v(x, t-h)}{h},
$$

we can rewrite the Euler-Lagrange system from above in the following form:

$$
\Delta_{t}^{h} u_{h}-\Delta u_{h}+2\left(H \circ u_{h}\right) D_{1} u_{h} \times D_{2} u_{h}=0 \quad \text { in } B \times(h, \infty) .
$$

We mention that $u_{h}=u_{o}$ on $\partial B \times(0, \infty) \cup B \times[0, h)$. Let $T \geq h$. Then there exists $j \in \mathbb{N}$ such that $j h \leq T<(j+1) h$, and therefore

$$
\begin{aligned}
\frac{1}{2} \int_{h}^{T} \int_{B}\left|\Delta_{t}^{h} u_{h}\right|^{2} d x d t & \leq \frac{1}{2} \int_{h}^{(j+1) h} \int_{B}\left|\Delta_{t}^{h} u_{h}\right|^{2} d x d t \\
& =\sum_{\ell=1}^{j} \frac{1}{2} \int_{\ell h}^{(\ell+1) h} \int_{B}\left|\Delta_{t}^{h} u_{h}\right|^{2} d x d t
\end{aligned}
$$




$$
=\sum_{\ell=1}^{j} \frac{1}{2 h} \int_{B}\left|u_{\ell, h}(x)-u_{\ell-1, h}(x)\right|^{2} d x \leq 2 \mathbf{D}\left(u_{o}\right) .
$$

Here we used the uniform bound (8.4) from Remark 8.2. Next, we note that

$$
\mathbf{D}\left(u_{h}(\cdot, T)\right)=\mathbf{D}\left(u_{j, h}\right) \leq C \mathbf{D}\left(u_{o}\right),
$$

which immediately follows from the construction of $u_{j, h}$ with $C=\sigma$ when $\sigma<\infty$, and in the case $\sigma=\infty$ follows (with $C=\frac{1+c}{1-c}$ ) from (8.5) in Remark 8.2. In any case we have

$$
\sup _{h>0} \sup _{T>h}\left[\mathbf{D}\left(u_{h}(\cdot, T)\right)+\frac{1}{2} \int_{h}^{T} \int_{\Omega}\left|\Delta_{t}^{h} u_{h}\right|^{2} d x d t\right] \leq C \mathbf{D}\left(u_{o}\right),
$$

for a constant $C>0$ independent of $T$ and $h>0$. When $0<T \leq h$ we have $u_{h}(x, T)=u_{o, h}=u_{o}$ by the construction of the sequence $\left(u_{j, h}\right)_{j \in \mathbb{N}_{0}}$. Therefore from the preceding inequality we also infer the uniform bound

$$
\sup _{h>0} \sup _{T>0} \mathbf{D}\left(u_{h}(\cdot, T)\right) \leq C \mathbf{D}\left(u_{o}\right) .
$$

Since $u_{h}(\cdot, T)-u_{o} \in W_{0}^{1,2}\left(B, \mathbb{R}^{3}\right)$, an application of Poincaré's inequality implies

$$
\begin{aligned}
\left\|u_{h}(\cdot, T)\right\|_{L^{2}(B)} & \leq\left\|u_{h}(\cdot, T)-u_{o}\right\|_{L^{2}(B)}+\left\|u_{o}\right\|_{L^{2}(B)} \\
& \leq\left\|D u_{h}(\cdot, T)-D u_{o}\right\|_{L^{2}(B)}+\left\|u_{o}\right\|_{L^{2}(B)} \\
& \leq 2\left[\sqrt{\mathbf{D}\left(u_{h}(\cdot, T)\right)}+\sqrt{\mathbf{D}\left(u_{o}\right)}\right]+\left\|u_{o}\right\|_{L^{2}(B)} \\
& \leq 2(1+\sqrt{C}) \sqrt{\mathbf{D}\left(u_{o}\right)}+\left\|u_{o}\right\|_{L^{2}(B)},
\end{aligned}
$$

which, combined with the uniform energy-bound from above, yields the following uniform bound of the $L^{\infty}-W^{1,2}$-norm for $u_{h}$ :

$$
\sup _{h>0}\left\|u_{h}\right\|_{L^{\infty}\left((0, \infty), W^{1,2}\left(B, \mathbb{R}^{3}\right)\right)} \leq C\left\|u_{o}\right\|_{W^{1,2}\left(B, \mathbb{R}^{3}\right)},
$$

again for a constant $C>0$ independent of $T$ and $h>0$.

Next, we wish to establish continuity with respect to the time direction. For fixed times $0 \leq t_{1}<t_{2}$, we find $i \in \mathbb{N}_{0}$ such that $i h \leq t_{1}<(i+1) h$. In the case $i h \leq t_{1}<t_{2}<(i+1) h$ we trivially have $\left\|u_{s, h}\left(\cdot, t_{2}\right)-u_{s, h}\left(\cdot, t_{1}\right)\right\|_{L^{2}(\Omega)}=0$, while in the case $i h \leq t_{1}<(i+1) h \leq t_{2}$ we find $j>i$ such that $i h \leq t_{1}<(i+1) h \leq j h \leq$ $t_{2}<(j+1) h$. We thereby obtain

$$
\begin{aligned}
& \left\|u_{s, h}\left(\cdot, t_{2}\right)-u_{s, h}\left(\cdot, t_{1}\right)\right\|_{L^{2}(\Omega)}=\left\|u_{s, h}^{(j)}-u_{s, h}^{(i)}\right\|_{L^{2}(\Omega)} \\
& \quad \leq \sum_{\ell=i+1}^{j}\left\|u_{s, h}^{(\ell)}-u_{s, h}^{(\ell-1)}\right\|_{L^{2}(\Omega)} \leq\left[\sum_{\ell=i+1}^{j}\left\|u_{s, h}^{(\ell)}-u_{s, h}^{(\ell-1)}\right\|_{L^{2}(\Omega)}^{2}\right]^{\frac{1}{2}} \sqrt{j-i} \\
& \quad \leq 2 \sqrt{\mathbf{D}\left(u_{o}\right)}\left[\sqrt{t_{2}-t_{1}}+\sqrt{h}\right] .
\end{aligned}
$$

In the last line we used the bound (8.3) and the fact that $h(j-i)=h(j-(i+1))+h \leq$ $t_{2}-t_{1}+h$. We conclude that the preceding inequality holds for any $h>0$ and $0 \leq t_{1}<t_{2}$. As in [5, Lemma 4.1] we deduce from [28, Theorem 3] that there exist sequences $h_{i} \downarrow 0,0<s_{i} \rightarrow s \in[0,1]$ and a map

$$
u \in C^{0, \frac{1}{2}}\left([0, \infty) ; L^{2}\left(B, \mathbb{R}^{3}\right)\right) \cap L^{\infty}\left([0, \infty) ; W^{1,2}\left(B, \mathbb{R}^{3}\right)\right)
$$

such that

$$
u_{h_{i}} \rightarrow u \quad \text { in } C^{0}\left([0, T] ; L^{2}\left(B, \mathbb{R}^{3}\right)\right) \text { as } i \rightarrow \infty, \text { for all } T>0 .
$$


Moreover, we can achieve

$$
D u_{h_{i}} \rightarrow D u \quad \text { in } L^{2}\left(B \times(0, \infty), \mathbb{R}^{3 \cdot 2}\right) \text { as } i \rightarrow \infty .
$$

Now, for $\varphi \in C_{0}^{\infty}\left(B \times(0, \infty), \mathbb{R}^{3}\right)$ we have

$$
\begin{aligned}
\int_{0}^{\infty} \int_{B} u \cdot \partial_{t} \varphi d x d t & =\lim _{i \rightarrow \infty} \int_{0}^{\infty} \int_{B} u_{h_{i}} \cdot \Delta_{t}^{-h_{i}} \varphi d x d t \\
& =-\lim _{i \rightarrow \infty} \int_{h_{i}}^{\infty} \int_{B} \Delta_{t}^{h_{i}} u_{h_{i}} \cdot \varphi d x d t \\
& \leq\|\varphi\|_{L^{2}(B \times(0, \infty))} \limsup _{i \rightarrow \infty}\left(\int_{\operatorname{spt} \varphi}\left|\Delta_{t}^{h_{i}} u_{h_{i}}\right|^{2} d x\right)^{\frac{1}{2}} \\
& \leq \sqrt{C \mathbf{D}\left(u_{o}\right)}\|\varphi\|_{L^{2}(B \times(0, \infty))},
\end{aligned}
$$

where we used the second term of the uniform bound (8.8) from above. But this implies the existence of the weak time derivative $\partial_{t} u$ of $u$ in $L^{2}$, that is, $\partial_{t} u \in$ $L^{2}\left(B \times(0, \infty), \mathbb{R}^{3}\right)$, and therefore $u \in W^{1,2}\left(B \times(0, \infty), \mathbb{R}^{3}\right)$ with

$$
\int_{0}^{\infty} \int_{B}\left|\partial_{t} u\right|^{2} d x d t \leq C \mathbf{D}\left(u_{o}\right)
$$

Moreover, we have

$$
\Delta_{t}^{h_{i}} u_{h_{i}} \rightarrow \partial_{t} u \quad \text { weakly in } L^{2}\left(B \times(0, \infty), \mathbb{R}^{3}\right) .
$$

Next, from (8.9) we conclude that for $0 \leq t_{1}<t_{2}$ there holds

$$
\sup _{i \in \mathbb{N}} \int_{t_{1}}^{t_{2}} \int_{B}\left|D u_{h_{i}}\right|^{2} d x d t \leq C \mathbf{D}\left(u_{o}\right)\left(t_{2}-t_{1}\right)
$$

and

$$
\sup _{i \in \mathbb{N}} \int_{t_{1}}^{t_{2}} \int_{B}\left|\Delta_{t}^{h_{i}} u_{h_{i}}\right|^{2} d x d t \leq 2 C \mathbf{D}\left(u_{o}\right) .
$$

By Fatou's Lemma we therefore have

$$
\begin{aligned}
\int_{t_{1}}^{t_{2}} \liminf _{i \rightarrow \infty} & \int_{B}\left|D u_{h_{i}}\right|^{2}+\left|\Delta_{t}^{h_{i}} u_{h_{i}}\right|^{2} d x d t \\
& \leq \liminf _{i \rightarrow \infty} \int_{t_{1}}^{t_{2}} \int_{B}\left|D u_{h_{i}}\right|^{2}+\left|\Delta_{t}^{h_{i}} u_{h_{i}}\right|^{2} d x d t \\
& \leq C \mathbf{D}\left(u_{o}\right)\left(2+t_{2}-t_{1}\right)<\infty
\end{aligned}
$$

and thus for almost every $t \in\left(t_{1}, t_{2}\right)$ we have

$$
\liminf _{i \rightarrow \infty} \int_{B}\left|D u_{h_{i}}(\cdot, t)\right|^{2}+\left|\Delta_{t}^{h_{i}} u_{h_{i}}(\cdot, t)\right|^{2} d x<\infty .
$$

Hence, for fixed $t \in\left(t_{1}, t_{2}\right)$ satisfying (8.16) ) and a non-relabeled subsequence (which may depend on $t$ ), we have

$$
\sup _{i \in \mathbb{N}} \int_{B}\left|D u_{h_{i}}(\cdot, t)\right|^{2}+\left|\Delta_{t}^{h_{i}} u_{h_{i}}(\cdot, t)\right|^{2} d x<\infty .
$$

Next, we consider $k_{i} \in \mathbb{N}$ such that $k_{i} h_{i} \leq t<\left(k_{i}+1\right) h_{i}$. Then $u_{h_{i}}(x, t)=u_{k_{i}, h_{i}}(x)$ is a minimizer of the functional

$$
\mathbf{F}_{k_{i}, h_{i}}(\tilde{u})=\mathbf{D}(\tilde{u})+2 \mathbf{V}_{H}\left(\tilde{u}, u_{o}\right)+\frac{1}{2 h_{i}} \int_{B}\left|\tilde{u}-u_{k_{i}-1, h_{i}}\right|^{2} d x
$$


in the class $\mathcal{S}\left(u_{o} ; A, \sigma\right)$. Moreover, $u_{k_{i}, h_{i}}$ solves the Euler-Lagrange system associated to $\mathbf{F}_{k_{i}, h_{i}}$; that is,

$$
\frac{u_{k_{i}, h_{i}}-u_{k_{i}-1, h_{i}}}{h_{i}}-\Delta u_{k_{i}, h_{i}}+2\left(H \circ u_{k_{i}, h_{i}}\right) D_{1} u_{k_{i}, h_{i}} \times D_{2} u_{k_{i}, h_{i}}=0
$$

weakly on $B$. We note that

$$
\frac{u_{k_{i}, h_{i}}-u_{k_{i}-1, h_{i}}}{h_{i}}=\Delta_{t}^{h_{i}} u_{h_{i}}(\cdot, t) .
$$

Since $\Delta_{t}^{h_{i}} u_{h_{i}}(\cdot, t)$ is a bounded sequence in $L^{2}\left(B, \mathbb{R}^{3}\right)$, we can again pass to another subsequence such that

$$
\Delta_{t}^{h_{i}} u_{h_{i}}(\cdot, t)=\frac{u_{k_{i}, h_{i}}-u_{k_{i}-1, h_{i}}}{h_{i}} \rightarrow-f(\cdot, t) \quad \text { weakly in } L^{2}\left(B, \mathbb{R}^{3}\right) .
$$

Therefore, all assumptions of Lemma 7.5 (i) are fulfilled, and we conclude that the weak limit $u(\cdot, t)$ satisfies the limit system, that is,

$$
-\Delta u(\cdot, t)+2(H \circ u(\cdot, t)) D_{1} u(\cdot, t) \times D_{2} u(\cdot, t)=f(\cdot, t)
$$

weakly on $B$. We note that this holds whenever $t \in\left(t_{1}, t_{2}\right)$ is chosen such that (8.16) holds. Since the subsequence may depend on $t$, this is not enough to identify $-f(\cdot, t)$ as $\partial_{t} u(\cdot, t)$ and to guarantee that $u$ is the desired weak solution of (1.1).

For $a>0$ and $i \in \mathbb{N}$ we define

$$
\Lambda_{i, a}:=\left\{t \in\left(t_{1}, t_{2}\right): \int_{B}\left|D u_{h_{i}}(\cdot, t)\right|^{2}+\left|\Delta_{t}^{h_{i}} u_{h_{i}}(\cdot, t)\right|^{2} d x>a\right\} .
$$

Then, for the measure $\left|\Lambda_{i, a}\right|$ we deduce from (8.14) and (8.15) that

$$
a\left|\Lambda_{i, a}\right| \leq \int_{t_{1}}^{t_{2}} \int_{B}\left|D u_{h_{i}}(\cdot, t)\right|^{2}+\left|\Delta_{t}^{h_{i}} u_{h_{i}}(\cdot, t)\right|^{2} d x d t \leq C \mathbf{D}\left(u_{o}\right)\left(2+t_{2}-t_{1}\right)
$$

so that

$$
\left|\Lambda_{i, a}\right| \leq \frac{C \mathbf{D}\left(u_{o}\right)\left(2+t_{2}-t_{1}\right)}{a} .
$$

We now define modified sequences $\left(\tilde{u}_{h_{i}}\right)_{i \in \mathbb{N}}$ and $\left(\tilde{f}_{h_{i}}\right)_{i \in \mathbb{N}}$ according to

$$
\tilde{u}_{h_{i}}(x, t):=\left\{\begin{array}{cl}
u(x, t) & \text { if } t \in \Lambda_{i, a}, \\
u_{h_{i}}(x, t) & \text { if } t \notin \Lambda_{i, a}
\end{array}\right.
$$

and

$$
\tilde{f}_{h_{i}}(x, t):=\left\{\begin{array}{cc}
f(x, t) & \text { if } t \in \Lambda_{i, a}, \\
-\Delta_{t}^{h_{i}} u_{h_{i}}(x, t) & \text { if } t \notin \Lambda_{i, a} .
\end{array}\right.
$$

This means, on the good slices $B \times\{t\}$ with $t \notin \Lambda_{i, a}$ we do not change $u_{h_{i}}(\cdot, t)$, while on the bad slices we take instead of $u_{h_{i}}(\cdot, t)$ the limit function $u(\cdot, t)$ and the associated right-hand side $f(\cdot, t)$ from the previous construction. We observe that for each fixed $a>0$ we still have $\tilde{u}_{h_{i}} \rightarrow u$ in $L^{\infty}\left(\left[t_{1}, t_{2}\right] ; L^{2}\left(B, \mathbb{R}^{3}\right)\right)$. Furthermore, for a.e. $t \in\left[t_{1}, t_{2}\right]$ we have

$$
-\Delta \tilde{u}_{h_{i}}(\cdot, t)+2\left(H \circ \tilde{u}_{h_{i}}(\cdot, t)\right) D_{1} \tilde{u}_{h_{i}}(\cdot, t) \times D_{2} \tilde{u}_{h_{i}}(\cdot, t)=\tilde{f}_{h_{i}}(\cdot, t),
$$

weakly on $B$. Now, if $t \notin \Lambda_{i, a}$, then we have

$$
\int_{B}\left|D \tilde{u}_{h_{i}}(\cdot, t)\right|^{2}+\left|\tilde{f}_{h_{i}}(\cdot, t)\right|^{2} d x=\int_{B}\left|D u_{h_{i}}(\cdot, t)\right|^{2}+\left|\Delta_{t}^{h_{i}} u_{h_{i}}(\cdot, t)\right|^{2} d x \leq a,
$$


while for a.e. $t \in \Lambda_{i, a}$, we have

$$
\int_{B}\left|D \tilde{u}_{h_{i}}(\cdot, t)\right|^{2}+\left|\tilde{f}_{h_{i}}(\cdot, t)\right|^{2} d x=\int_{B}|D u(\cdot, t)|^{2}+|f(\cdot, t)|^{2} d x<\infty .
$$

Combining the last and the second-to-last inequality we obtain that the sequences $\tilde{u}_{h_{i}}$ and $\tilde{f}_{h_{i}}$ satisfy the following inequality for a.e. $t \in\left[t_{1}, t_{2}\right]$ :

$$
\sup _{i \in \mathbb{N}} \int_{B}\left|D \tilde{u}_{h_{i}}(\cdot, t)\right|^{2}+\left|\tilde{f}_{h_{i}}(\cdot, t)\right|^{2} d x \leq \max \left\{a, \int_{B}|D u(\cdot, t)|^{2}+|f(\cdot, t)|^{2} d x\right\}<\infty .
$$

Next, we claim that the non-linear terms converge in the sense

$$
g(t):=\int_{B \times\{t\}} 2(H \circ u) D_{1} u \times D_{2} u \cdot \varphi d x=\lim _{i \rightarrow \infty} g_{i}(t),
$$

where

$$
g_{i}(t):=\int_{B \times\{t\}} 2\left(H \circ \tilde{u}_{h_{i}}\right) D_{1} \tilde{u}_{h_{i}} \times D_{2} \tilde{u}_{h_{i}} \cdot \varphi d x .
$$

In fact, for subsequences $\left\{i_{j}\right\}$ with $t \in \Lambda_{i_{j}, a}$ for all $j \in \mathbb{N}$, the convergence holds trivially, since then we have $\tilde{u}_{h_{i_{j}}}=u$. On the other hand, we can apply Lemma 7.5 on the time slices $B \times\{t\}$ with $t \in\left[t_{1}, t_{2}\right]$ for every subsequence $\left\{i_{j}\right\}$ with $t \notin \Lambda_{i_{j}, a}$ for all $j \in \mathbb{N}$, so that claim (8.18) follows from Lemma 7.5 (ii).

Next, we want to apply the dominated convergence theorem to the sequence $g_{i}:\left[t_{1}, t_{2}\right] \rightarrow \mathbb{R}_{+}$. We already know that $g_{i}(t) \rightarrow g(t)$ for a.e. $t \in\left[t_{1}, t_{2}\right]$. Further, we have

$$
\begin{aligned}
g_{i}(t) & \leq\|H\|_{L^{\infty}}\|\varphi\|_{L^{\infty}} \int_{B}\left|D \tilde{u}_{h_{i}}(\cdot, t)\right|^{2} d x \\
& \leq\|H\|_{L^{\infty}}\|\varphi\|_{L^{\infty}} \max \left\{a, \int_{B}|D u(\cdot, t)|^{2} d x\right\} .
\end{aligned}
$$

Since the right-hand side is in $L^{1}\left(\left[t_{1}, t_{2}\right], \mathbb{R}\right)$, we can apply the dominated convergence theorem to conclude that

$$
\begin{aligned}
\int_{t_{1}}^{t_{2}} \int_{B} 2 & (H \circ u) D_{1} u \times D_{2} u \cdot \varphi d x d t \\
& =\lim _{i \rightarrow \infty} \int_{t_{1}}^{t_{2}} \int_{B} 2\left(H \circ \tilde{u}_{h_{i}}\right) D_{1} \tilde{u}_{h_{i}} \times D_{2} \tilde{u}_{h_{i}} \cdot \varphi d x d t .
\end{aligned}
$$

On the right-hand side of the preceding equality we want to replace the sequence $\tilde{u}_{h_{i}}$ by the original sequence $u_{h_{i}}$. Taking into account the uniform bound (8.9) we can estimate the non-linear term $2\left(H \circ u_{h_{i}}\right) D_{1} u_{h_{i}} \times D_{2} u_{h_{i}} \cdot \varphi$ on the time slices $B \times\{t\}$ as follows:

$$
\begin{aligned}
& \int_{B \times\{t\}} \mid 2\left(H \circ u_{h_{i}}\right) D_{1} u_{h_{i}} \times D_{2} u_{h_{i}} \cdot \varphi \mid d x \\
& \leq\|H\|_{L^{\infty}}\|\varphi\|_{L^{\infty}} \int_{B}\left|D u_{h_{i}}(\cdot, t)\right|^{2} d x \leq C\|H\|_{L^{\infty}}\|\varphi\|_{L^{\infty}} \mathbf{D}\left(u_{o}\right) .
\end{aligned}
$$

We integrate this with respect to $t$ over $\Lambda_{i, a}$ and then use the measure estimate (8.17) to obtain

$$
\int_{\Lambda_{i, a}} \int_{B}\left|2\left(H \circ u_{h_{i}}\right) D_{1} u_{h_{i}} \times D_{2} u_{h_{i}} \cdot \varphi\right| d x d t \leq C\|H\|_{L^{\infty}}\|\varphi\|_{L^{\infty}} \mathbf{D}\left(u_{o}\right)\left|\Lambda_{i, a}\right|
$$




$$
\leq C\|H\|_{L^{\infty}}\|\varphi\|_{L^{\infty}} \mathbf{D}\left(u_{o}\right) \frac{\mathbf{D}\left(u_{o}\right)\left(2+t_{2}-t_{1}\right)}{a} .
$$

Similarly, since $\tilde{u}_{h_{i}}(\cdot, t) \equiv u(\cdot, t)$ for $t \in \Lambda_{i, a}$, we have

$$
\begin{aligned}
\int_{\Lambda_{i, a}} \int_{B}\left|2\left(H \circ \tilde{u}_{h_{i}}\right) D_{1} \tilde{u}_{h_{i}} \times D_{2} \tilde{u}_{h_{i}} \cdot \varphi\right| d x d t \\
\leq\|H\|_{L^{\infty}}\|\varphi\|_{L^{\infty}} \int_{\Lambda_{i, a}} \int_{B}|D u(\cdot, t)|^{2} d x d t .
\end{aligned}
$$

Joining the last two estimates we obtain

$$
\begin{gathered}
\left|\int_{t_{1}}^{t_{2}} \int_{B}\left[2\left(H \circ \tilde{u}_{h_{i}}\right) D_{1} \tilde{u}_{h_{i}} \times D_{2} \tilde{u}_{h_{i}}-2\left(H \circ u_{h_{i}}\right) D_{1} u_{h_{i}} \times D_{2} u_{h_{i}}\right] \cdot \varphi d x d t\right| \\
\quad=\left|\int_{\Lambda_{i, a}} \int_{B}[\ldots] d x d t\right| \leq \widetilde{C}\left[\frac{1}{a}+\int_{\Lambda_{i, a}} \int_{B}|D u(\cdot, t)|^{2} d x d t\right]
\end{gathered}
$$

for a constant $\widetilde{C}$ independent of $i$ and $a$. Now we let $a \rightarrow \infty$. In view of (8.19), we infer

$$
\begin{aligned}
\int_{t_{1}}^{t_{2}} \int_{B} 2(H \circ u) D_{1} u \times D_{2} u \cdot \varphi d x d t & \\
& =\lim _{i \rightarrow \infty} \int_{t_{1}}^{t_{2}} \int_{B} 2\left(H \circ u_{h_{i}}\right) D_{1} u_{h_{i}} \times D_{2} u_{h_{i}} \cdot \varphi d x d t
\end{aligned}
$$

whenever $\varphi \in C_{0}^{\infty}\left(B \times\left(t_{1}, t_{2}\right), \mathbb{R}^{3}\right)$. The last identity, the weak convergence $D u_{h_{i}} \rightarrow$ $D u$ in $L^{2}\left(B \times(0, \infty), \mathbb{R}^{3 \cdot 2}\right)$ and the weak convergence $\Delta_{t}^{h_{i}} u_{h_{i}} \rightarrow \partial_{t} u$ in $L^{2}(B \times$ $\left.(0, \infty), \mathbb{R}^{3}\right)$ from (8.13) allow us to pass to the limit $i \rightarrow \infty$ in (8.7), and this proves that $u$ solves the limit system. The above construction yields in particular that $u \in C^{0}\left([0, \infty) ; L^{2}\left(B, \mathbb{R}^{3}\right)\right) \cap L^{\infty}\left([0, \infty) ; W^{1,2}\left(B, \mathbb{R}^{3}\right)\right)$ and $\partial_{t} u \in L^{2}\left(B \times(0, \infty), \mathbb{R}^{3}\right)$. This proves Theorem 1.1

\section{Applichtions}

9.1. Existence of a variational flow. Here we give several sufficient conditions ensuring the existence of a weak solution to the Cauchy-Dirichlet problem (1.1). They follow from Theorem 1.1 and known criteria ensuring a spherical isoperimetric condition for the prescribed function $H$. For a detailed discussion of such criteria we refer to [30, 31, 6, 7].

Theorem 9.1. Let $A$ be convex and the closure of a $C^{2}$-domain in $\mathbb{R}^{3}$ and let the principal curvatures of $\partial A$ be bounded. By $\mathcal{H}_{\partial A}$ we denote the minimum of the principal curvatures of $\partial A$. Further, we consider initial and boundary data $u_{o} \in W^{1,2}(B, A)$ and $H \in L^{\infty}(A) \cap C^{0}(A)$. Then each of the conditions

$$
\begin{gathered}
\sup _{A}|H| \leq \sqrt{\frac{2 \pi}{3 \mathbf{D}\left(u_{o}\right)}}, \\
A \subseteq B_{R} \quad \text { and } \int_{\left\{\xi \in A:|H(\xi)| \geq \frac{3}{2 R}\right\}}|H|^{3} d x<\frac{9 \pi}{2}, \\
\sup _{A}|H|<\frac{3}{2} \sqrt[3]{\frac{4 \pi}{3 \mathcal{L}^{3}(A)}},
\end{gathered}
$$


$(9.4)$

for some $c<1$ suppose that $\mathcal{L}^{3}\{a \in A:|H(a)| \geq \tau\} \leq c \frac{4 \pi}{3} \tau^{-3}$ for any $\tau>0$, together with the curvature assumption

$$
|H(a)| \leq \mathcal{H}_{\partial A}(a) \quad \text { for } a \in \partial A,
$$

ensure the existence of a weak solution of (1.1) with the properties described in Theorem 1.1. The same conditions guarantee the sub-convergence of $u(\cdot, t)$ to a solution of the stationary $H$-surface system.

In the case $A \equiv \bar{B}_{R}(0) \subseteq \mathbb{R}^{3}$ the conditions (9.3) and (9.5) simplify to

$$
\sup _{B_{R}(0)}|H|<\frac{3}{2} \frac{1}{R}, \quad|H(a)| \leq \frac{1}{R} \quad \text { for } a \in \partial B_{R}(0) .
$$

Moreover, in this case we have that (9.2) is fulfilled, and thus both assumptions, that is (9.2) and (9.3), contain as special cases the preceding Hildebrandt-type assumptions and ensure the existence of a weak solution in the sense of Theorem 1.1 to the parabolic $H$-flow system (1.1).

9.2. Convergence to a stationary solution; proof of Theorem $\mathbf{1 . 2}$. Here we show that at least for a sequence $t_{j} \uparrow \infty$ the sequence $\left(u\left(\cdot, t_{j}\right)\right)_{j \in \mathbb{N}}$ converges to a solution $u_{\infty}$ of the Dirichlet problem to the stationary $H$-surface system, i.e.

$$
\left\{\begin{array}{c}
\Delta u_{\infty}=2\left(H \circ u_{\infty}\right) D_{1} u_{\infty} \times D_{2} u_{\infty} \quad \text { on } B, \\
u_{\infty}=u_{o} \quad \text { on } \partial B .
\end{array}\right.
$$

First of all, since $u \in L^{\infty}\left([0, \infty), W^{1,2}\left(B, \mathbb{R}^{3}\right)\right)$, we have

$$
\int_{B}|D u(\cdot, t)|^{2} d x \leq C<\infty
$$

for a.e. $t \in[0, \infty)$. Second, since $u$ solves the parabolic $H$-system weakly on $B \times(0, \infty)$ and since $\partial_{t} u \in L^{2}\left(B \times(0, \infty), \mathbb{R}^{3}\right)$, we have

$\int_{0}^{\infty} \int_{B}\left[\partial_{t} u(\cdot, t) \cdot \zeta+D u(\cdot, t) \cdot D \zeta+2(H \circ u(\cdot, t)) D_{1} u(\cdot, t) \times D_{2} u(\cdot, t) \cdot \zeta\right] d x \eta(t) d t=0$ whenever $\zeta \in L^{\infty}\left(B, \mathbb{R}^{3}\right) \cap W_{0}^{1,2}\left(B, \mathbb{R}^{3}\right)$ and $\eta \in C^{\infty}((0, \infty), \mathbb{R})$. But this implies, again for a.e. $t \in(0, \infty)$, that

$$
-\Delta u(\cdot, t)+2(H \circ u(\cdot, t)) D_{1} u(\cdot, t) \times D_{2} u(\cdot, t)=-\partial_{t} u(\cdot, t)
$$

weakly on $B$. Next, from the $L^{2}$-estimate for $\partial_{t} u$, i.e. from (8.12), we infer the existence of a sequence $T_{j} \rightarrow \infty$ such that for all $j \in \mathbb{N}$ we have

$$
\int_{T_{j}}^{T_{j}+1} \int_{B}\left|\partial_{t} u(\cdot, t)\right|^{2} d x \leq \frac{1}{2 j}
$$

and $T_{j}+1<T_{j+1}$. Defining

$$
\Gamma_{j}:=\left\{t \in\left[T_{j}, T_{j}+1\right]: \int_{B}\left|\partial_{t} u(\cdot, t)\right|^{2} d x d t \leq \frac{1}{j}\right\}
$$

we have $\left|\Gamma_{j}\right| \geq \frac{1}{2}$. By $E$ we denote the set of those $t \geq 0$ for which either (9.7) or (9.8) fail to hold. Then, we have $|E|=0$ and hence $\left|\widetilde{\Gamma}_{j}\right| \geq \frac{1}{2}$, where $\widetilde{\Gamma}_{j}:=\Gamma_{j} \backslash E$. 
From (8.14) and (8.15) we obtain

$$
\int_{\widetilde{\Gamma}_{j}} \int_{B}\left|D u_{h_{i}}(\cdot, t)\right|^{2}+\left|\Delta_{t}^{h_{i}} u_{h_{i}}(\cdot, t)\right|^{2} d x d t \leq C \mathbf{D}\left(u_{o}\right) .
$$

We now define

$$
M_{j, i}:=\left\{t \in \widetilde{\Gamma}_{j}: \int_{B}\left|D u_{h_{i}}(\cdot, t)\right|^{2}+\left|\Delta_{t}^{h_{i}} u_{h_{i}}(\cdot, t)\right|^{2} d x \leq 4 C \mathbf{D}\left(u_{o}\right)\right\} .
$$

Then we have $\left|M_{j, i}\right| \geq \frac{1}{4}$. Now, the Lemma of Eisen (cf. Lemma 2.4) implies that there exists a subsequence $M_{j, i_{\ell}}$ (possibly depending on $j$ ) such that

$$
\bigcap_{\ell \in \mathbb{N}} M_{j, i_{\ell}} \neq \emptyset \text {. }
$$

But this means that there exist $t_{j} \in\left[T_{j}, T_{j}+1\right]$ such that there holds

$$
\int_{B}\left|\partial_{t} u\left(\cdot, t_{j}\right)\right|^{2} d x \leq \frac{1}{j} \quad \forall j \in \mathbb{N}
$$

as well as

$$
\int_{B}\left|D u_{h_{i_{\ell}}}\left(\cdot, t_{j}\right)\right|^{2}+\left|\Delta_{t}^{h_{i_{\ell}}} u_{h_{i_{\ell}}}\left(\cdot, t_{j}\right)\right|^{2} d x \leq 4 C \mathbf{D}\left(u_{o}\right) \quad \forall j, \ell \in \mathbb{N} .
$$

Moreover, we claim that after passing to a subsequence of $\left\{i_{\ell}\right\}$, there holds

$$
\Delta_{t}^{h_{i_{\ell}}} u_{h_{i_{\ell}}}\left(\cdot, t_{j}\right) \rightarrow \partial_{t} u\left(\cdot, t_{j}\right) \quad \text { weakly in } L^{2}\left(B, \mathbb{R}^{3}\right) \text {, as } \ell \rightarrow \infty .
$$

In fact, by passing to a subsequence we can achieve weak convergence to a function $-\tilde{f} \in L^{2}\left(B, \mathbb{R}^{3}\right)$. Then, the convergence $u_{h_{i}} \rightarrow u$ in $C^{0}\left([0, T] ; L^{2}\left(B, \mathbb{R}^{3}\right)\right)$ and Lemma 7.5 imply that $u\left(\cdot, t_{j}\right)$ weakly solves the system (7.18) with $\tilde{f}$ on the righthand side, while by (9.8) and the construction of the sets $\widetilde{\Gamma}_{j}$, the same system is satisfied with $-\partial_{t} u\left(\cdot, t_{j}\right)$. This implies $-\tilde{f}=\partial_{t} u\left(\cdot, t_{j}\right)$ a.e. on $B$ and thereby the claim (9.10).

We recall that by construction, the sequence $t_{j} \uparrow \infty$ satisfies

$$
\lim _{j \rightarrow \infty} \int_{B}\left|\partial_{t} u\left(\cdot, t_{j}\right)\right|^{2} d x=0 .
$$

Moreover, we can achieve, after passing to a subsequence of $\{j\}$ if necessary, that

$$
u\left(\cdot, t_{j}\right) \rightarrow u_{\infty} \text { weakly in } W^{1,2}\left(B, \mathbb{R}^{3}\right) \text { and strongly in } L^{2}\left(B, \mathbb{R}^{3}\right),
$$

for some limit map $u_{\infty} \in W^{1,2}\left(B, \mathbb{R}^{3}\right)$. Next, we use the fact that the weak $L^{2}$ convergence is metrizable on the balls $\mathcal{B}_{R} \subset L^{2}\left(B, \mathbb{R}^{3}\right)$. This means that there is a metric $\mathbf{d}: \mathcal{B}_{R} \times \mathcal{B}_{R} \rightarrow \mathbb{R}_{+}$such that for functions $v_{k}, v \in \mathcal{B}_{R} \subset L^{2}\left(B, \mathbb{R}^{3}\right)$ we have

$$
v_{k} \rightarrow v \text { weakly in } L^{2}\left(B, \mathbb{R}^{3}\right) \quad \Longleftrightarrow \quad \mathbf{d}\left(v_{k}, v\right) \rightarrow 0
$$

as $k \rightarrow \infty$. We wish to apply this to the sequence $\Delta_{t}^{h_{i_{\ell}}} u_{h_{i_{\ell}}}$ as well as to $D u_{h_{i_{\ell}}}$, which satisfy the bound (9.9). We know from (9.10) that for a subsequence of $\left\{i_{\ell}\right\}$ that may depend on $t_{j}$, there holds $\Delta_{t}^{h_{i_{\ell}}} u_{h_{i_{\ell}}}\left(\cdot, t_{j}\right) \rightarrow \partial_{t} u\left(\cdot, t_{j}\right)$ weakly in $L^{2}\left(B, \mathbb{R}^{3}\right)$. Further, we can achieve $D u_{h_{i}}\left(\cdot, t_{j}\right) \rightarrow D u\left(\cdot, t_{j}\right)$ weakly in $L^{2}\left(B, \mathbb{R}^{3 \cdot 2}\right)$ as $\ell \rightarrow \infty$. Therefore, we may choose some $i_{j} \in \mathbb{N}$ such that

$$
\mathbf{d}\left(D u_{h_{i_{j}}}\left(\cdot, t_{j}\right), D u\left(\cdot, t_{j}\right)\right)+\mathbf{d}\left(\Delta_{t}^{h_{i_{j}}} u_{h_{i_{j}}}\left(\cdot, t_{j}\right), \partial_{t} u\left(\cdot, t_{j}\right)\right) \leq \frac{1}{j} .
$$


Since $u_{h_{i}} \rightarrow u$ in $C^{0}\left([0, T] ; L^{2}\left(B, \mathbb{R}^{3}\right)\right)$ as $i \rightarrow \infty$, we can furthermore assume

$$
\left\|u_{h_{i_{j}}}\left(\cdot, t_{j}\right)-u\left(\cdot, t_{j}\right)\right\|_{L^{2}\left(B, \mathbb{R}^{3}\right)} \leq \frac{1}{j} .
$$

Combining the last two convergences with (9.11) and (9.12), we deduce that

$$
\left\{\begin{array}{cc}
D u_{h_{i_{j}}}\left(\cdot, t_{j}\right) \rightarrow D u_{\infty} & \text { weakly in } L^{2}\left(B, \mathbb{R}^{3 \cdot 2}\right), \\
u_{h_{i_{j}}}\left(\cdot, t_{j}\right) \rightarrow u_{\infty} & \text { strongly in } L^{2}\left(B, \mathbb{R}^{3}\right), \\
\Delta_{t}^{h_{i_{j}}} u_{h_{i_{j}}}\left(\cdot, t_{j}\right) \rightarrow 0 & \text { weakly in } L^{2}\left(B, \mathbb{R}^{3}\right),
\end{array}\right.
$$

as $j \rightarrow \infty$. Therefore, all hypotheses of Lemma 7.5 are fulfilled for the sequence $\left(u_{h_{i_{j}}}\right)_{j \in \mathbb{N}}$, and the application yields that the corresponding non-linear $H$-terms converge in the limit; that is, we have

$$
\begin{aligned}
\int_{B} 2\left(H \circ u_{\infty}\right) D_{1} u_{\infty} \times D_{2} u_{\infty} \cdot \varphi d x \\
\quad=\lim _{j \rightarrow \infty} \int_{B} 2\left(H \circ u_{h_{i_{j}}}\left(\cdot, t_{j}\right)\right) D_{1} u_{h_{i_{j}}}\left(\cdot, t_{j}\right) \times D_{2} u_{h_{i_{j}}}\left(\cdot, t_{j}\right) \cdot \varphi d x
\end{aligned}
$$

for all $\varphi \in C_{0}^{\infty}\left(B, \mathbb{R}^{3}\right)$. From the construction of the maps $u_{h_{i_{j}}}\left(\cdot, t_{j}\right)$ we recall that on $B$, they weakly solve the system

$$
\Delta u_{h_{i_{j}}}\left(\cdot, t_{j}\right)=2\left(H \circ u_{h_{i_{j}}}\left(\cdot, t_{j}\right)\right) D_{1} u_{h_{i_{j}}}\left(\cdot, t_{j}\right) \times D_{2} u_{h_{i_{j}}}\left(\cdot, t_{j}\right)+\Delta_{t}^{h_{i_{j}}} u_{h_{i_{j}}}\left(\cdot, t_{j}\right) .
$$

But because of (9.13) and (9.14), we can pass to the limit $j \rightarrow \infty$ and conclude that $u_{\infty}$ solves the limit system, that is, $(9.6) 1$ on $B$. Further, since for each of the $u\left(\cdot, t_{j}\right)$ we have $u\left(\cdot, t_{j}\right)=u_{o}$ on $\partial B$ in the sense of traces, the limit map also attains the Dirichlet boundary values $u_{o}$ and is therefore a solution of the Dirichlet problem for the $H$-surface system with Dirichlet boundary values $u_{o}$.

At this stage we are almost done. By the result of Rivière [27, Theorem I.2] concerning the Heinz conjecture, we know that the limit map $u_{\infty}$ is continuous in $B$. For this it would suffice that $H$ is merely $L^{\infty}$. From Lemma 7.2 we then infer $u \in C_{\text {loc }}^{1, \alpha}\left(B, \mathbb{R}^{3}\right)$, and if the datum $u_{o}$ is a continuous function on $\partial B$, we can also conclude the continuity of $u_{\infty}$ up to the boundary, that is, we have $u_{\infty} \in C^{0}\left(\bar{B}, \mathbb{R}^{3}\right) \cap C_{\mathrm{loc}}^{1, \alpha}\left(B, \mathbb{R}^{3}\right)$ for any $\alpha \in(0,1)$. Assuming further that the prescribed function $H$ is itself locally Hölder continuous, then $D^{2} u_{\infty}$ is locally Hölder continuous on $B$ and $u_{\infty}$ solves the $H$-surface system in the classical sense on $B$; see [30, Theorem 5.3] for a brief summary as well as [24, 18, 34].

\section{RefERENCES}

[1] Y. Cai and S. Zhou, Existence and uniqueness of weak solutions for a non-uniformly parabolic equation. J. Funct. Anal. 257(10):3021-3042, 2009. MR2568684 (2011d:35226)

[2] Y. Chen and S. Levine, The existence of the heat flow of $H$-systems. Discrete Contin. Dyn. Syst. 8(1):219-236, 2002. MR 1877837 (2003c:35061)

[3] F. Duzaar, Variational inequalities and harmonic mappings. J. Reine Angew. Math. 374:2960, 1987. MR876220 (88m:58038)

[4] F. Duzaar and J. Grotowski, Existence of higher dimensional $H$-systems. Duke Mathematical J. 101(3):459-485, 2000. MR.1740684 (2000m:58026)

[5] F. Duzaar and G. Mingione. Second order parabolic systems, optimal regularity, and singular sets of solutions. Ann. Inst. Henri Poincaré Anal. Non Linéaire, 22:705-751, 2005. MR2172857(2008h:35139) 
[6] F. Duzaar and K. Steffen, Existence of hypersurfaces with prescibed mean curvature in Riemannian manifolds. Indiana Univ. Math. J. 45:1045-1093, 1996. MR.1444478 (98h:49049)

[7] F. Duzaar and K. Steffen, Parametric surfaces of least $H$-energy in a Riemannian manifold. Math. Ann. 314:197-244, 1999. MR.1697443 (2000h:58027)

[8] G. Eisen, A selection lemma for sequences of measurable sets, lower semicontinuity of multiple integrals. Manuscripta Math. 27:73-79, 1979. MR524978 (80h:28004)

[9] L. Evans and R. Gariepy, Measure theory and fine properties of functions. Studies in Advanced Mathematics. CRC Press, Boca Raton, FL, 1992. MR.1158660 (93f:28001)

[10] H. Federer, Geometric Measure Theory. Springer, Berlin, 1969. MR0257325 (41:1976)

[11] A. Gastel and C. Scheven, Regularity of polyharmonic maps in the critical dimension. Comm. Anal. Geom. 17(2):185-226, 2009. MR2520907(2010i:58015)

[12] M. Giaquinta, Multiple Integrals in the Calculus of Variations and Nonlinear Elliptic Systems. Ann. Math. Studies 105, Princeton University Press, 1983. MR717034 (86b:49003)

[13] E. Giusti, Direct Methods in the Calculus of Variations. World Scientific Publishing Company, Tuck Link, Singapore, 2003. MR.1962933 (2004g:49003)

[14] R. Gulliver and J. Spruck, The Plateau problem for surfaces of prescribed mean curvature in a cylinder. Invent. Math. 13:169-178, 1971. MR0298515(45:7567)

[15] R. Gulliver and J. Spruck, Existence theorems for parametric surfaces of prescribed mean curvature. Indiana Univ. Math. J. 22:445-472, 1972. MR0308904 (46:8016)

[16] J. Haga, K. Hoshino and N. Kikuchi, Construction of harmonic map flows through the method of discrete Morse flows. Comput. Vis. Sci. 7:53-59, 2004. MR2065958 (2005c:53086)

[17] E. Heinz, Über die Existenz einer Fläche konstanter mittlerer Krümmung mit gegebener Berandung. Math. Ann. 137:258-287, 1954. MR0070013 (16:1115b)

[18] E. Heinz and F. Tomi, Zu einem Satz von Hildebrandt über das Randverhalten von Minimalflächen. Math. Z. 111:372-386, 1969. MR0266066 (42:975)

[19] S. Hildebrandt, Randwertprobleme für Flächen mit vorgeschriebener mittlerer Krümmung und Anwendungen auf die Kapillaritätstheorie I. Math. Z. 112:205-213, 1969. MR0250208 $(40: 3448)$

[20] S. Hildebrandt, Einige Bemerkungen über Flächen vorgeschriebener mittlerer Krümmung. Math. Z. 115:169-178, 1970. MR0266115 (42:1024)

[21] S. Hildebrandt and H. Kaul, Two-dimensional variational problems with obstructions, and Plateau's problem for $H$-surfaces in a Riemannian manifold. Comm. Pure Appl. Math. 25:187-223, 1972. MR0296829 (45:5888)

[22] N. Kikuchi, An approach to the construction of Morse flows for variational functionals. $\mathrm{Ne}$ matics (Orsay 1990), NATO Adv. Sci. Inst. Ser. C Math. Phys. Sci., Kluwer Acad. Publ., Dordrecht. 332:195-199, 1991. MR1178095 (93i:58041)

[23] C. Leone, M. Misawa and A. Verde, A global existence result for the heat flow of higher dimensional H-systems. J. Math. Pures Appl. (9) 97:282-294, 2012. MR2887626

[24] C. B. Morrey, Multiple integrals in the Calculus of Variations. Berlin, Heidelberg, New York, Springer-Verlag 1966. MR0202511 (34:2380)

[25] R. Moser, Weak solutions of a biharmonic map heat flow. Adv. Calc. Var. 2:73-92, 2009. MR2494507 (2010a:58022)

[26] O. Rey, Heat flow for the equation of surfaces with prescribed mean curvature. Math. Ann. 293:123-146, 1991. MR1125012 (92k:58263)

[27] T. Rivière, Conservation laws for conformally invariant variational problems. Invent. Math. 168,1-22, 2007. MR2285745 (2008d:58010)

[28] J. Simon, Compact sets in the space $L^{p}(0, T ; B)$. Ann. Mat. Pura Appl. (4) 146:65-96, 1987. MR.916688 (89c:46055)

[29] L. Simon, Lectures on Geometric Measure Theory. Proc. of Centre for Math. Anal. 3, Australian National Univ., 1983. MR756417 (87a:49001)

[30] K. Steffen, Isoperimetric inequalities and the problem of Plateau. Math. Ann. 222:97-144, 1976. MR0417903 (54:5951)

[31] K. Steffen, On the existence of surfaces with prescribed mean curvature and boundary. Math. Z. 146:113-135, 1976. MR0394394(52:15196)

[32] M. Struwe, On the evolution of harmonic mappings of Riemannian surfaces. Comm. Math. Helv. 60:558-581, 1985. MR826871 (87e:58056)

[33] M. Struwe, The existence of surfaces of constant mean curvature with free boundaries. Acta Math. 160:19-64, 1988. MR926524(89a:53012) 
[34] F. Tomi, Ein einfacher Beweis eines Regularitätssatzes für schwache Lösungen gewisser elliptischer Systeme. Math. Z. 112:214-218, 1969. MR0257549(41:2199)

[35] H. Wente, An existence theorem for surfaces of constant mean curvature. J. Math. Appl. 26:318-344, 1969. MR0243467 (39:4788)

Department Mathematik, Universität Erlangen-Nürnberg, Cauerstrasse 11, 91058 ERlangen, Germany

E-mail address: boegelein@math.fau.de

Department Mathematik, Universität Erlangen-Nürnberg, Cauerstrasse 11, 91058 ERlangen, Germany

E-mail address: duzaar@math.fau.de

Department Mathematik, Universität Erlangen-Nürnberg, Cauerstrasse 11, 91058 Erlangen, Germany

E-mail address: scheven@mi.uni-erlangen.de

Current address: Fakultät für Mathematik, Universität Duisburg-Essen, Forsthausweg 2, 47057 Duisburg, Germany

E-mail address: christoph.scheven@uni-due.de 